Supporting Information for

\title{
Visible-Light-Induced Regioselective Dicarbonylation of Indolizines with Oxoaldehydes via Direct C-H Functionalization
}

\author{
Lili Teng, Xiang Liu, Pengfeng Guo, Yue Yu*, and Hua Cao* \\ School of Chemistry and Chemical Engineering and Guangdong Cosmetics Engineering \& Technology Research \\ Center, Guangdong Pharmaceutical University, Zhongshan 528458, P.R. of China \\ E-mail: yuyue@gdpu.edu.cn.; caohua@gdpu.edu.cn
}

Table of Contents:

1. General considerations

2. Experimental procedures and characterization data

2.1 Experimental procedures

2.2 Characterization data

3. NMR spectra for new compounds

4. References

S46 


\section{General considerations}

Analytical thin layer chromatography was carried out using silica gel GF254, visualized under UV light (at $254 \mathrm{~nm})$. Proton NMR $\left({ }^{1} \mathrm{H}\right)$ were recorded at $400 \mathrm{MHz}$, and Carbon $\mathrm{NMR}\left({ }^{13} \mathrm{C}\right)$ at $100 \mathrm{MHz} \mathrm{NMR}$ spectrometer. ${ }^{1} \mathrm{H}$ and ${ }^{13} \mathrm{C}$ NMR spectra were recorded using a Bruker DRX-400 spectrometer using $\mathrm{CDCl}_{3}$ as solvent. The chemical shifts are referenced to signals at 7.26 and $77.0 \mathrm{ppm}$, respectively. Multiplicities are abbreviated as: s, singlet; $d$, doublet; $t$, triplet; $q$, quartet; $m$, multiplet. The data of HRMS was carried out on a high-resolution mass spectrometer (LCMS-IT-TOF). IR spectra were obtained either as potassium bromide pellets or as liquid films between two potassium bromide pellets with a Bruker TENSOR 27 spectrometer. All the heat sources used in the experiment are heating mantle. Analytical thin layer chromatography was performed on $0.20 \mathrm{~mm}$ silica gel HSGF-254 plates (Huanghai, China), Column chromatography was performed on 200-300 mesh silica gel (Huanghai, China).

Photochemical reaction experiment were carried out on a PL-SX100A Model Multi-channel photochemical reaction instrument ( the light source is $20 \mathrm{~W}$ blue LED, the working current is $0.5-1.7 \mathrm{~A}$, the input power is $120 \mathrm{~W}$, the temperature is controlled by circulating water cooling, and the stirring speed is $0-1500 \mathrm{r} / \mathrm{min}$ ). UV-vis absorption spectra were recorded on a Shimadzu UV-2600 spectrophotometer. Fluorescence data measurements were carried out on a Thermo Scientific Lumina Model Fluorescence spectrometer (Power Maximum: $240 \mathrm{~W}$, Frequency Range: 50/60 Hz, AC Voltage: 100-240 V). 


\section{Experimental procedures and characterization data}

\subsection{Experimental procedures}

Synthesis of substrate 1 according to the following procedure:

The substrates 1a-1s are known and were prepared according to the procedures in the literature. ${ }^{1}$ As exemplified for 1a:

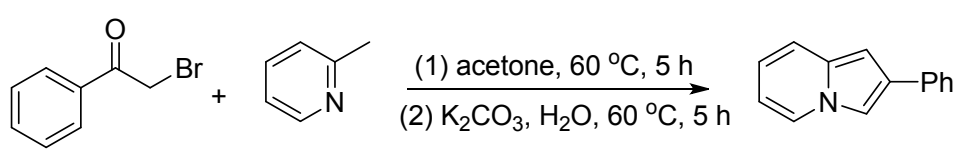

A solution of 2-picoline ( $0.93 \mathrm{~g}, 10 \mathrm{mmol}, 1.0$ equiv.) and 2 bromoacetophenone (1.99 g, $10 \mathrm{mmol}, 1.0$ equiv) in acetone $(50 \mathrm{~mL})$ were added to a $100 \mathrm{~mL}$ round bottom flask and heated with a heating mantle for 5 hours to $60{ }^{\circ} \mathrm{C}$. The precipitate obtained by filtration separation was redissolved in $20 \mathrm{~mL}$ of hot water $\left(60{ }^{\circ} \mathrm{C}\right)$. Then, $\mathrm{K}_{2} \mathrm{CO}_{3}\left(1.38 \mathrm{~g}, 10 \mathrm{mmol}, 1.0\right.$ equiv) was added and heated at $60{ }^{\circ} \mathrm{C}$ for 5 hours. After filtration and drying in vacuo, a white solid compound 1a was obtained in $50 \%$ overall yield ( $965 \mathrm{mg}, 5 \mathrm{mmol}$ ) without further purification.

The substrates 1t-1v are known and were prepared according to the procedures in the literature. ${ }^{2}$ As exemplified for $1 \mathbf{u}$ :

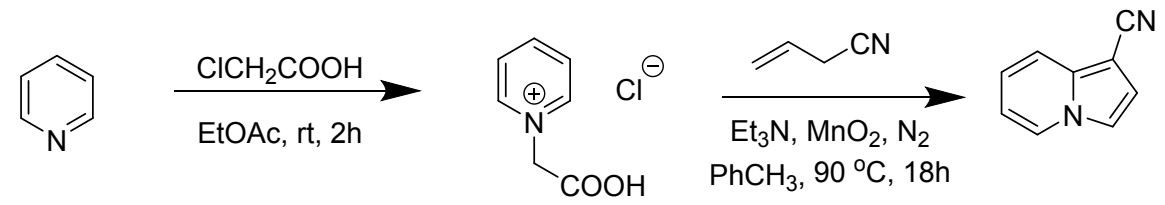

A mixture of pyridine $(8.10 \mathrm{~mL}, 100 \mathrm{mmol})$ and chloroacetic acid $(9.45 \mathrm{~g}, 100 \mathrm{mmol})$ in EtOAc $(20 \mathrm{~mL})$ was stirred at reflux for $2 \mathrm{~h}$. After cooling to ambient temperature the product was filtered off, washed with EtOAc, and dried; yield $16.95 \mathrm{~g}(98 \%)$, colorless solid. A mixture of $\mathrm{N}$-(carboxymethyl) pyridinium chloride (692 $\mathrm{mg}, 4.00 \mathrm{mmol})$, the required unsaturated nitrile $(20 \mathrm{~mol})$, triethylamine $(0.66 \mathrm{~mL}, 4.8 \mathrm{mmol})$ and $\mathrm{MnO}_{2}$ $(2.78 \mathrm{~g}, 23 \mathrm{mmol})$ in toluene $(40 \mathrm{~mL})$ was stirred under $\mathrm{N}_{2}$ at $90{ }^{\circ} \mathrm{C}$ with a heating mantle for $18 \mathrm{~h}$. After cooling to ambient temperature, the mixture was filtered through Celite, and the Celite was washed with acetone. The combined filtrates were evaporated in vacuo and the product isolated by flash chromatography on silica gel eluting with EtOAc-hexane (1:29) followed by EtOAc-hexane (1:9).

\section{Synthesis of substrates 2 according to the following procedure:}

The substrates $2 \mathbf{a}-\mathbf{2 g}$ are known and were prepared according to the procedures in the literature. ${ }^{3}$ 


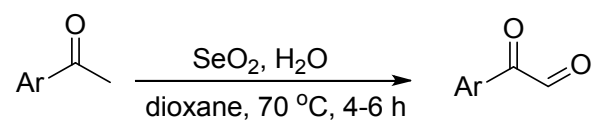

As exemplified for 2-oxo-2-phenylacetaldehyde 2a: Water $(2.5 \mathrm{~mL})$ was added to a stirred solution of $\mathrm{SeO}_{2}$ $(16.64 \mathrm{~g}, 150 \mathrm{mmol})$ in dioxane $(40 \mathrm{~mL})$ and heated to reflux with a heating mantle until the $\mathrm{SeO}_{2}$ dissolved. Acetophenone $(10.0 \mathrm{~g}, 83.3 \mathrm{mmol})$ was added to the solution and the reaction was maintained at reflux temperatures. After the reaction was complete, as monitored by TLC, the mixture was filtered through a filter paper and then filtered through a pad of Celite. The filtrate was evaporated to afford a crude product that was purified by distillation under reduced pressure. The fraction separated at $95-97{ }^{\circ} \mathrm{C} / 25$ Torr yielded the product as a yellow liquid. This liquid was dissolved in hot water $(40 \mathrm{~mL})$ and allowed to crystallize to afford 2a as a white solid $(10.39 \mathrm{~g}, 82 \%)$.

\section{Synthesis of product 3 according to the following procedure:}

As exemplified for 3a: A $25 \mathrm{~mL}$ sealed tube was charged with a stirring bar, and 2-phenylindolizine 1a $(0.097 \mathrm{~g}, 1.0$ equiv), 2-oxo-2-phenylacetaldehyde 2a ( $0.134 \mathrm{~g}, 2$ equiv), rose bengal $(0.015 \mathrm{~g}, 0.03$ equiv), DMSO $(3 \mathrm{~mL})$ were added. The reaction was irradiated with a $20 \mathrm{~W}$ blue LED at room temperature stirring for $12 \mathrm{~h}$ and monitored by TLC. The reaction mixture was then diluted with EtOAc and water, extracted with EtOAc. The extract was dried with $\mathrm{Na}_{2} \mathrm{SO}_{4}$. The solvent was removed with a rotary evaporator. The residue was purified by flash column chromatography (eluent: $\mathrm{PE} / \mathrm{EtOAc}=15 / 1, \mathrm{v} / \mathrm{v})$ to give product $3 \mathrm{a}(0.150 \mathrm{~g}$, $91 \%$ yield).

\section{Scale-up experiment:}

An oven-dried $50 \mathrm{~mL}$ Schlenk flask was charged with a stirring bar, and 2-phenylindolizine 1a (0.194 g, 1 mmol), 2-oxo-2-phenylacetaldehyde $2 \mathrm{a}(0.268 \mathrm{~g}, 2 \mathrm{mmol})$, catalyst rose bengal $(0.031 \mathrm{~g}, 3 \mathrm{~mol} \%)$, and DMSO $(15 \mathrm{~mL})$ were added. The reaction was irradiated with a $20 \mathrm{~W}$ blue LED at room temperature stirring for $24 \mathrm{~h}$ and monitored by TLC. The reaction mixture was then diluted with EtOAc and water, extracted with EtOAc. The combined organic extracts were dried over anhydrous $\mathrm{Na}_{2} \mathrm{SO}_{4}$, filtered, and concentrated. The solvent was removed with a rotary evaporator. The residue was purified by flash column chromatography (eluent: $\mathrm{PE} / \mathrm{EtOAc}=15 / 1, \mathrm{v} / \mathrm{v})$ to give product $\mathbf{3 a}(0.299 \mathrm{~g}, 91 \%$ yield $)$.

\section{Fluorescence experimental procedure:}


Weigh compound 3a of $0.0325 \mathrm{~g}$, add $1 \mathrm{~mL}$ DMSO into EP tube to dissolve, then transfer to $100 \mathrm{ml}$ volumetric bottle, continue to dilute with DMSO to scale, shake well, and set aside. Take $1 \mathrm{~mL}$ of the above solution, transfer it to a $10 \mathrm{~mL}$ volumetric bottle and dilute it to the scale, then mix it into $10^{-6} \mathrm{~mol} \cdot \mathrm{L}^{-1}$ standard reserve solution for fluorescence test. Turn on the power, turn on the fluorophotometer and the computer, preheat the instrument, initialize the instrument, and measure the fluorescence intensity according to the excitation and emission fluorescence spectrometry. Determine the fluorescence intensity of compound 3a in different solvents, replace DMSO with other solvents and repeat the above experimental steps.

\section{Determination of the Fluorescence Quantum Yield of compound 3a:}

The fluorescence quantum yield of the compound 3a was estimated by the relative method using Quinine sulfate dihydrate in sulfuric acid $\left(\mathrm{Ys}_{\mathrm{s}}=0.55\right)$ as the reference. compound 3a was dissolved in DMSO. The fluorescence quantum yield of compound 3a was calculated according to the following equation:

$$
Y u=Y s \times \frac{F u}{F \mathrm{~s}} \times \frac{A \mathrm{~s}}{A u} \quad(\mathrm{~A} \leq 0.05)
$$

The subscripts $\mathrm{u}$ and $\mathrm{s}$ represent the sample and the reference (Quinine sulfate dihydrate) molecule respectively. $\mathrm{Y}$ is the fluorescence quantum yield, $\mathrm{A}$ is the absorbance of molecules that were controlled below 0.05 for both molecules in the UV-vis absorption spectra, and F means the integrated emission area. According to the values obtained from fluorescence emission spectra, the fluorescence quantum yield of compound 3a is calculated to be 0.26 .

Extinction coefficient value was calculated for the compound 3a in DMSO using Lambert Beer law:A = عbc ; where $\mathrm{A}=$ Absorbance, $\varepsilon=$ extinction coefficient in $\mathrm{L} \mathrm{mol}^{-1} \mathrm{~cm}^{-1}, \mathrm{c}=$ concentration in $\mathrm{mol} \mathrm{L}^{-1} \& \mathrm{~b}=$ path length. When absorbance $\mathrm{A}=1.38$, path length $\mathrm{b}=1 \mathrm{~cm}$, substance concentration $\mathrm{c}=0.325 \times 10^{-3} \mathrm{~mol} \mathrm{~L}^{-1}$, the extinction coefficients was determined by the equation: $\mathrm{A}=\varepsilon b \mathrm{bc}, \varepsilon=4.23\left[\mathrm{~L} \cdot \mathrm{mol}^{-1} \mathrm{~cm}^{-1}\right] 10^{3}$.

\subsection{Characterization data}

\section{1-Phenyl-2-(2-phenylindolizin-3-yl)ethane-1,2-dione (3a)}<smiles>O=C(C(=O)c1c(-c2ccccc2)cc2ccccn12)c1ccccc1</smiles>

Flash column chromatography on silica gel (eluent: $\mathrm{PE} / \mathrm{EtOAc}=15 / 1, \mathrm{v} / \mathrm{v}$ ) to afford

3a. Yellow solid (147.9 mg, 91\%), mp 135-137 ${ }^{\circ} \mathrm{C}$; IR (KBr): 3039, 2915, 1583, 1419, 743, $699 \mathrm{~cm}^{-1} ;{ }^{1} \mathrm{H}$ NMR (400 MHz, $\left.\mathrm{CDCl}_{3}\right) \delta \mathrm{ppm} 10.09(\mathrm{~d}, \mathrm{~J}=6.9 \mathrm{~Hz}, 1 \mathrm{H})$, $7.67-7.62(\mathrm{~m}, 2 \mathrm{H}), 7.59(\mathrm{~d}, J=8.7 \mathrm{~Hz}, 1 \mathrm{H}), 7.50(\mathrm{t}, J=7.3 \mathrm{~Hz}, 1 \mathrm{H}), 7.33$ (q, $J=$ 
8.7, 8.0 Hz, 3H), $7.14(\mathrm{~d}, J=7.3 \mathrm{~Hz}, 1 \mathrm{H}), 7.07$ (t, $J=8.7 \mathrm{~Hz}, 3 \mathrm{H}), 7.00(\mathrm{t}, J=7.5 \mathrm{~Hz}, 2 \mathrm{H}), 6.53(\mathrm{~s}, 1 \mathrm{H}) .{ }^{13} \mathrm{C}$ NMR (100 MHz, $\left.\mathrm{CDCl}_{3}\right) \delta$ ppm 192.4, 183.2, 142.2, 139.4, 134.2, 134.0, 133.6, 130.4, 129.6, 129.6, 128.4, 128.0, 127.6, 126.6, 118.8, 118.5, 114.9, 106.1. HRMS MALDI $(\mathrm{m} / \mathrm{z})$ : calcd for $\mathrm{C}_{22} \mathrm{H}_{15} \mathrm{NO}_{2}[\mathrm{M}+\mathrm{Na}]^{+}$: 348.0995, found: 348.0996 .

\section{1-(8-Methyl-2-phenylindolizin-3-yl)-2-phenylethane-1,2-dione (3b)}

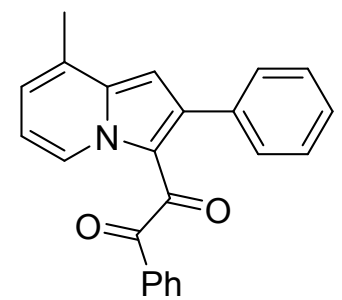

Flash column chromatography on silica gel (eluent: PE/EtOAc $=15 / 1, \mathrm{v} / \mathrm{v}$ ) to afford 3b. Green solid (140.7 mg, 83\%), mp 161-163 ${ }^{\circ} \mathrm{C}$; IR (KBr): 3067, 2929, 1571, 1405, 1240, 851, $757 \mathrm{~cm}^{-1} ;{ }^{1} \mathrm{H}$ NMR (400 MHz, $\left.\mathrm{CDCl}_{3}\right) \delta \mathrm{ppm} 9.96(\mathrm{~d}, J=6.8 \mathrm{~Hz}, 1 \mathrm{H})$, $7.64(\mathrm{~d}, J=7.2 \mathrm{~Hz}, 2 \mathrm{H}), 7.49(\mathrm{t}, J=7.4 \mathrm{~Hz}, 1 \mathrm{H}), 7.30(\mathrm{~d}, J=7.8 \mathrm{~Hz}, 2 \mathrm{H}), 7.17-$ $7.08(\mathrm{~m}, 4 \mathrm{H}), 7.00(\mathrm{t}, J=7.6 \mathrm{~Hz}, 2 \mathrm{H}), 6.96(\mathrm{t}, J=7.0 \mathrm{~Hz}, 1 \mathrm{H}), 6.53(\mathrm{~d}, J=0.7 \mathrm{~Hz}$, 1H), $2.50(\mathrm{~s}, 3 \mathrm{H}) .{ }^{13} \mathrm{C} \mathrm{NMR}\left(100 \mathrm{MHz}, \mathrm{CDCl}_{3}\right) \delta \mathrm{ppm} 192.5,183.2,141.7,139.8,134.3,134.0,133.6,130.4$, 129.5, 128.3, 127.9, 127.7, 127.5, 127.3, 126.0, 119.1, 114.9, 104.6, 18.1. HRMS MALDI (m/z): calcd for $\mathrm{C}_{23} \mathrm{H}_{17} \mathrm{NO}_{2}[\mathrm{M}+\mathrm{H}]^{+}: 340.1332$, found: 340.1338 .

\section{1-(7-Methyl-2-phenylindolizin-3-yl)-2-phenylethane-1,2-dione (3c)}

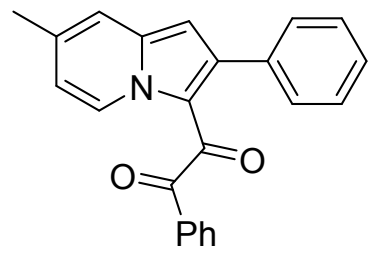

Flash column chromatography on silica gel (eluent: $\mathrm{PE} / \mathrm{EtOAc}=15 / 1, \mathrm{v} / \mathrm{v}$ ) to afford 3c. Green solid (147.5 mg, 87\%), mp 128-130 ${ }^{\circ} \mathrm{C}$; IR (KBr): 3054, 2973, 1584, 1419, 1236, 885, $645 \mathrm{~cm}^{-1} ;{ }^{1} \mathrm{H} \mathrm{NMR}\left(400 \mathrm{MHz}, \mathrm{CDCl}_{3}\right) \delta \mathrm{ppm} 9.97(\mathrm{~d}, J=$ $7.1 \mathrm{~Hz}, 1 \mathrm{H}), 7.64(\mathrm{~d}, J=7.0 \mathrm{~Hz}, 2 \mathrm{H}), 7.48(\mathrm{t}, J=7.4 \mathrm{~Hz}, 1 \mathrm{H}), 7.35-7.28(\mathrm{~m}$, 3H), $7.14(\mathrm{t}, J=7.3 \mathrm{~Hz}, 1 \mathrm{H}), 7.07(\mathrm{~d}, J=6.8 \mathrm{~Hz}, 2 \mathrm{H}), 6.99(\mathrm{t}, J=7.5 \mathrm{~Hz}, 2 \mathrm{H}), 6.87(\mathrm{~d}, J=6.9 \mathrm{~Hz}, 1 \mathrm{H}), 6.39$ (s, 1H), 2.44 (s, 3H). ${ }^{13} \mathrm{C}$ NMR (100 MHz, $\left.\mathrm{CDCl}_{3}\right) \delta \mathrm{ppm} \mathrm{192.6,} \mathrm{182.3,} \mathrm{142.5,} \mathrm{139.9,} \mathrm{138.1,} \mathrm{134.3,} \mathrm{134.1,}$ 133.5, 130.3 129.5, 129.0, 128.3, 127.9, 127.5, 118.4, 117.3, 117.2, 105.2, 21.5. HRMS MALDI (m/z): calcd for $\mathrm{C}_{23} \mathrm{H}_{17} \mathrm{NO}_{2}[\mathrm{M}+\mathrm{H}]^{+}:$340.1332, found: 340.1327 .

\section{1-(7-Methoxy-2-phenylindolizin-3-yl)-2-phenylethane-1,2-dione (3d)}<smiles>COc1ccn2c(C(=O)C(=O)c3ccccc3)c(-c3ccccc3)cc2c1</smiles>

Flash column chromatography on silica gel (eluent: $\mathrm{PE} / \mathrm{EtOAc}=15 / 1, \mathrm{v} / \mathrm{v}$ ) to afford 3d. Yellow solid (129.6 mg, 73\%), mp 144-145 ${ }^{\circ} \mathrm{C}$; IR (KBr): 3064, $2921,1564,1415,1226,858,747 \mathrm{~cm}^{-1} ;{ }^{1} \mathrm{H}$ NMR $\left(400 \mathrm{MHz}, \mathrm{CDCl}_{3}\right) \delta \mathrm{ppm}$ 
$9.97(\mathrm{~d}, J=7.5 \mathrm{~Hz}, 1 \mathrm{H}), 7.64(\mathrm{~d}, J=7.4 \mathrm{~Hz}, 2 \mathrm{H}), 7.48(\mathrm{t}, J=7.2 \mathrm{~Hz}, 1 \mathrm{H}), 7.32(\mathrm{~d}, J=7.4 \mathrm{~Hz}, 2 \mathrm{H}), 7.16-$ $7.12(\mathrm{~m}, 1 \mathrm{H}), 7.07$ (d, $J=7.2 \mathrm{~Hz}, 2 \mathrm{H}), 7.00$ (d, $J=7.3 \mathrm{~Hz}, 2 \mathrm{H}), 6.85(\mathrm{~s}, 1 \mathrm{H}), 6.73$ (d, $J=7.2 \mathrm{~Hz}, 1 \mathrm{H}), 6.34$ (s, 1H), $3.92(\mathrm{~s}, 3 \mathrm{H}) .{ }^{13} \mathrm{C} \mathrm{NMR}\left(100 \mathrm{MHz}, \mathrm{CDCl}_{3}\right) \delta \mathrm{ppm} 192.7,181.6,158.9,143.5,141.8,134.3,134.3,133.5$, 131.2, 130.3, 129.6, 128.3, 128.0, 127.6, 118.0, 108.4, 105.1, 96.6, 55.7. HRMS MALDI (m/z): calcd for $\mathrm{C}_{23} \mathrm{H}_{17} \mathrm{NO}_{3}[\mathrm{M}+\mathrm{Na}]^{+}:$378.1101, found: 378.1103 .

\section{1-Phenyl-2-(2-(p-tolyl)indolizin-3-yl)ethane-1,2-dione (3e)}

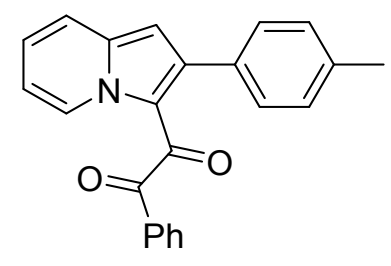

Flash column chromatography on silica gel (eluent: $\mathrm{PE} / \mathrm{EtOAc}=15 / 1, \mathrm{v} / \mathrm{v}$ ) to afford 3e. Green solid (130.6 mg, 77\%), mp 139-141 C; IR (KBr): 3075, 2921, $1679,1475,1246,861 \mathrm{~cm}^{-1} ;{ }^{1} \mathrm{H} \mathrm{NMR}\left(400 \mathrm{MHz}, \mathrm{CDCl}_{3}\right) \delta \mathrm{ppm} 10.07(\mathrm{~d}, J=6.8$ $\mathrm{Hz}, 1 \mathrm{H}), 7.64(\mathrm{~d}, J=7.2 \mathrm{~Hz}, 2 \mathrm{H}), 7.57(\mathrm{~d}, J=9.2 \mathrm{~Hz}, 1 \mathrm{H}), 7.50$ (t, $J=7.4 \mathrm{~Hz}$, 1H), $7.32(\mathrm{t}, J=7.8 \mathrm{~Hz}, 3 \mathrm{H}), 7.02(\mathrm{t}, J=6.9 \mathrm{~Hz}, 1 \mathrm{H}), 6.96(\mathrm{~d}, J=8.0 \mathrm{~Hz}, 2 \mathrm{H}), 6.80(\mathrm{~d}, J=7.6 \mathrm{~Hz}, 2 \mathrm{H}), 6.51$ (s, 1H), 2.24 (s, 3H). ${ }^{13} \mathrm{C}$ NMR (100 MHz, $\left.\mathrm{CDCl}_{3}\right) \delta \mathrm{ppm} \mathrm{192.5,} \mathrm{183.2,} \mathrm{142.3,} \mathrm{139.4,} \mathrm{137.8,} \mathrm{134.0,} \mathrm{133.6,}$ 131.1, 130.3, 129.5, 129.5, 128.3, 128.2, 126.6, 118.9, 118.4, 114.7, 106.0, 21.2. HRMS MALDI (m/z): calcd for $\mathrm{C}_{23} \mathrm{H}_{17} \mathrm{NO}_{2}[\mathrm{M}+\mathrm{Na}]^{+}: 362.1152$, found: 362.1153 .

\section{1-(2-(4-Ethylphenyl)indolizin-3-yl)-2-phenylethane-1,2-dione (3f)}

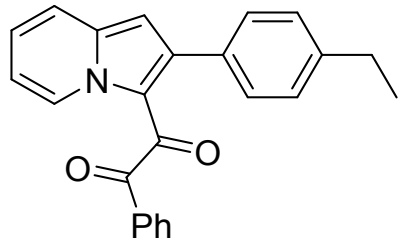

Flash column chromatography on silica gel (eluent: PE/EtOAc $=15 / 1, \mathrm{v} / \mathrm{v}$ ) to afford 3f. Green solid (125.4 mg, 71\%), mp 134-136 ${ }^{\circ} \mathrm{C}$; IR (KBr): 3068, 2917, 1682, 1584, 1261, $851 \mathrm{~cm}^{-1} ;{ }^{1} \mathrm{H}$ NMR (400 MHz, $\left.\mathrm{CDCl}_{3}\right) \delta \mathrm{ppm} 9.99(\mathrm{~s}, 1 \mathrm{H})$, $7.66(\mathrm{~d}, J=7.5 \mathrm{~Hz}, 2 \mathrm{H}), 7.55-7.48(\mathrm{~m}, 2 \mathrm{H}), 7.33(\mathrm{t}, J=7.7 \mathrm{~Hz}, 2 \mathrm{H}), 7.28(\mathrm{~s}$, 1H), 7.15 (t, $J=7.4 \mathrm{~Hz}, 1 \mathrm{H}), 7.09$ (d, $J=7.3 \mathrm{~Hz}, 2 \mathrm{H}), 7.01$ (t, $J=7.4 \mathrm{~Hz}, 2 \mathrm{H}), 6.49$ (s, 1H), 2.77 (q, $J=7.5$ $\mathrm{Hz}, 2 \mathrm{H}), 1.36(\mathrm{t}, J=7.5 \mathrm{~Hz}, 3 \mathrm{H}) .{ }^{13} \mathrm{C} \mathrm{NMR}\left(100 \mathrm{MHz}, \mathrm{CDCl}_{3}\right) \delta \mathrm{ppm} 192.5,182.9,141.8,138.4,134.4$, 134.1, 133.5, 131.2, 130.4, 129.6, 128.6, 128.3, 127.8, 127.5, 127.1, 118.7, 118.0, 105.9, 26.4, 15.4. HRMS MALDI (m/z): calcd for $\mathrm{C}_{24} \mathrm{H}_{19} \mathrm{NO}_{2}[\mathrm{M}+\mathrm{H}]^{+}: 354.1489$, found: 354.1485 .

\section{1-(2-(4-Bromophenyl)indolizin-3-yl)-2-phenylethane-1,2-dione (3g)}<smiles>O=C(C(=O)c1c(-c2ccc(Br)cc2)cc2ccccn12)c1ccccc1</smiles>

Flash column chromatography on silica gel (eluent: $\mathrm{PE} / \mathrm{EtOAc}=15 / 1, \mathrm{v} / \mathrm{v})$ to afford 3g. Green solid (165.2 mg, 82\%); mp 109-111 C; IR (KBr): 3061, 2920, 
1577, 1412, 1236, $865 \mathrm{~cm}^{-1} ;{ }^{1} \mathrm{H}$ NMR (400 MHz, $\left.\mathrm{CDCl}_{3}\right) \delta \mathrm{ppm} 10.06(\mathrm{~d}, J=6.9 \mathrm{~Hz}, 1 \mathrm{H}), 7.62(\mathrm{dd}, J=8.4$, $1.3 \mathrm{~Hz}, 2 \mathrm{H}), 7.58(\mathrm{~d}, J=8.8 \mathrm{~Hz}, 1 \mathrm{H}), 7.52(\mathrm{t}, J=7.4 \mathrm{~Hz}, 1 \mathrm{H}), 7.37-7.32(\mathrm{~m}, 3 \mathrm{H}), 7.10(\mathrm{~d}, J=8.3 \mathrm{~Hz}, 2 \mathrm{H})$, $7.07-7.03(\mathrm{~m}, 1 \mathrm{H}), 6.90(\mathrm{~d}, J=8.5 \mathrm{~Hz}, 2 \mathrm{H}), 6.49(\mathrm{~s}, 1 \mathrm{H}) .{ }^{13} \mathrm{C} \mathrm{NMR}\left(100 \mathrm{MHz}, \mathrm{CDCl}_{3}\right) \delta \mathrm{ppm} 192.7,183.0$, 140.6, 139.3, 134.0, 133.9, 133.0, 132.0, 130.6, 129.5, 129.5, 128.4, 126.8, 122.4, 118.6, 118.5, 115.1, 106.0. HRMS MALDI (m/z): calcd for $\mathrm{C}_{22} \mathrm{H}_{14} \mathrm{BrNO}_{2}[\mathrm{M}+\mathrm{H}]^{+}$: 404.0281, found: 404.0277.

\section{1-(2-(4-Fluorophenyl)indolizin-3-yl)-2-phenylethane-1,2-dione (3h)}

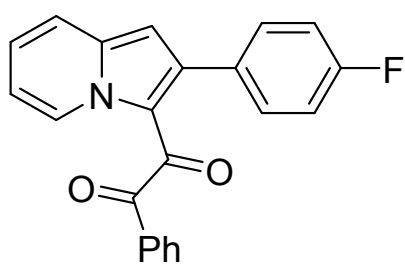

Flash column chromatography on silica gel (eluent: $\mathrm{PE} / \mathrm{EtOAc}=15 / 1, \mathrm{v} / \mathrm{v})$ to afford 3h. Green solid (152.7 mg, 89\%); mp 139-141 ${ }^{\circ} \mathrm{C}$; IR (KBr): 3063, 2951, 1672, 1577, 1246, 811, $689 \mathrm{~cm}^{-1} ;{ }^{1} \mathrm{H}$ NMR (400 MHz, $\left.\mathrm{CDCl}_{3}\right) \delta \mathrm{ppm}$ $10.06(\mathrm{~d}, J=6.9 \mathrm{~Hz}, 1 \mathrm{H}), 7.64(\mathrm{~d}, J=7.4 \mathrm{~Hz}, 2 \mathrm{H}), 7.57(\mathrm{~d}, J=9.0 \mathrm{~Hz}, 1 \mathrm{H})$, $7.50(\mathrm{t}, J=7.4 \mathrm{~Hz}, 1 \mathrm{H}), 7.32(\mathrm{t}, J=7.8 \mathrm{~Hz}, 3 \mathrm{H}), 7.05-6.99(\mathrm{~m}, 3 \mathrm{H}), 6.67(\mathrm{t}, J=8.7 \mathrm{~Hz}, 2 \mathrm{H}), 6.48(\mathrm{~s}, 1 \mathrm{H})$. ${ }^{13} \mathrm{C}$ NMR $\left(100 \mathrm{MHz}, \mathrm{CDCl}_{3}\right) \delta \mathrm{ppm} 192.6,183.0,162.6(J=248.2 \mathrm{~Hz}), 140.8,139.3,133.8,132.0(J=8.3$ Hz), $130.0(J=3.3 \mathrm{~Hz}), 129.4,128.4,126.7,118.8,118.5,115.0,114.3(J=21.7 \mathrm{~Hz}), 106.2$. HRMS MALDI (m/z): calcd for $\mathrm{C}_{22} \mathrm{H}_{14} \mathrm{FNO}_{2}[\mathrm{M}+\mathrm{H}]^{+}:$366.0901, found: 366.0901 .

\section{1-Phenyl-2-(2-(4-(trifluoromethyl)phenyl)indolizin-3-yl)ethane-1,2-dione (3i)}

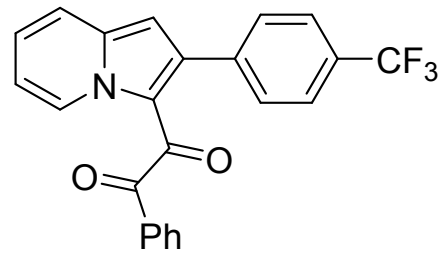

Flash column chromatography on silica gel (eluent: $\mathrm{PE} / \mathrm{EtOAc}=15 / 1, \mathrm{v} / \mathrm{v})$ to afford 3i. Yellow solid (157.2 mg, 80\%), mp 159-161 ${ }^{\circ} \mathrm{C}$; IR (KBr): 3067, $2921,1675,1422,1250,855,750 \mathrm{~cm}^{-1} ;{ }^{1} \mathrm{H} \mathrm{NMR}\left(400 \mathrm{MHz}, \mathrm{CDCl}_{3}\right) \delta \mathrm{ppm}$ $10.01(\mathrm{~d}, \mathrm{~J}=6.9 \mathrm{~Hz}, 1 \mathrm{H}), 7.55(\mathrm{~d}, \mathrm{~J}=8.8 \mathrm{~Hz}, 1 \mathrm{H}), 7.51(\mathrm{~d}, \mathrm{~J}=8.4 \mathrm{~Hz}, 2 \mathrm{H})$, $7.45(\mathrm{t}, \mathrm{J}=7.4 \mathrm{~Hz}, 1 \mathrm{H}), 7.33-7.28(\mathrm{~m}, 1 \mathrm{H}), 7.24(\mathrm{t}, \mathrm{J}=7.8 \mathrm{~Hz}, 2 \mathrm{H}), 7.17(\mathrm{~d}, \mathrm{~J}=8.0 \mathrm{~Hz}, 2 \mathrm{H}), 7.10(\mathrm{~d}, \mathrm{~J}=$

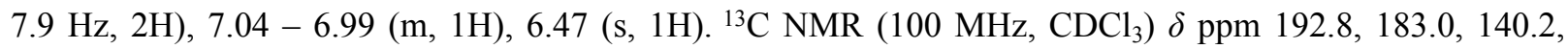
139.3, 137.9, 134.0, 133.8, 130.8, $129.8(J=32.6 \mathrm{~Hz}), 129.5,129.4,128.5,126.9,125.4,124.3(J=3.7 \mathrm{~Hz})$, 122.7 $(J=272.5 \mathrm{~Hz}), 118.6,115.3,106.1$. HRMS MALDI $(\mathrm{m} / \mathrm{z})$ : calcd for $\mathrm{C}_{23} \mathrm{H}_{14} \mathrm{~F}_{3} \mathrm{NO}_{2}[\mathrm{M}+\mathrm{H}]^{+}: 394.1049$, found: 394.1052 .

\section{1-(2-(4-(Benzyloxy)phenyl)indolizin-3-yl)-2-phenylethane-1,2-dione (3j)}




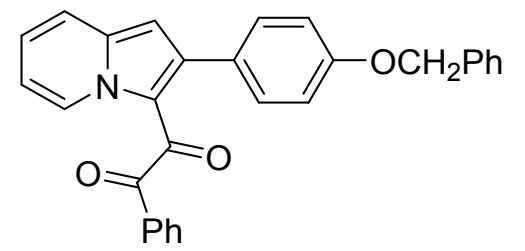

Flash column chromatography on silica gel (eluent: PE/EtOAc $=15 / 1$, v/v) to afford 3j. Green solid (157.4 mg, 73\%), mp 132-134 ${ }^{\circ} \mathrm{C}$; IR (KBr): 3039, 2952, 1682, 1408, 1241, 703, $695 \mathrm{~cm}^{-1} ;{ }^{1} \mathrm{H}$ NMR (400 MHz, $\left.\mathrm{CDCl}_{3}\right) \delta \mathrm{ppm} 10.08(\mathrm{~d}, J=7.1 \mathrm{~Hz}, 1 \mathrm{H}), 7.65(\mathrm{~d}, J=7.4 \mathrm{~Hz}, 2 \mathrm{H}), 7.57(\mathrm{~d}$, $J=8.7 \mathrm{~Hz}, 1 \mathrm{H}), 7.49(\mathrm{t}, J=7.4 \mathrm{~Hz}, 1 \mathrm{H}), 7.42(\mathrm{~d}, J=4.3 \mathrm{~Hz}, 4 \mathrm{H}), 7.36(\mathrm{~d}, J=4.4 \mathrm{~Hz}, 1 \mathrm{H}), 7.30(\mathrm{t}, J=7.8$ Hz, 3H), $7.04(\mathrm{~d}, J=6.4 \mathrm{~Hz}, 1 \mathrm{H}), 6.99(\mathrm{~d}, J=8.4 \mathrm{~Hz}, 2 \mathrm{H}), 6.62(\mathrm{~d}, J=8.6 \mathrm{~Hz}, 2 \mathrm{H}), 6.49$ (s, $1 \mathrm{H}), 4.98(\mathrm{~s}$, 2H). ${ }^{13} \mathrm{C}$ NMR (100 MHz, $\left.\mathrm{CDCl}_{3}\right) \delta \mathrm{ppm} 192.6,183.2,158.7,141.9,139.4,136.9,134.0,133.6,131.6,129.5$, $129.5,128.7,128.2,128.1,127.4,126.6,126.6,118.9,118.4,114.7,114.0,106.0,69.9$. HRMS MALDI (m/z): calcd for $\mathrm{C}_{29} \mathrm{H}_{21} \mathrm{NO}_{3}[\mathrm{M}+\mathrm{H}]^{+}: 432.1594$, found: 432.1590 .

\section{1-(2-(3-Chlorophenyl)indolizin-3-yl)-2-phenylethane-1,2-dione (3k)}

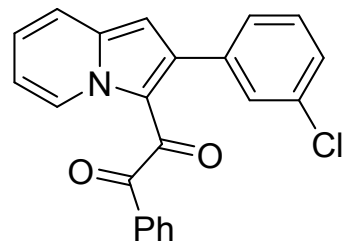

Flash column chromatography on silica gel (eluent: PE/EtOAc $=15 / 1, \mathrm{v} / \mathrm{v}$ ) to afford 3k. Green solid (120.3 mg, 67\%), mp 127-129 ${ }^{\circ} \mathrm{C}$; IR (KBr): 3051, 2920, $1685,1574,1246,861,716 \mathrm{~cm}^{-1} ;{ }^{1} \mathrm{H} \mathrm{NMR}\left(400 \mathrm{MHz}, \mathrm{CDCl}_{3}\right) \delta \mathrm{ppm} 10.00(\mathrm{~d}, J=$ $7.0 \mathrm{~Hz}, 1 \mathrm{H}), 7.63-7.60(\mathrm{~m}, 2 \mathrm{H}), 7.52(\mathrm{~d}, J=8.7 \mathrm{~Hz}, 1 \mathrm{H}), 7.46(\mathrm{t}, J=7.4 \mathrm{~Hz}, 1 \mathrm{H})$ $7.30(\mathrm{t}, J=7.8 \mathrm{~Hz}, 3 \mathrm{H}), 7.06(\mathrm{~d}, J=7.3 \mathrm{~Hz}, 1 \mathrm{H}), 6.99(\mathrm{~s}, 1 \mathrm{H}), 6.96-6.91(\mathrm{~m}, 3 \mathrm{H}), 6.44(\mathrm{~s}, 1 \mathrm{H}) .{ }^{13} \mathrm{C} \mathrm{NMR}$ $\left(100 \mathrm{MHz} \mathrm{CDCl}_{3}\right) \delta$ ppm 141.2, 140.2, 134.7, 133.6, 132.4, 132.1, 130.7, 129.6, 129.0, 128.1, 127.9, 126.4, 125.8, 125.6, 122.0, 121.9, 118.9, 112.7, 105.6, 94.6. HRMS MALDI (m/z): calcd for $\mathrm{C}_{22} \mathrm{H}_{14} \mathrm{ClNO}_{2}[\mathrm{M}+$ $\mathrm{H}]^{+}: 360.0786$, found: 360.0789 .

\section{1-(2-(3-Bromophenyl)indolizin-3-yl)-2-phenylethane-1,2-dione (3I)}

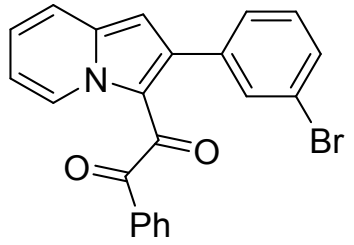

Flash column chromatography on silica gel (eluent: PE/EtOAc $=15 / 1, \mathrm{v} / \mathrm{v}$ ) to afford 31. Green solid (143.1 mg, 71\%), mp 150-152 ${ }^{\circ} \mathrm{C}$; IR (KBr): 3053, 2922 , $1675,1581,1236,865,731 \mathrm{~cm}^{-1} ;{ }^{1} \mathrm{H}$ NMR $\left(400 \mathrm{MHz}, \mathrm{CDCl}_{3}\right) \delta \mathrm{ppm} 10.07(\mathrm{~d}, J=$ $6.9 \mathrm{~Hz}, 1 \mathrm{H}), 7.71-7.67(\mathrm{~m}, 2 \mathrm{H}), 7.60(\mathrm{~d}, J=8.7 \mathrm{~Hz}, 1 \mathrm{H}), 7.54(\mathrm{t}, J=7.4 \mathrm{~Hz}, 1 \mathrm{H})$, $7.38(\mathrm{t}, J=7.8 \mathrm{~Hz}, 3 \mathrm{H}), 7.28(\mathrm{~d}, J=8.1 \mathrm{~Hz}, 1 \mathrm{H}), 7.15(\mathrm{t}, J=1.8 \mathrm{~Hz}, 1 \mathrm{H}), 7.08(\mathrm{t}, J=6.3 \mathrm{~Hz}, 2 \mathrm{H}), 6.95(\mathrm{t}, J=$ $7.8 \mathrm{~Hz}, 1 \mathrm{H}), 6.52(\mathrm{~s}, 1 \mathrm{H}) .{ }^{13} \mathrm{C} \mathrm{NMR}\left(100 \mathrm{MHz}, \mathrm{CDCl}_{3}\right) \delta \mathrm{ppm} 192.4,183.0,140.2,139.3,136.4,134.0,133.8$ 133.2, 131.0, 129.6, 129.5, 129.2, 129.0, 128.7, 126.8, 121.9, 118.6, 118.6, 115.2, 106.1. HRMS MALDI (m/z): calcd for $\mathrm{C}_{22} \mathrm{H}_{14} \mathrm{BrNO}_{2}[\mathrm{M}+\mathrm{H}]^{+}:$404.0281, found: 404.0277 . 


\section{1-(2-(3-Methoxyphenyl)indolizin-3-yl)-2-phenylethane-1,2-dione (3m)}

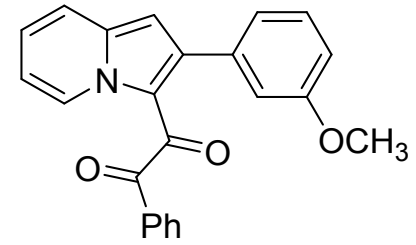

Flash column chromatography on silica gel (eluent: $\mathrm{PE} / \mathrm{EtOAc}=15 / 1, \mathrm{v} / \mathrm{v})$ to afford 3m. Yellow solid (138.5mg, 78\%), mp 129-131 ${ }^{\circ} \mathrm{C}$; IR (KBr): 3067, 2925, 1672, $1589,1241,714,652 \mathrm{~cm}^{-1} ;{ }^{1} \mathrm{H} \mathrm{NMR}\left(400 \mathrm{MHz}, \mathrm{CDCl}_{3}\right) \delta \mathrm{ppm}$ $10.08(\mathrm{~d}, J=7.2 \mathrm{~Hz}, 1 \mathrm{H}), 7.71-7.67(\mathrm{~m}, 2 \mathrm{H}), 7.59(\mathrm{~d}, J=8.8 \mathrm{~Hz}, 1 \mathrm{H}), 7.51(\mathrm{t}$, $J=7.4 \mathrm{~Hz}, 1 \mathrm{H}), 7.34(\mathrm{t}, J=7.7 \mathrm{~Hz}, 3 \mathrm{H}), 7.05(\mathrm{t}, J=7.0 \mathrm{~Hz}, 1 \mathrm{H}), 6.95(\mathrm{t}, J=7.9 \mathrm{~Hz}, 1 \mathrm{H}), 6.70(\mathrm{dd}, J=8.0$, $2.2 \mathrm{~Hz}, 2 \mathrm{H}), 6.55(\mathrm{~d}, J=11.7 \mathrm{~Hz}, 2 \mathrm{H}), 3.47(\mathrm{~s}, 3 \mathrm{H}) .{ }^{13} \mathrm{C} \mathrm{NMR}\left(100 \mathrm{MHz}, \mathrm{CDCl}_{3}\right) \delta \mathrm{ppm} \mathrm{192.2,} \mathrm{183.1,} \mathrm{158.7,}$ 142.0, 139.4, 135.6, 134.1, 133.6, 129.6, 129.6, 128.9, 128.4, 126.7, 122.9, 118.7, 118.5, 115.3, 114.9, 114.5, 105.9, 54.9. HRMS MALDI (m/z): calcd for $\mathrm{C}_{23} \mathrm{H}_{17} \mathrm{NO}_{3}[\mathrm{M}+\mathrm{H}]^{+}: 356.1281$, found: 356.1284 .

\section{1-(2-(2-Fluorophenyl)indolizin-3-yl)-2-phenylethane-1,2-dione (3n)}

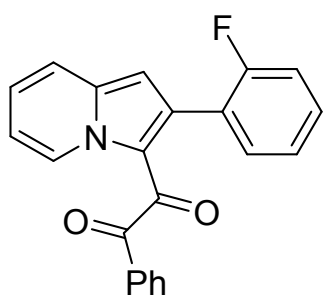

Flash column chromatography on silica gel (eluent: $\mathrm{PE} / \mathrm{EtOAc}=15 / 1, \mathrm{v} / \mathrm{v})$ to afford

3n. Green solid (130.4mg, 76\%), mp 150-152 ${ }^{\circ} \mathrm{C}$; IR (KBr): 3065, 2933, 1658, 1581, 1419, 1246, $760 \mathrm{~cm}^{-1} ;{ }^{1} \mathrm{H}$ NMR (400 MHz, $\left.\mathrm{CDCl}_{3}\right) \delta \mathrm{ppm} 10.06(\mathrm{~d}, J=7.0 \mathrm{~Hz}, 1 \mathrm{H})$, $7.62(\mathrm{dd}, J=14.9,8.2 \mathrm{~Hz}, 3 \mathrm{H}), 7.50(\mathrm{t}, J=7.5 \mathrm{~Hz}, 1 \mathrm{H}), 7.32(\mathrm{q}, J=8.7,8.1 \mathrm{~Hz}, 3 \mathrm{H})$, $7.13(\mathrm{dd}, J=13.6,5.5 \mathrm{~Hz}, 1 \mathrm{H}), 7.06(\mathrm{q}, J=7.0,6.4 \mathrm{~Hz}, 2 \mathrm{H}), 6.85(\mathrm{t}, J=7.4 \mathrm{~Hz}, 1 \mathrm{H}), 6.66(\mathrm{t}, J=9.0 \mathrm{~Hz}, 1 \mathrm{H})$, $6.56(\mathrm{~s}, 1 \mathrm{H}) .{ }^{13} \mathrm{C}$ NMR $\left(100 \mathrm{MHz}, \mathrm{CDCl}_{3}\right) \delta \mathrm{ppm} \mathrm{192.4,} \mathrm{183.0,} 159.8(J=247.4 \mathrm{~Hz}), 139.4,134.5,133.8$, 133.4, $132.8(J=2.6 \mathrm{~Hz}), 130.2(J=8.1 \mathrm{~Hz}), 129.6,129.4,128.3,126.5,123.3(J=3.7 \mathrm{~Hz}), 121.9(J=16.2$ $\mathrm{Hz}), 119.1,118.6,115.1,114.9(\mathrm{~J}=21.0 \mathrm{~Hz}), 106.8$. HRMS MALDI $(\mathrm{m} / \mathrm{z})$ : calcd for $\mathrm{C}_{22} \mathrm{H}_{14} \mathrm{FNO}_{2}[\mathrm{M}+\mathrm{Na}]^{+}$: 366.0901, found: 366.0898 .

\section{1-(2-(3,4-Dimethoxyphenyl)indolizin-3-yl)-2-phenylethane-1,2-dione (3o)}

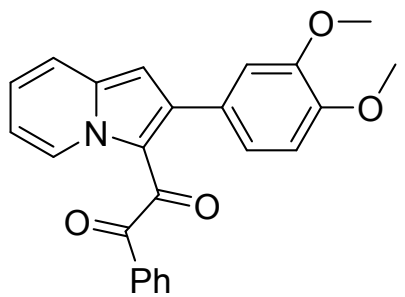

Flash column chromatography on silica gel (eluent: PE/EtOAc $=15 / 1, \mathrm{v} / \mathrm{v})$ to afford 3o. Yellow solid (157.9 mg, 82\%); mp 137-139 ${ }^{\circ} \mathrm{C}$; IR (KBr): 3052, 2924, 1588, 1405, 1230, $753 \mathrm{~cm}^{-1} ;{ }^{1} \mathrm{H}$ NMR $\left(400 \mathrm{MHz}, \mathrm{CDCl}_{3}\right) \delta \mathrm{ppm} 10.07(\mathrm{~d}$, $J=6.9 \mathrm{~Hz}, 1 \mathrm{H}), 7.66(\mathrm{~d}, J=7.6 \mathrm{~Hz}, 2 \mathrm{H}), 7.57(\mathrm{~d}, J=8.7 \mathrm{~Hz}, 1 \mathrm{H}), 7.48(\mathrm{~s}, 1 \mathrm{H})$, $7.32(\mathrm{t}, J=7.4 \mathrm{~Hz}, 3 \mathrm{H}), 7.03(\mathrm{~s}, 1 \mathrm{H}), 6.64(\mathrm{~d}, J=8.0 \mathrm{~Hz}, 1 \mathrm{H}), 6.52$ (d, $J=12.8 \mathrm{~Hz}, 3 \mathrm{H}), 3.80(\mathrm{~s}, 3 \mathrm{H}), 3.45(\mathrm{~s}$, 3H). ${ }^{13} \mathrm{C}$ NMR $\left(100 \mathrm{MHz}, \mathrm{CDCl}_{3}\right) \delta \mathrm{ppm} 192.4,183.0,148.9,147.8,142.0,139.4,134.1,133.6,129.5,129.4$, 128.3, 126.7, 126.6, 123.1, 118.9, 118.4, 114.8, 113.4, 110.5, 106.0, 55.9, 55.3. HRMS MALDI (m/z): calcd for $\mathrm{C}_{24} \mathrm{H}_{19} \mathrm{NO}_{4}[\mathrm{M}+\mathrm{H}]+:$ 386.1387, found: 386.1383 . 


\section{1-(2-(3,4-Dichlorophenyl)indolizin-3-yl)-2-phenylethane-1,2-dione (3p)}

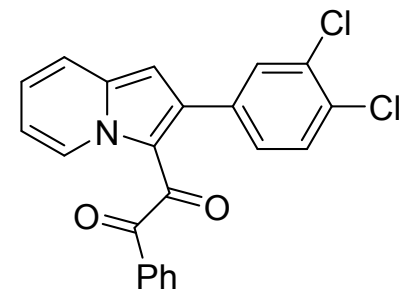

Flash column chromatography on silica gel (eluent: PE/EtOAc $=15 / 1, \mathrm{v} / \mathrm{v}$ ) to afford 3p. Green solid (143.5 mg, 73\%); mp 107-109 ${ }^{\circ} \mathrm{C}$; IR (KBr): 3057, 2912, 1473, 736, $694 \mathrm{~cm}^{-1} ;{ }^{1} \mathrm{H}$ NMR (400 MHz, $\left.\mathrm{CDCl}_{3}\right) \delta \mathrm{ppm} 10.06(\mathrm{~d}, J=6.9 \mathrm{~Hz}$, 1H), $7.70-7.65(\mathrm{~m}, 2 \mathrm{H}), 7.61(\mathrm{~d}, J=8.8 \mathrm{~Hz}, 1 \mathrm{H}), 7.55(\mathrm{t}, J=7.4 \mathrm{~Hz}, 1 \mathrm{H}), 7.38$ $(\mathrm{q}, J=7.8 \mathrm{~Hz}, 3 \mathrm{H}), 7.14(\mathrm{~d}, J=8.2 \mathrm{~Hz}, 1 \mathrm{H}), 7.11-7.04(\mathrm{~m}, 2 \mathrm{H}), 6.96(\mathrm{~d}, J=8.2 \mathrm{~Hz}, 1 \mathrm{H}), 6.51(\mathrm{~s}, 1 \mathrm{H}),{ }^{13} \mathrm{C}$ NMR (100 MHz, $\left.\mathrm{CDCl}_{3}\right) \delta$ ppm 192.6, 182.9, 139.2, 139.0, 134.2, 134.1, 133.6, 132.3, 132.1, 131.79, 129.6, $129.5,129.4,129.4,128.6,126.8,118.6,118.5,115.3,105.9$. HRMS MALDI $(\mathrm{m} / \mathrm{z})$ : calcd for $\mathrm{C}_{22} \mathrm{H}_{13} \mathrm{Cl}_{2} \mathrm{NO}_{2}$ $[\mathrm{M}+\mathrm{H}]^{+}: 394.0396$, found: 394.0400 .

\section{1-(2-(2,4-Dichlorophenyl)indolizin-3-yl)-2-phenylethane-1,2-dione (3q)}

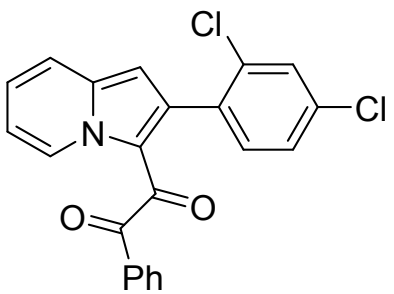

Flash column chromatography on silica gel (eluent: PE/EtOAc $=15 / 1, \mathrm{v} / \mathrm{v})$ to afford 3q. Green solid (141.5 mg, 72\%); mp 157-159 ${ }^{\circ} \mathrm{C}$; IR (KBr): 3067, 2919, 1571, 1402, 1246, $757 \mathrm{~cm}^{-1} ;{ }^{1} \mathrm{H}$ NMR $\left(400 \mathrm{MHz}, \mathrm{CDCl}_{3}\right) \delta \mathrm{ppm} 10.06(\mathrm{~d}, J=$ $6.8 \mathrm{~Hz}, 1 \mathrm{H}), 7.70-7.64(\mathrm{~m}, 2 \mathrm{H}), 7.60(\mathrm{~d}, J=8.8 \mathrm{~Hz}, 1 \mathrm{H}), 7.55(\mathrm{t}, J=7.4 \mathrm{~Hz}$, 1H), $7.41-7.34(\mathrm{~m}, 3 \mathrm{H}), 7.13(\mathrm{~d}, J=8.2 \mathrm{~Hz}, 1 \mathrm{H}), 7.11-7.04(\mathrm{~m}, 2 \mathrm{H}), 6.96(\mathrm{dd}, J=8.2,2.1 \mathrm{~Hz}, 1 \mathrm{H}), 6.51$ (s, 1H). ${ }^{13} \mathrm{C}$ NMR $\left(100 \mathrm{MHz}, \mathrm{CDCl}_{3}\right) \delta \mathrm{ppm} \mathrm{192.7,} \mathrm{182.9,} \mathrm{139.3,} \mathrm{139.1,} \mathrm{134.2,} \mathrm{133.7,} \mathrm{132.4,} \mathrm{132.2,} \mathrm{131.9,}$ 129.7, 129.6, 129.5, 129.5, 128.7, 126.9, 118.7, 118.6, 115.4, 106.0. HRMS MALDI (m/z): calcd for $\mathrm{C}_{22} \mathrm{H}_{13} \mathrm{Cl}_{2} \mathrm{NO}_{2}[\mathrm{M}+\mathrm{H}]^{+}:$394.0396, found: 394.0400 .

\section{1-(2-(Furan-2-yl)indolizin-3-yl)-2-phenylethane-1,2-dione (3r)}

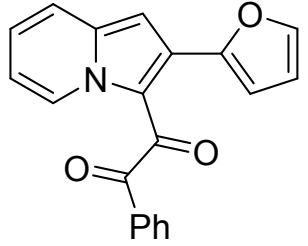

Flash column chromatography on silica gel (eluent: PE/EtOAc $=15 / 1, \mathrm{v} / \mathrm{v}$ ) to afford 3r. Yellow solid (83.5 mg, 53\%); mp 138-140 C; IR (KBr): 3052, 2920, 1682, 1574, 1236, 885, $645 \mathrm{~cm}^{-1} ;{ }^{1} \mathrm{H}$ NMR $\left(400 \mathrm{MHz}, \mathrm{CDCl}_{3}\right) \delta \mathrm{ppm} 10.60(\mathrm{~d}, J=7.0 \mathrm{~Hz}, 1 \mathrm{H})$, $8.62(\mathrm{~d}, J=7.2 \mathrm{~Hz}, 2 \mathrm{H}), 8.19(\mathrm{t}, J=7.4 \mathrm{~Hz}, 1 \mathrm{H}), 8.14(\mathrm{~s}, 1 \mathrm{H}), 8.08(\mathrm{t}, J=7.7 \mathrm{~Hz}, 2 \mathrm{H})$, $7.90-7.86(\mathrm{~m}, 1 \mathrm{H}), 7.58(\mathrm{t}, J=6.9 \mathrm{~Hz}, 1 \mathrm{H}), 7.42(\mathrm{~d}, J=1.3 \mathrm{~Hz}, 1 \mathrm{H}), 7.26(\mathrm{~s}, 1 \mathrm{H}), 7.09(\mathrm{~d}, J=3.2 \mathrm{~Hz}, 1 \mathrm{H})$, $6.90(\mathrm{dd}, J=3.4,1.8 \mathrm{~Hz}, 1 \mathrm{H}) .{ }^{13} \mathrm{C}$ NMR $\left(100 \mathrm{MHz}, \mathrm{CDCl}_{3}\right) \delta \mathrm{ppm} \mathrm{191.4,} \mathrm{183.2,} \mathrm{147.9,} \mathrm{142.7,} \mathrm{139.5,} \mathrm{134.0,}$ 133.6, 130.0, 129.4, 129.3, 128.5, 126.4, 118.6, 117.5, 115.0, 111.9, 110.1, 103.9. HRMS MALDI (m/z): calcd for $\mathrm{C}_{20} \mathrm{H}_{13} \mathrm{NO}_{3}[\mathrm{M}+\mathrm{H}]^{+}: 316.0968$, found: 316.0969 . 


\section{1-(2-(Benzo[d][1,3]dioxol-5-yl)indolizin-3-yl)-2-phenylethane-1,2-dione (3s)}

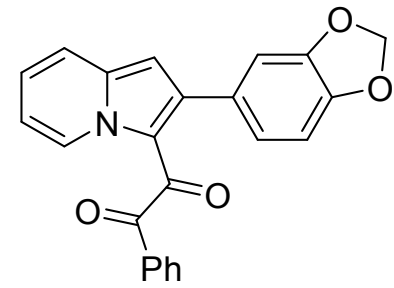

Flash column chromatography on silica gel (eluent: $\mathrm{PE} / \mathrm{EtOAc}=15 / 1, \mathrm{v} / \mathrm{v})$ to afford 3s. Brown solid (121.8 mg, 66\%); mp 119-121 ${ }^{\circ} \mathrm{C}$; IR (KBr): 3067, 2921, $1550,1469,1236,1040,716 \mathrm{~cm}^{-1} ;{ }^{1} \mathrm{H}$ NMR $\left(400 \mathrm{MHz}, \mathrm{CDCl}_{3}\right) \delta \mathrm{ppm} 10.06(\mathrm{~d}$, $J=7.1 \mathrm{~Hz}, 1 \mathrm{H}), 7.71(\mathrm{~d}, J=7.5 \mathrm{~Hz}, 2 \mathrm{H}), 7.57(\mathrm{~s}, 1 \mathrm{H}), 7.53(\mathrm{~s}, 1 \mathrm{H}), 7.37(\mathrm{~s}, 3 \mathrm{H})$, $7.04(\mathrm{~s}, 1 \mathrm{H}), 6.51(\mathrm{~d}, J=14.9 \mathrm{~Hz}, 3 \mathrm{H}), 6.41(\mathrm{~s}, 1 \mathrm{H}), 5.85(\mathrm{~s}, 2 \mathrm{H}) .{ }^{13} \mathrm{C} \mathrm{NMR}\left(100 \mathrm{MHz}, \mathrm{CDCl}_{3}\right) \delta \mathrm{ppm} \mathrm{192.4,}$ 183.2, 147.7, 147.1, 141.8, 139.4, 134.4, 133.7, 129.6, 128.4, 127.8, 126.6, 124.7, 118.5, 114.9, 110.7, 107.5, 105.8, 101.2. HRMS MALDI (m/z): calcd for $\mathrm{C}_{23} \mathrm{H}_{15} \mathrm{NO}_{4}[\mathrm{M}+\mathrm{Na}]^{+}: 392.0893$, found: 392.0890 .

\section{1-Phenyl-2-(2-propionylindolizin-3-yl)ethane-1,2-dione (3t)}

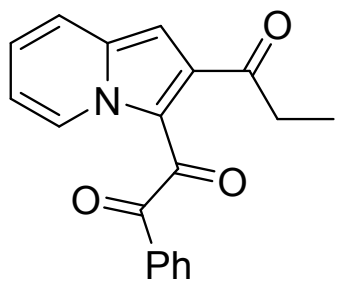

Flash column chromatography on silica gel (eluent: $\mathrm{PE} / \mathrm{EtOAc}=10 / 1, \mathrm{v} / \mathrm{v})$ to afford 3t. Brown oil (112.9 mg, 74\%), mp 134-136 ${ }^{\circ} \mathrm{C}$; IR (KBr): 3069, 2926, 1682, 1483, 1348, 1250, $726 \mathrm{~cm}^{-1} ;{ }^{1} \mathrm{H}$ NMR $\left(400 \mathrm{MHz}, \mathrm{CDCl}_{3}\right) \delta \mathrm{ppm} \delta 9.38(\mathrm{~d}, J=6.9 \mathrm{~Hz}$, $1 \mathrm{H}), 7.64(\mathrm{~d}, J=9.0 \mathrm{~Hz}, 1 \mathrm{H}), 7.43-7.37(\mathrm{~m}, 1 \mathrm{H}), 7.31-7.16(\mathrm{~m}, 5 \mathrm{H}), 7.03(\mathrm{~d}, J=$ $5.0 \mathrm{~Hz}, 1 \mathrm{H}), 6.91(\mathrm{~s}, 1 \mathrm{H}), 3.67(\mathrm{q}, 2 \mathrm{H}), 1.42(\mathrm{~d}, J=7.2 \mathrm{~Hz}, 3 \mathrm{H}) .{ }^{13} \mathrm{C}$ NMR $\left(100 \mathrm{MHz}, \mathrm{CDCl}_{3}\right) \delta \mathrm{ppm} 197.2$, $194.3,186.3,186.2,143.3,139.7,131.4,128.6,128.5,128.5,128.2,128.0,127.7,126.2,126.0,125.8,125.8$, 120.7, 119.8, 116.5, 116.4, 100.9, 100.5, 81.1, 56.3, 56.2, 15.8, 9.0. HRMS MALDI (m/z): calcd for $\mathrm{C}_{19} \mathrm{H}_{15} \mathrm{NO}_{3}[\mathrm{M}+\mathrm{Na}]^{+}:$328.0944, found: 328.0948 .

\section{3-(2-Oxo-2-phenylacetyl)indolizine-1-carbonitrile (3u)}<smiles>N#Cc1cc(C(=O)C(=O)c2ccccc2)n2ccccc12</smiles>

Flash column chromatography on silica gel (eluent: PE/EtOAc $=10 / 1, \mathrm{v} / \mathrm{v}$ ) to afford $\mathbf{3 u}$. Yellow solid (110.0 mg, 89\%), mp 129-131 ${ }^{\circ} \mathrm{C}$; IR (KBr): 3075, 1777, 1243, 1165, 1051, 760, $687 \mathrm{~cm}^{-1} ;{ }^{1} \mathrm{H}$ NMR $\left(400 \mathrm{MHz}, \mathrm{CDCl}_{3}\right) \delta$ ppm $9.95(\mathrm{~d}, J=7.0 \mathrm{~Hz}, 1 \mathrm{H}), 8.03(\mathrm{~d}, J=$ $6.9 \mathrm{~Hz}, 2 \mathrm{H}), 7.84(\mathrm{~d}, J=8.8 \mathrm{~Hz}, 1 \mathrm{H}), 7.75(\mathrm{~s}, 1 \mathrm{H}), 7.65(\mathrm{t}, J=7.4 \mathrm{~Hz}, 1 \mathrm{H}), 7.60-7.56(\mathrm{~m}$, 1H), $7.50(\mathrm{t}, J=7.7 \mathrm{~Hz}, 2 \mathrm{H}), 7.26-7.21(\mathrm{~m}, 1 \mathrm{H}) .{ }^{13} \mathrm{C} \mathrm{NMR}\left(100 \mathrm{MHz}, \mathrm{CDCl}_{3}\right) \delta \mathrm{ppm}$ 191.8, 181.6, 141.8, 134.9, 132.9, 130.3, 130.3, 129.9, 129.3, 129.0, 120.6, 117.8, 117.0, 114.4, 86.9. HRMS MALDI (m/z): calcd for $\mathrm{C}_{17} \mathrm{H}_{10} \mathrm{~N}_{2} \mathrm{O}_{2}[\mathrm{M}+\mathrm{Na}]^{+}:$297.0635, found: 297.0644 .

\section{Methyl 3-(2-oxo-2-phenylacetyl)indolizine-1-carboxylate (3v)}




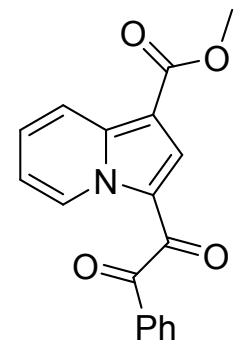

Flash column chromatography on silica gel (eluent: PE/EtOAc $=10 / 1, \mathrm{v} / \mathrm{v}$ ) to afford $\mathbf{3 v}$. Yellow solid (115.2 mg, 75\%); mp 156-158 ${ }^{\circ} \mathrm{C}$; IR (KBr): 3055, 1702, 1520, 1365, 1047 , 732, $691 \mathrm{~cm}^{-1} ;{ }^{1} \mathrm{H}$ NMR (400 MHz, $\left.\mathrm{CDCl}_{3}\right) \delta \mathrm{ppm} 9.93(\mathrm{~d}, J=7.0 \mathrm{~Hz}, 1 \mathrm{H}), 8.40$ (ddq, $J=$ 8.8, 3.0, 1.5 Hz, 1H), $8.04(\mathrm{~d}, J=7.4 \mathrm{~Hz}, 2 \mathrm{H}), 7.90(\mathrm{~s}, 1 \mathrm{H}), 7.62(\mathrm{t}, J=7.3 \mathrm{~Hz}, 1 \mathrm{H}), 7.48(\mathrm{t}$, $J=7.7 \mathrm{~Hz}, 3 \mathrm{H}), 7.17(\mathrm{~d}, J=6.1 \mathrm{~Hz}, 1 \mathrm{H}), 3.86(\mathrm{~s}, 3 \mathrm{H}) .{ }^{13} \mathrm{C} \mathrm{NMR}\left(100 \mathrm{MHz}, \mathrm{CDCl}_{3}\right) \delta \mathrm{ppm}$ $192.5,182.2,163.9,140.8,134.7,133.2,130.3,129.7,129.6,129.2,128.9,120.2,119.8,116.4,107.8,51.5$ HRMS MALDI (m/z): calcd for $\mathrm{C}_{18} \mathrm{H}_{13} \mathrm{NO}_{4}[\mathrm{M}+\mathrm{Na}]^{+}: 330.0737$, found: 330.0733 .

\section{1-(2-Phenylindolizin-3-yl)-2-(p-tolyl)ethane-1,2-dione (4a)}

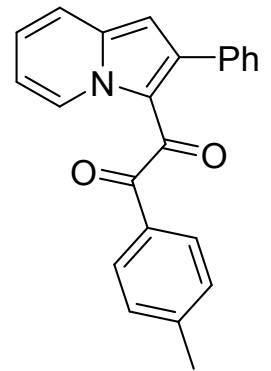

Flash column chromatography on silica gel (eluent: PE/EtOAc $=15 / 1, \mathrm{v} / \mathrm{v}$ ) to afford $\mathbf{4 a}$. Green solid (144.1 mg, 85\%), mp 157-159 ${ }^{\circ} \mathrm{C}$; IR (KBr): 3052, 2921, 1675, 1419, 1246, 733, $689 \mathrm{~cm}^{-1} ;{ }^{1} \mathrm{H}$ NMR (400 MHz, $\left.\mathrm{CDCl}_{3}\right) \delta \mathrm{ppm} 10.08(\mathrm{~d}, J=5.9 \mathrm{~Hz}, 1 \mathrm{H}), 7.60-7.53$ $(\mathrm{m}, 3 \mathrm{H}), 7.33(\mathrm{~d}, J=6.9 \mathrm{~Hz}, 1 \mathrm{H}), 7.14(\mathrm{~d}, J=8.3 \mathrm{~Hz}, 3 \mathrm{H}), 7.09(\mathrm{~d}, J=9.7 \mathrm{~Hz}, 2 \mathrm{H}), 7.02$ $(\mathrm{d}, J=6.8 \mathrm{~Hz}, 3 \mathrm{H}), 6.52(\mathrm{~s}, 1 \mathrm{H}), 2.38(\mathrm{~s}, 3 \mathrm{H}) .{ }^{13} \mathrm{C} \mathrm{NMR}\left(100 \mathrm{MHz}, \mathrm{CDCl}_{3}\right) \delta \mathrm{ppm} \mathrm{192.2,}$ $183.5,144.6,142.1,139.3,134.3,131.7,130.4,129.8,129.6,129.1,127.9,127.6,126.5,118.8,118.5,114.8$, 106.0, 21.9. HRMS MALDI (m/z): calcd for $\mathrm{C}_{23} \mathrm{H}_{17} \mathrm{NO}_{2}[\mathrm{M}+\mathrm{Na}]^{+}: 362.1152$, found: 362.1152

\section{1-(2-Phenylindolizin-3-yl)-2-(m-tolyl)ethane-1,2-dione (4b)}

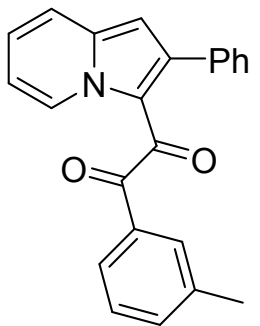

Flash column chromatography on silica gel (eluent: PE/EtOAc $=15 / 1, \mathrm{v} / \mathrm{v}$ ) to afford $\mathbf{4 b}$. Green solid (140.7 mg, 83\%), mp 126-128 ${ }^{\circ} \mathrm{C}$; IR (KBr): 3057, 2924, 1679, 1415, 1240, 731, $692 \mathrm{~cm}^{-1} ;{ }^{1} \mathrm{H}$ NMR (400 MHz, $\left.\mathrm{CDCl}_{3}\right) \delta \mathrm{ppm} 10.84(\mathrm{~d}, J=7.0 \mathrm{~Hz}, 1 \mathrm{H}), 8.31(\mathrm{~d}, J=$ $8.4 \mathrm{~Hz}, 1 \mathrm{H}), 8.23-8.17(\mathrm{~m}, 2 \mathrm{H}), 8.05(\mathrm{~d}, J=5.4 \mathrm{~Hz}, 2 \mathrm{H}), 7.93(\mathrm{dt}, J=13.7,7.5 \mathrm{~Hz}, 3 \mathrm{H})$, $7.84(\mathrm{~d}, J=6.8 \mathrm{~Hz}, 2 \mathrm{H}), 7.76(\mathrm{t}, J=7.4 \mathrm{~Hz}, 3 \mathrm{H}), 3.05(\mathrm{~s}, 3 \mathrm{H}) .{ }^{13} \mathrm{C} \mathrm{NMR}(100 \mathrm{MHz}$, $\left.\mathrm{CDCl}_{3}\right) \delta \mathrm{ppm} 192.7,183.2,142.0,139.2,138.0,134.4,134.1,134.0,130.3,129.7,129.3,128.1,127.7$, 127.5, 126.8, 126.5, 118.6, 118.4, 114.8, 106.0, 21.2. HRMS MALDI (m/z): calcd for $\mathrm{C}_{23} \mathrm{H}_{17} \mathrm{NO}_{2}[\mathrm{M}+\mathrm{H}]^{+}$: 340.1332, found: 340.1336 .

\section{1-(3-Bromophenyl)-2-(2-phenylindolizin-3-yl)ethane-1,2-dione (4c)}




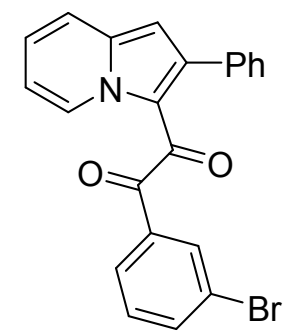

Flash column chromatography on silica gel (eluent: PE/EtOAc $=15 / 1, \mathrm{v} / \mathrm{v}$ ) to afford 4c. Yellow solid (177.3 mg, 88\%), mp 137-139 ${ }^{\circ} \mathrm{C}$; IR (KBr): 3067, 2912, 1588, 1412, 1240, 733, $699 \mathrm{~cm}^{-1} ;{ }^{1} \mathrm{H}$ NMR $\left(400 \mathrm{MHz}, \mathrm{CDCl}_{3}\right) \delta \mathrm{ppm} 10.03(\mathrm{~d}, J=6.9 \mathrm{~Hz}, 1 \mathrm{H}), 7.68(\mathrm{t}, J=$ $1.8 \mathrm{~Hz}, 1 \mathrm{H}), 7.61-7.54(\mathrm{~m}, 3 \mathrm{H}), 7.33(\mathrm{~d}, J=6.9 \mathrm{~Hz}, 1 \mathrm{H}), 7.17(\mathrm{t}, J=7.9 \mathrm{~Hz}, 2 \mathrm{H}), 7.04$

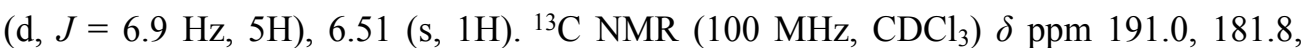
$142.0,139.4,136.3,135.7,133.9,131.9,130.3,129.8,129.3,128.0,127.9,127.6,126.8,122.5,118.5,118.4$, 115.0, 106.2. HRMS MALDI (m/z): calcd for $\mathrm{C}_{22} \mathrm{H}_{14} \mathrm{BrNO}_{2}[\mathrm{M}+\mathrm{H}]^{+}:$404.0281, found: 404.0283.

\section{1-(2-Fluorophenyl)-2-(2-phenylindolizin-3-yl)ethane-1,2-dione (4d)}<smiles>O=C(C(=O)c1c(-c2ccccc2)cc2ccccn12)c1ccccc1</smiles>

Flash column chromatography on silica gel (eluent: PE/EtOAc $=15 / 1, \mathrm{v} / \mathrm{v}$ ) to afford $\mathbf{4 d}$. Green solid (149.2 mg, 87\%); mp 149-151 ${ }^{\circ} \mathrm{C}$; IR (KBr): 3053, 2931, 1594, 1425, 1246, 858, $747 \mathrm{~cm}^{-1} ;{ }^{1} \mathrm{H}$ NMR $\left(400 \mathrm{MHz}, \mathrm{CDCl}_{3}\right) \delta \mathrm{ppm} 9.95(\mathrm{~d}, J=6.9 \mathrm{~Hz}, 1 \mathrm{H}), 7.56(\mathrm{~d}, J=$ $7.5 \mathrm{~Hz}, 1 \mathrm{H}), 7.52(\mathrm{~d}, J=8.8 \mathrm{~Hz}, 1 \mathrm{H}), 7.45(\mathrm{~d}, J=8.1 \mathrm{~Hz}, 1 \mathrm{H}), 7.22(\mathrm{t}, J=7.5 \mathrm{~Hz}, 3 \mathrm{H})$, $7.09(\mathrm{~d}, J=7.4 \mathrm{~Hz}, 1 \mathrm{H}), 7.03(\mathrm{q}, J=8.0,7.4 \mathrm{~Hz}, 4 \mathrm{H}), 6.94(\mathrm{t}, J=7.0 \mathrm{~Hz}, 1 \mathrm{H}), 6.51(\mathrm{~s}$, 1H). ${ }^{13} \mathrm{C}$ NMR $\left(100 \mathrm{MHz}, \mathrm{CDCl}_{3}\right) \delta \mathrm{ppm} 188.7,182.3,162.0(J=257.6 \mathrm{~Hz}), 141.8,139.4,135.7(J=9.1 \mathrm{~Hz})$, $134.6,131.3,131.1,130.1,129.5,128.9,127.8,126.4,124.4(J=3.4 \mathrm{~Hz}), 118.5,116.4,116.2,114.8,105.8$. HRMS MALDI (m/z): calcd for $\mathrm{C}_{22} \mathrm{H}_{14} \mathrm{FNO}_{2}[\mathrm{M}+\mathrm{H}]^{+}: 344.1081$, found: 344.1084 .

\section{1-(3,4-Dichlorophenyl)-2-(2-phenylindolizin-3-yl)ethane-1,2-dione (4e)}

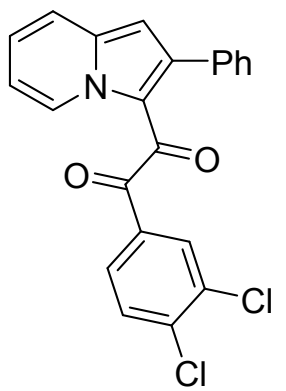

Flash column chromatography on silica gel (eluent: PE/EtOAc $=15 / 1, \mathrm{v} / \mathrm{v}$ ) to afford $\mathbf{4 e}$. Green solid (159.2 mg, 81\%); mp 125-127 ${ }^{\circ} \mathrm{C}$; IR (KBr): 3055, 2934, 1685, 1419, 1236, 735, $683 \mathrm{~cm}^{-1} ;{ }^{1} \mathrm{H}$ NMR (400 MHz, $\left.\mathrm{CDCl}_{3}\right) \delta \mathrm{ppm} 10.02(\mathrm{~d}, J=7.0 \mathrm{~Hz}, 1 \mathrm{H}), 7.65(\mathrm{~s}$, $1 \mathrm{H}), 7.58(\mathrm{~d}, J=8.7 \mathrm{~Hz}, 1 \mathrm{H}), 7.46(\mathrm{~d}, J=8.3 \mathrm{~Hz}, 1 \mathrm{H}), 7.36(\mathrm{dd}, J=21.6,8.0 \mathrm{~Hz}, 2 \mathrm{H})$, $7.21(\mathrm{q}, J=4.0 \mathrm{~Hz}, 1 \mathrm{H}), 7.06(\mathrm{~d}, J=3.7 \mathrm{~Hz}, 5 \mathrm{H}), 6.53(\mathrm{~s}, 1 \mathrm{H}) .{ }^{13} \mathrm{C}$ NMR $(100 \mathrm{MHz}$, $\left.\mathrm{CDCl}_{3}\right) \delta \mathrm{ppm} 190.1,181.4,142.1,139.6,138.0,134.0,133.7,133.0,130.9,130.4$, $130.4,129.5,128.4,128.1,127.7,127.1,118.6,118.5,115.1,106.3$. HRMS MALDI (m/z): calcd for $\mathrm{C}_{22} \mathrm{H}_{13} \mathrm{Cl}_{2} \mathrm{NO}_{2}[\mathrm{M}+\mathrm{H}]^{+}:$394.0396, found: 394.0394 .

\section{1-(3,4-Dimethoxyphenyl)-2-(2-phenylindolizin-3-yl)ethane-1,2-dione (4f)}




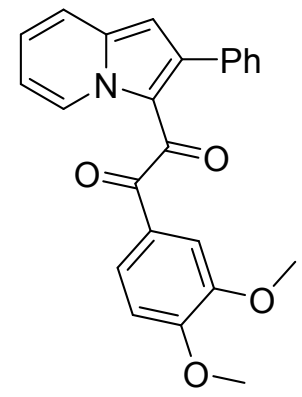

Flash column chromatography on silica gel (eluent: PE/EtOAc $=15 / 1, \mathrm{v} / \mathrm{v}$ ) to afford $\mathbf{4 f}$. Green solid (150.2 mg, 78\%); mp 205-207 ${ }^{\circ} \mathrm{C}$; IR (KBr): 3068, 2920, 1544, 1020, 735 , $685 \mathrm{~cm}^{-1} ;{ }^{1} \mathrm{H}$ NMR (400 MHz, $\left.\mathrm{CDCl}_{3}\right) \delta \mathrm{ppm} 10.06(\mathrm{~d}, J=7.0 \mathrm{~Hz}, 1 \mathrm{H}), 7.56(\mathrm{~d}, J=$ $8.7 \mathrm{~Hz}, 1 \mathrm{H}), 7.36(\mathrm{~d}, J=10.3 \mathrm{~Hz}, 1 \mathrm{H}), 7.30(\mathrm{t}, J=7.7 \mathrm{~Hz}, 1 \mathrm{H}), 7.13(\mathrm{~d}, J=7.1 \mathrm{~Hz}$, 1H), $7.07(\mathrm{~d}, J=6.8 \mathrm{~Hz}, 2 \mathrm{H}), 7.05-7.00(\mathrm{~m}, 4 \mathrm{H}), 6.76(\mathrm{~d}, J=8.4 \mathrm{~Hz}, 1 \mathrm{H}), 6.50(\mathrm{~s}$, 1H), $3.91(\mathrm{~s}, 3 \mathrm{H}), 3.79(\mathrm{~s}, 3 \mathrm{H}) .{ }^{13} \mathrm{C} \mathrm{NMR}\left(100 \mathrm{MHz}, \mathrm{CDCl}_{3}\right) \delta \mathrm{ppm} 191.5,183.3,153.8$, $148.9,142.0,139.2,134.4,130.3,129.4,127.7,127.6,127.5,126.4,125.5,118.8,118.4,114.8,110.3,109.8$, 106.0, 56.2, 56.0. HRMS MALDI (m/z): calcd for $\mathrm{C}_{24} \mathrm{H}_{19} \mathrm{NO}_{4}[\mathrm{M}+\mathrm{H}]^{+}:$386.1387, found: 386.1386 .

\section{1-(Furan-2-yl)-2-(2-phenylindolizin-3-yl)ethane-1,2-dione (4g)}<smiles>O=C(C(=O)c1c(-c2ccccc2)cc2ccccn12)c1ccco1</smiles>

Flash column chromatography on silica gel (eluent: PE/EtOAc $=15 / 1, \mathrm{v} / \mathrm{v}$ ) to afford $\mathbf{4 g}$. Green solid (99.3 mg, 63\%), mp 173-175 C; IR (KBr): 3051, 2923, 1665, 1419, 1236, 1020, $731 \mathrm{~cm}^{-1} ;{ }^{1} \mathrm{H}$ NMR (400 MHz, $\left.\mathrm{CDCl}_{3}\right) \delta \mathrm{ppm} 9.95(\mathrm{~s}, 1 \mathrm{H}), 7.59-7.45(\mathrm{~m}, 2 \mathrm{H})$, $7.24-6.95(\mathrm{~m}, 8 \mathrm{H}), 6.50(\mathrm{dd}, J=30.4,10.2 \mathrm{~Hz}, 2 \mathrm{H}) .{ }^{13} \mathrm{C} \mathrm{NMR}\left(100 \mathrm{MHz}, \mathrm{CDCl}_{3}\right) \delta$ ppm 181.1, 180.3, 151.0, 147.4, 142.2, 139.5, 134.4, 129.9, 129.4, 128.1, 127.7, 126.7, 120.4, 118.5, 118.5, 114.9, 112.6, 106.0. HRMS MALDI (m/z): calcd for $\mathrm{C}_{20} \mathrm{H}_{13} \mathrm{NO}_{3}[\mathrm{M}+\mathrm{Na}]^{+}:$338.0788, found: 338.0792 .

\section{1-(2-Phenylindolizin-3-yl)propane-1,2-dione (4h)}<smiles>CC(=O)C(=O)c1c(-c2ccccc2)cc2ccccn12</smiles>

Flash column chromatography on silica gel (eluent: PE/EtOAc $=15 / 1, \mathrm{v} / \mathrm{v}$ ) to afford 4h. Green solid (72.3 mg, 55\%), mp 110-112 ${ }^{\circ} \mathrm{C}$; IR (KBr): 3069, 2921, 1702, 1581, 1240, 861, $760 \mathrm{~cm}^{-1} ;{ }^{1} \mathrm{H} \mathrm{NMR}\left(400 \mathrm{MHz}, \mathrm{CDCl}_{3}\right) \delta \mathrm{ppm} 9.84(\mathrm{~d}, J=6.9 \mathrm{~Hz}, 1 \mathrm{H})$, $7.56(\mathrm{~d}, J=8.4 \mathrm{~Hz}, 1 \mathrm{H}), 7.39(\mathrm{dd}, J=4.3,2.2 \mathrm{~Hz}, 3 \mathrm{H}), 7.36-7.34(\mathrm{~m}, 2 \mathrm{H}), 7.29$ (d, $J=7.4 \mathrm{~Hz}, 1 \mathrm{H}), 6.97(\mathrm{t}, J=6.8 \mathrm{~Hz}, 1 \mathrm{H}), 6.57(\mathrm{~d}, J=1.5 \mathrm{~Hz}, 1 \mathrm{H}), 2.15(\mathrm{~s}, 3 \mathrm{H}) .{ }^{13} \mathrm{C} \mathrm{NMR}\left(100 \mathrm{MHz} \mathrm{CDCl}_{3}\right)$ $\delta$ ppm 200.4, 183.4, 141.6, 139.3, 135.3, 129.9, 129.1, 128.4, 128.3, 126.3, 118.5, 117.2, 114.70, 105.7, 26.3. HRMS MALDI (m/z): calcd for $\mathrm{C}_{17} \mathrm{H}_{13} \mathrm{NO}_{2}[\mathrm{M}+\mathrm{Na}]^{+}: 286.0839$, found:286.0835. 


\section{NMR spectra for new compounds}

${ }^{1} \mathrm{H}$ NMR (400 MHz, $\mathrm{CDCl}_{3}$ ) spectrum of compound 3a

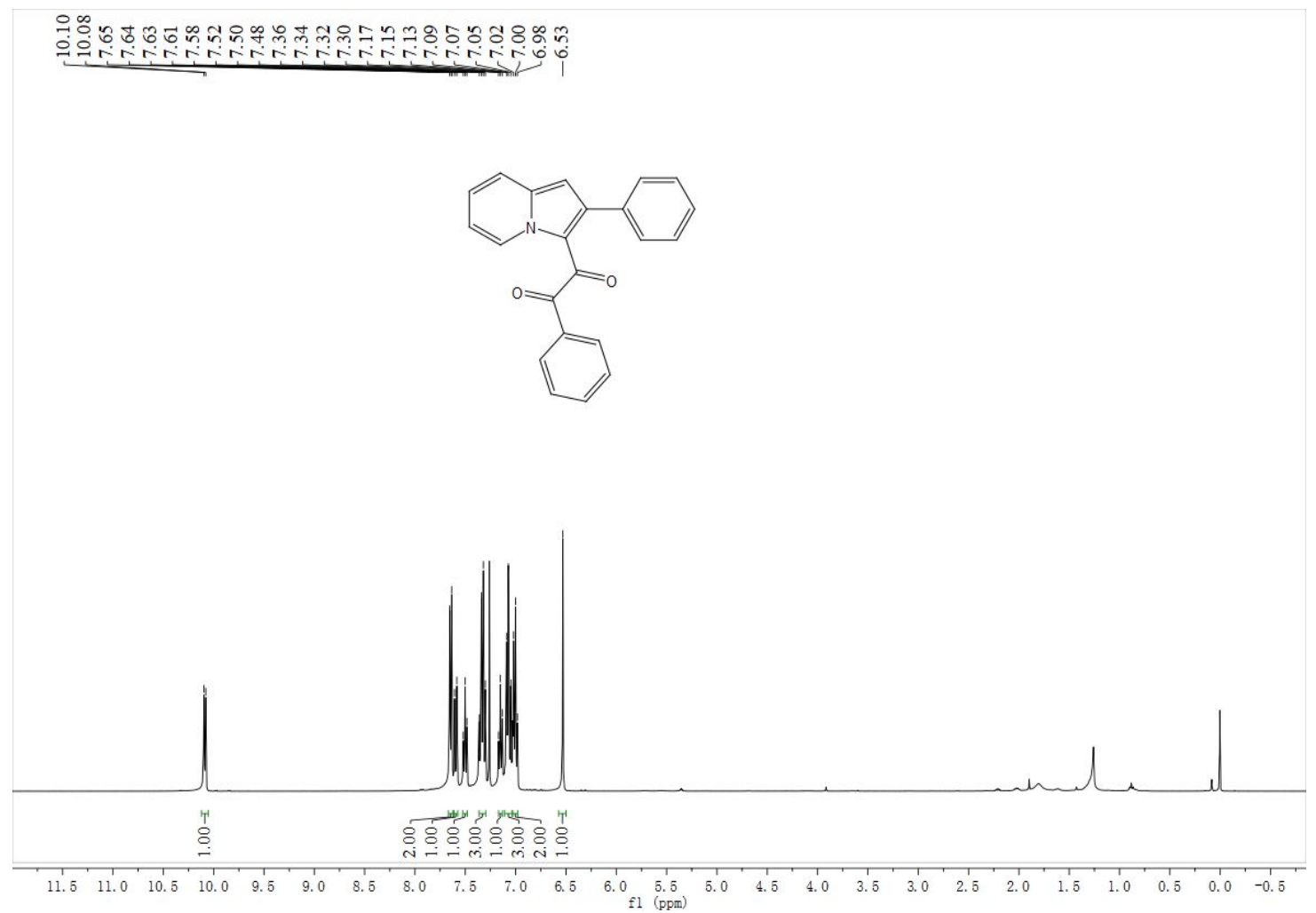

${ }^{13} \mathrm{C}$ NMR $\left(100 \mathrm{MHz}, \mathrm{CDCl}_{3}\right)$ spectrum of compound 3a
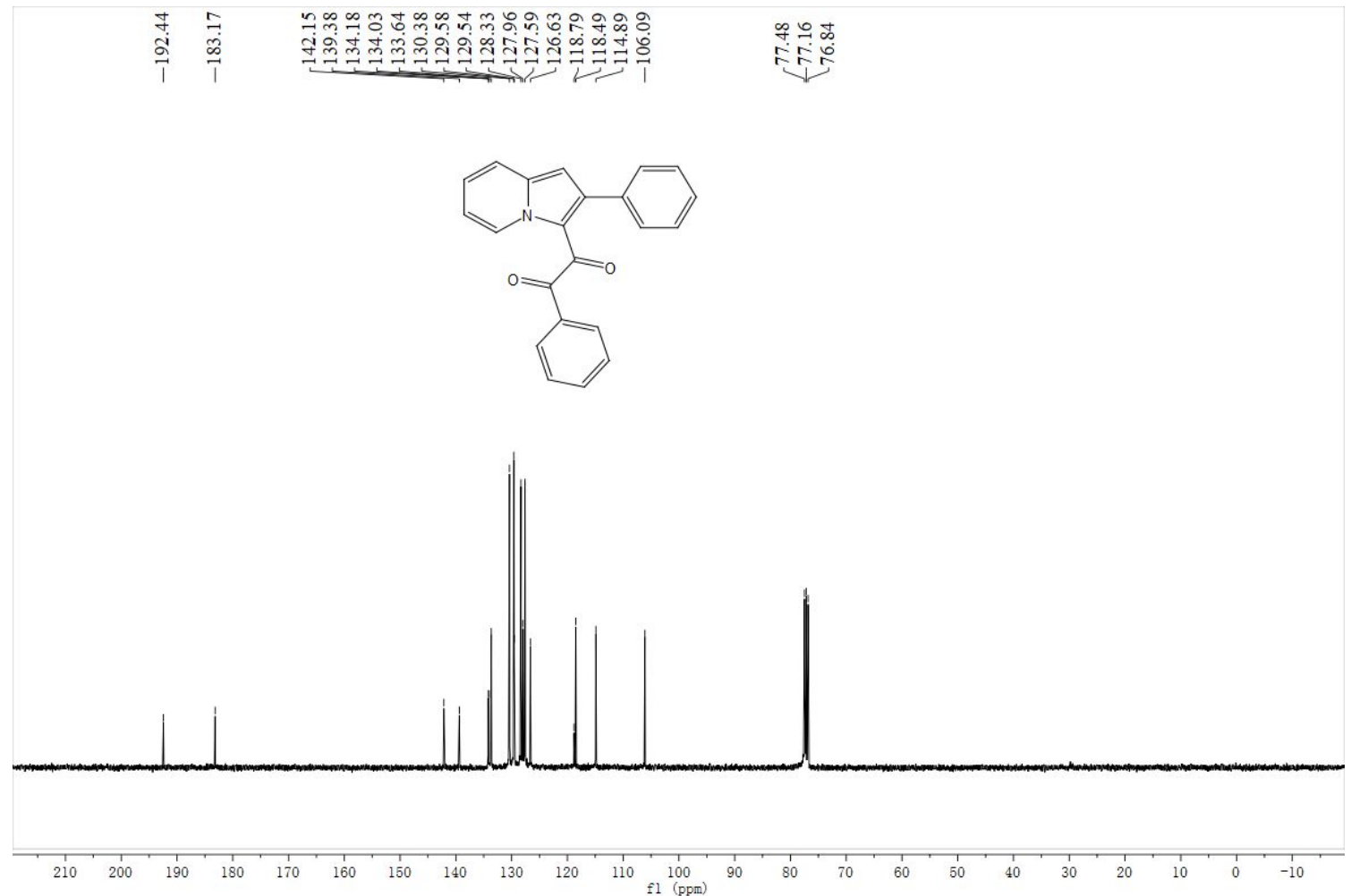
${ }^{1} \mathrm{H}$ NMR (400 MHz, $\left.\mathrm{CDCl}_{3}\right)$ spectrum of compound $\mathbf{3 b}$

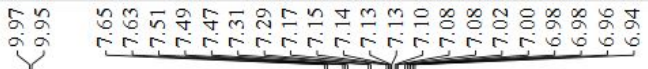
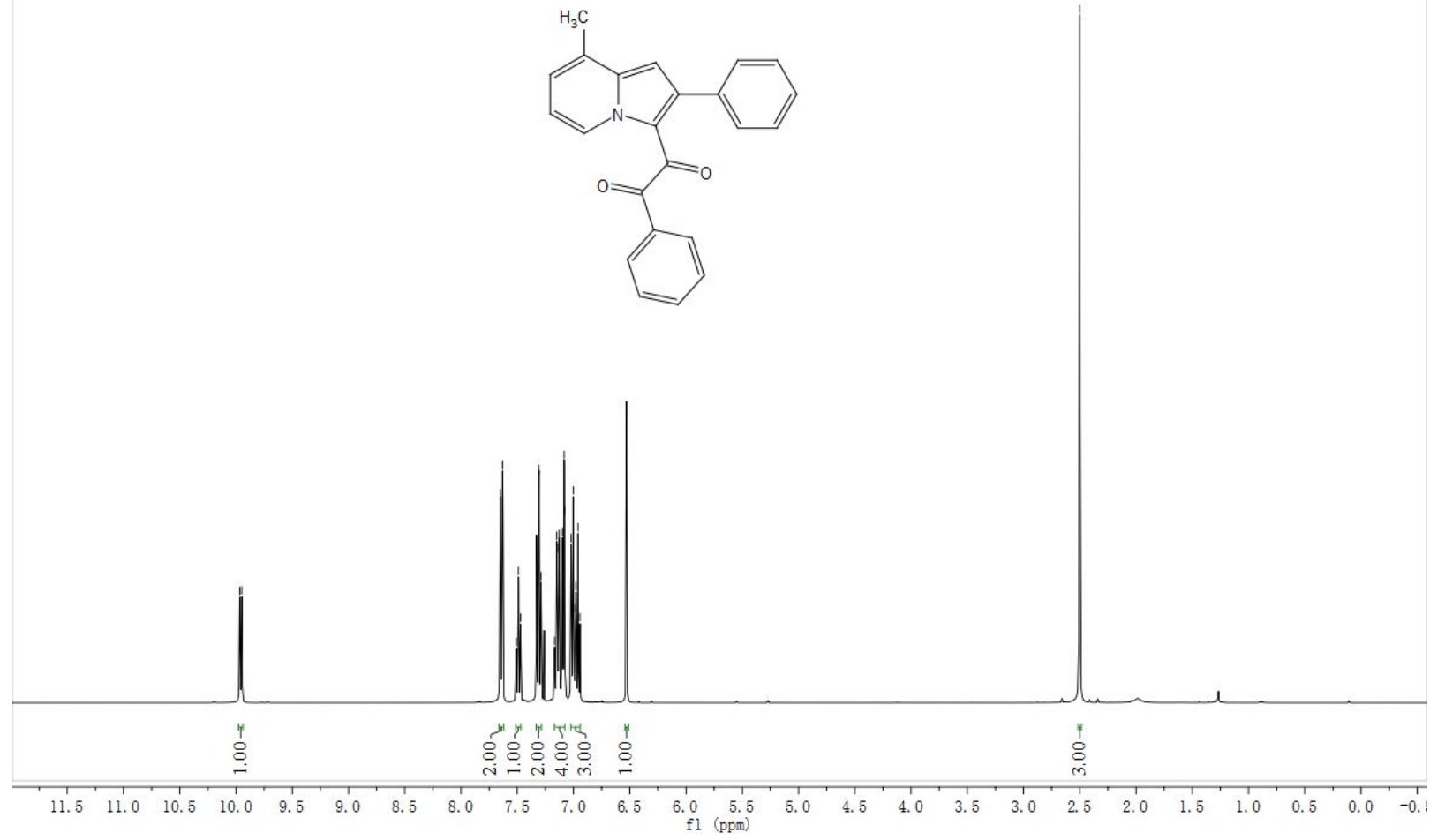

${ }^{13} \mathrm{C}$ NMR $\left(100 \mathrm{MHz}, \mathrm{CDCl}_{3}\right)$ spectrum of compound $\mathbf{3 b}$

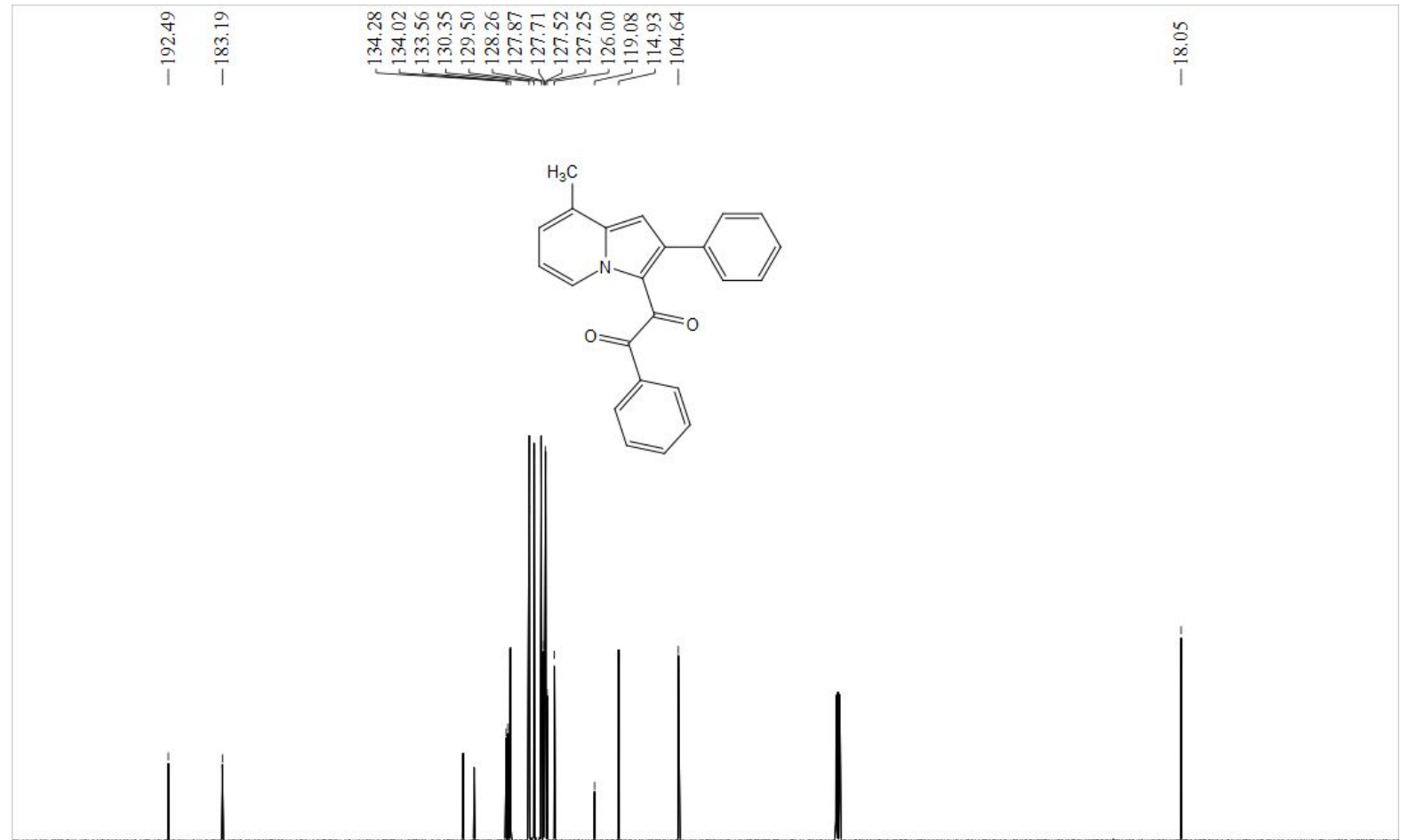

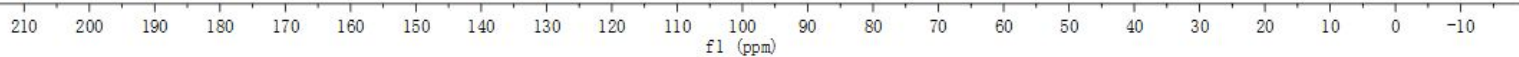


${ }^{1} \mathrm{H}$ NMR (400 MHz, $\mathrm{CDCl}_{3}$ ) spectrum of compound 3c

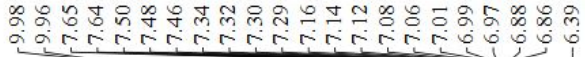
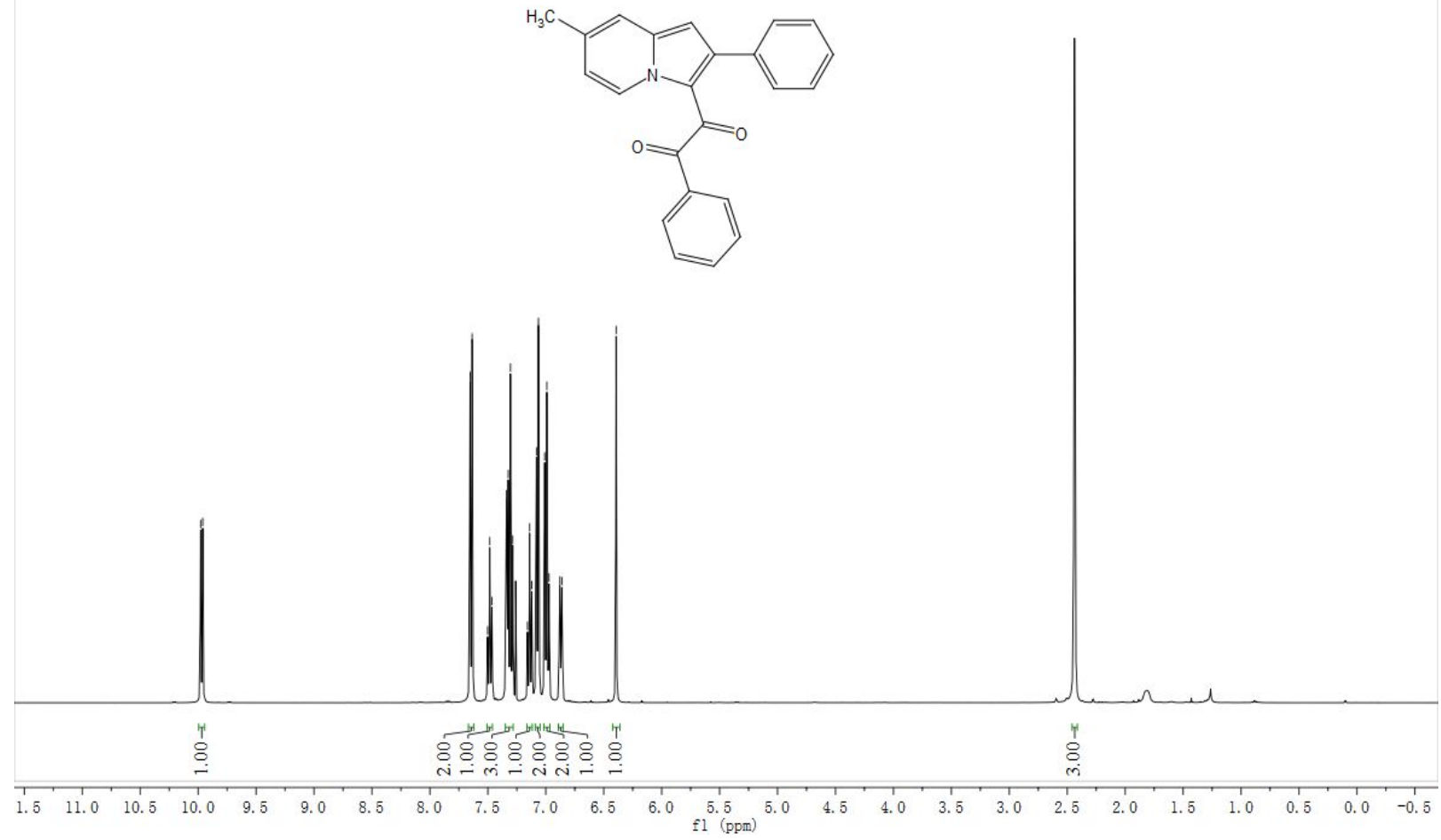

${ }^{13} \mathrm{C}$ NMR $\left(100 \mathrm{MHz}, \mathrm{CDCl}_{3}\right)$ spectrum of compound 3c

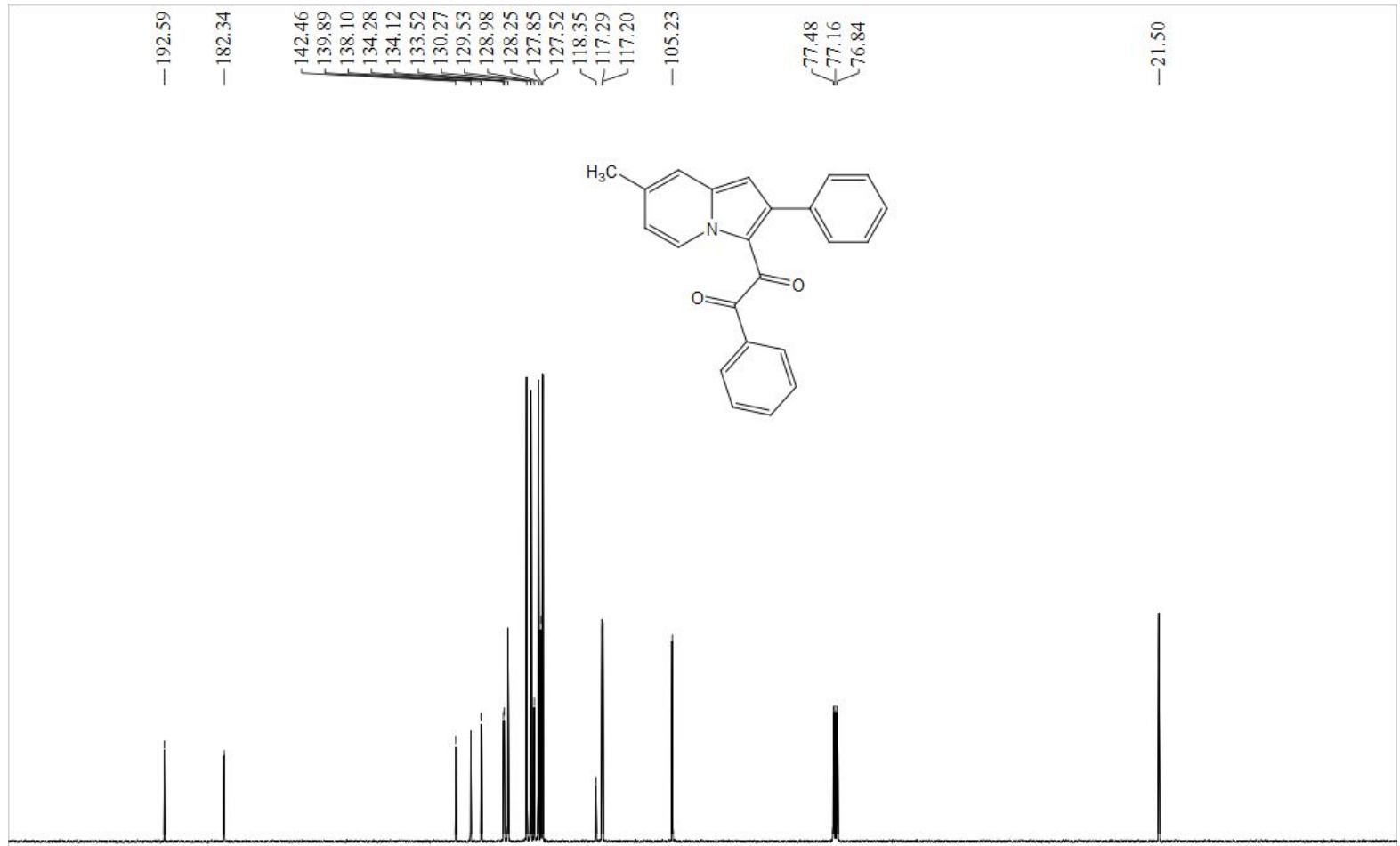

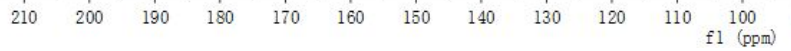


${ }^{1} \mathrm{H}$ NMR (400 MHz, $\mathrm{CDCl}_{3}$ ) spectrum of compound 3d

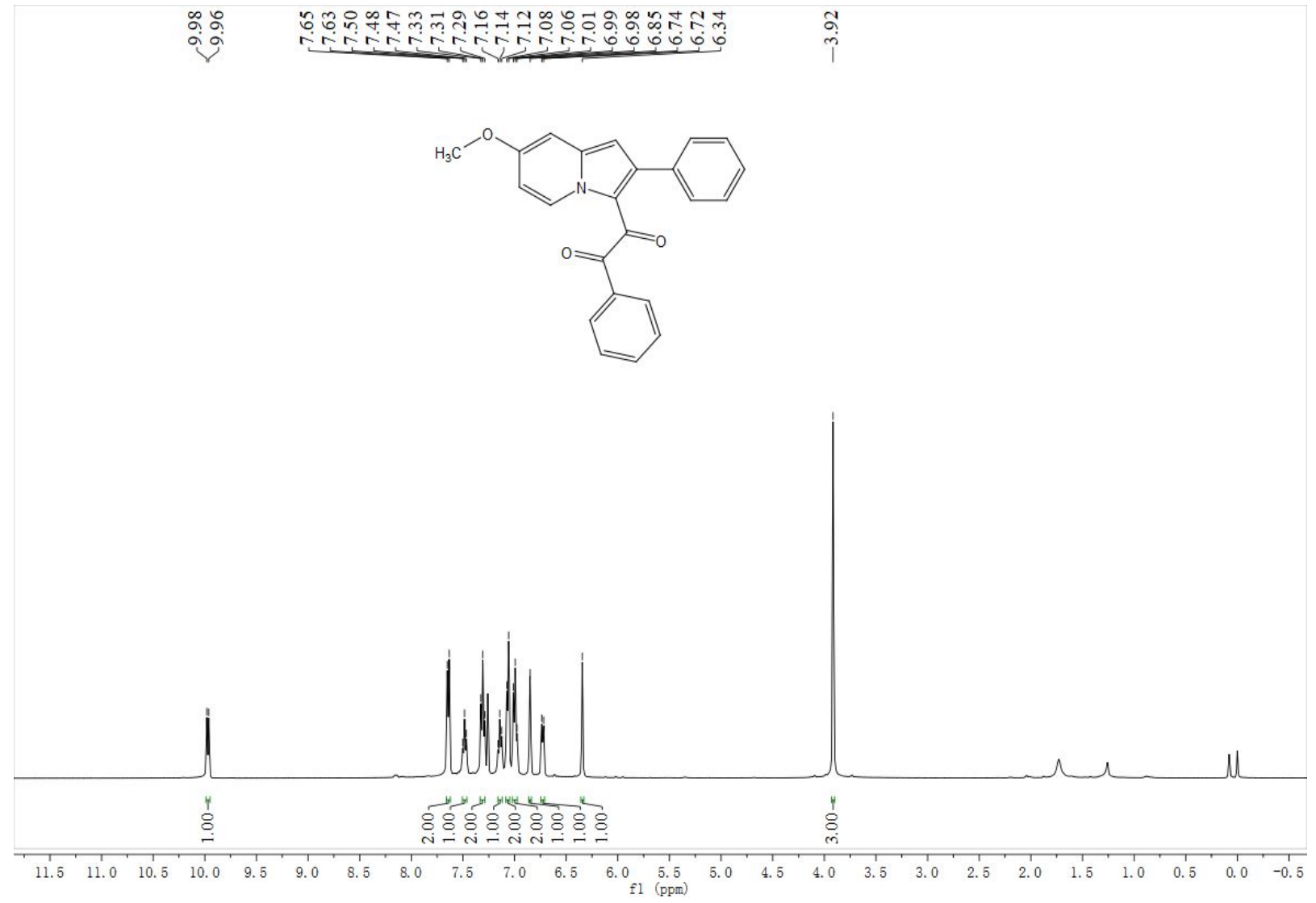

${ }^{13} \mathrm{C}$ NMR $\left(100 \mathrm{MHz}, \mathrm{CDCl}_{3}\right)$ spectrum of compound 3d

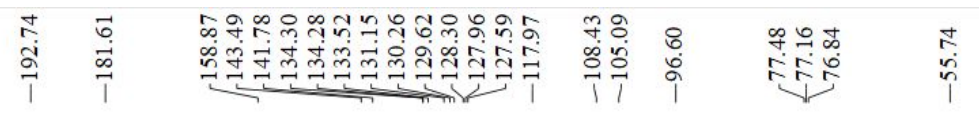

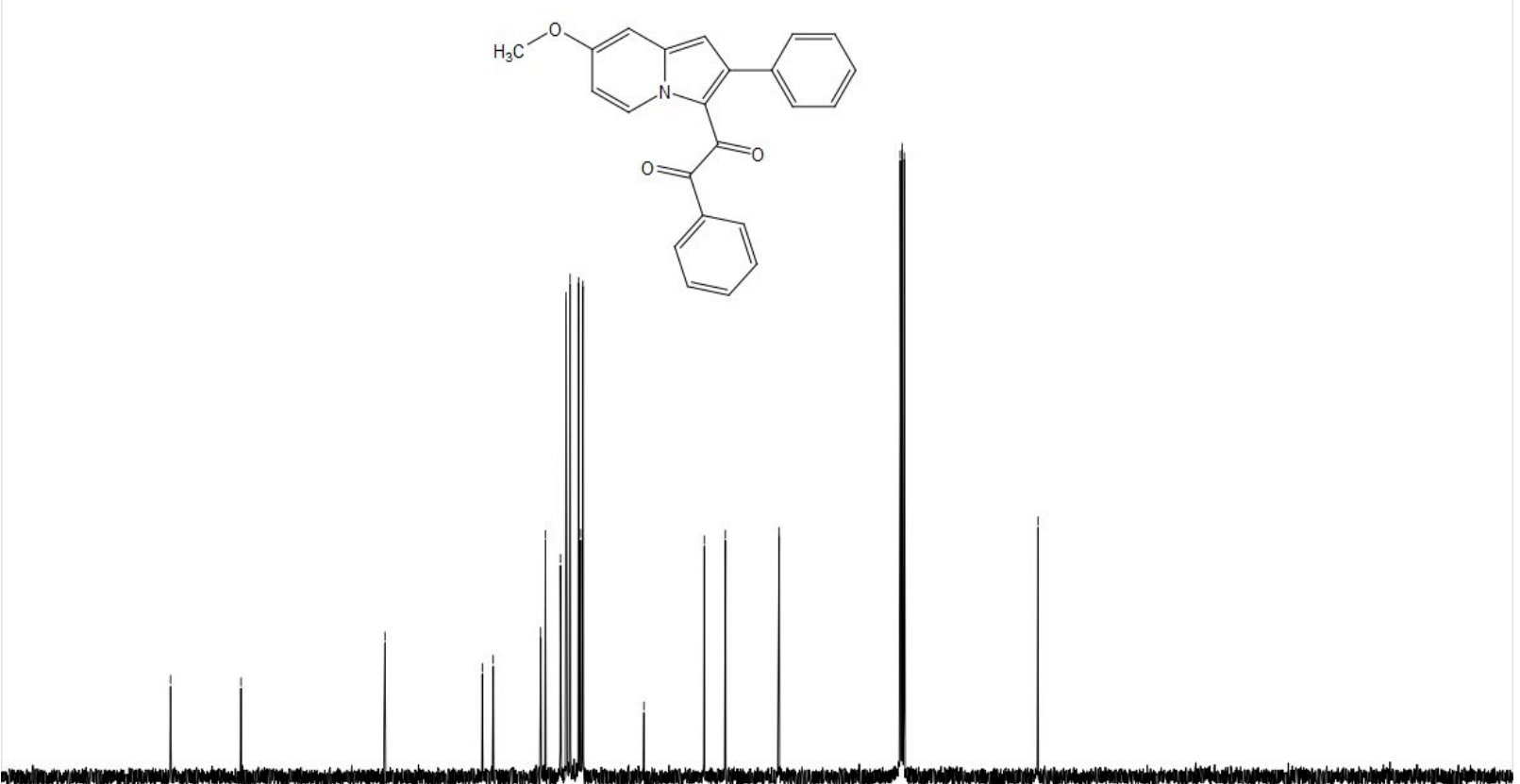

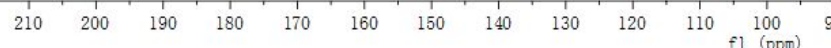


${ }^{1} \mathrm{H}$ NMR (400 MHz, $\mathrm{CDCl}_{3}$ ) spectrum of compound 3e

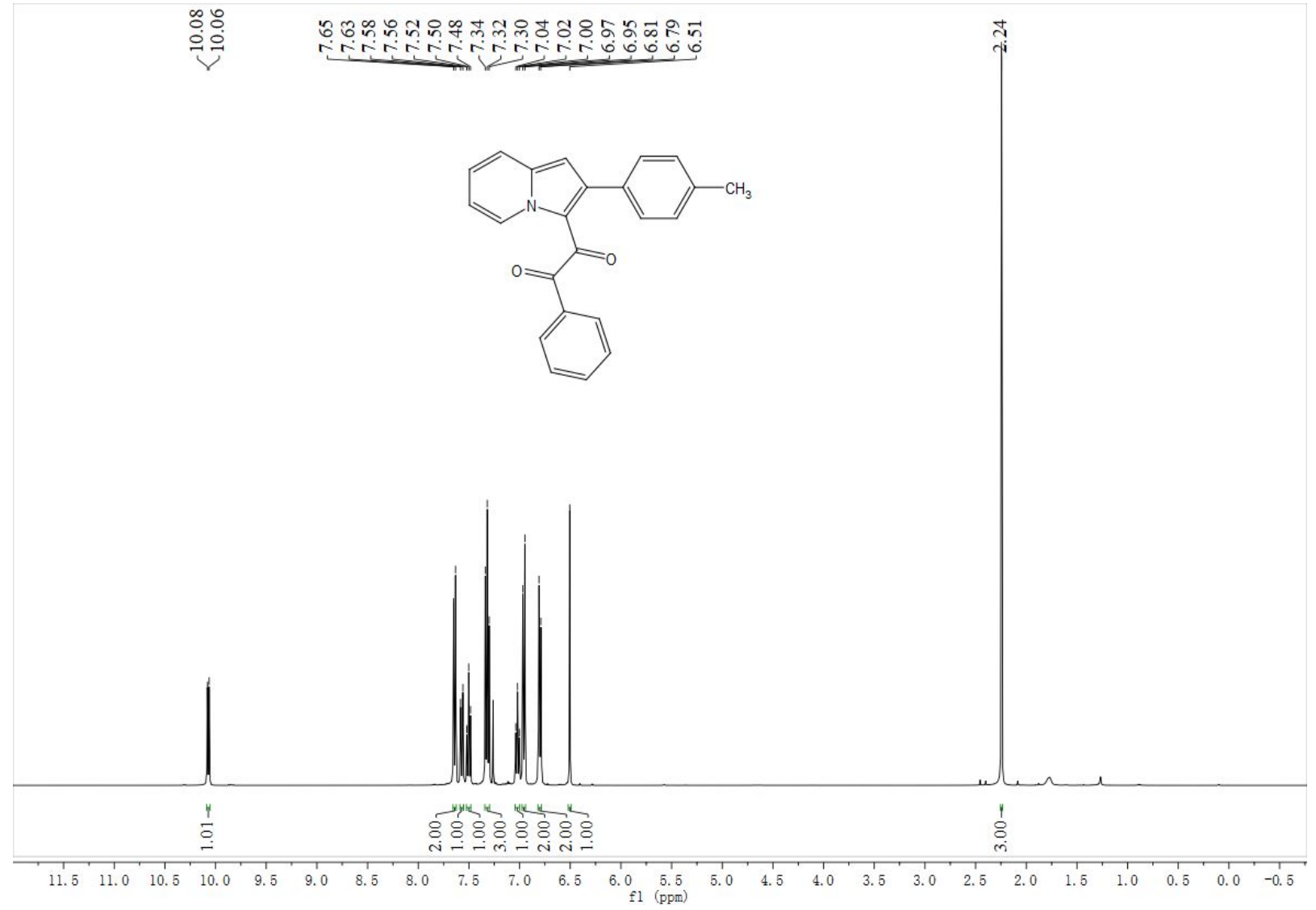

${ }^{13} \mathrm{C} \mathrm{NMR}\left(100 \mathrm{MHz}, \mathrm{CDCl}_{3}\right)$ spectrum of compound $\mathbf{3 e}$

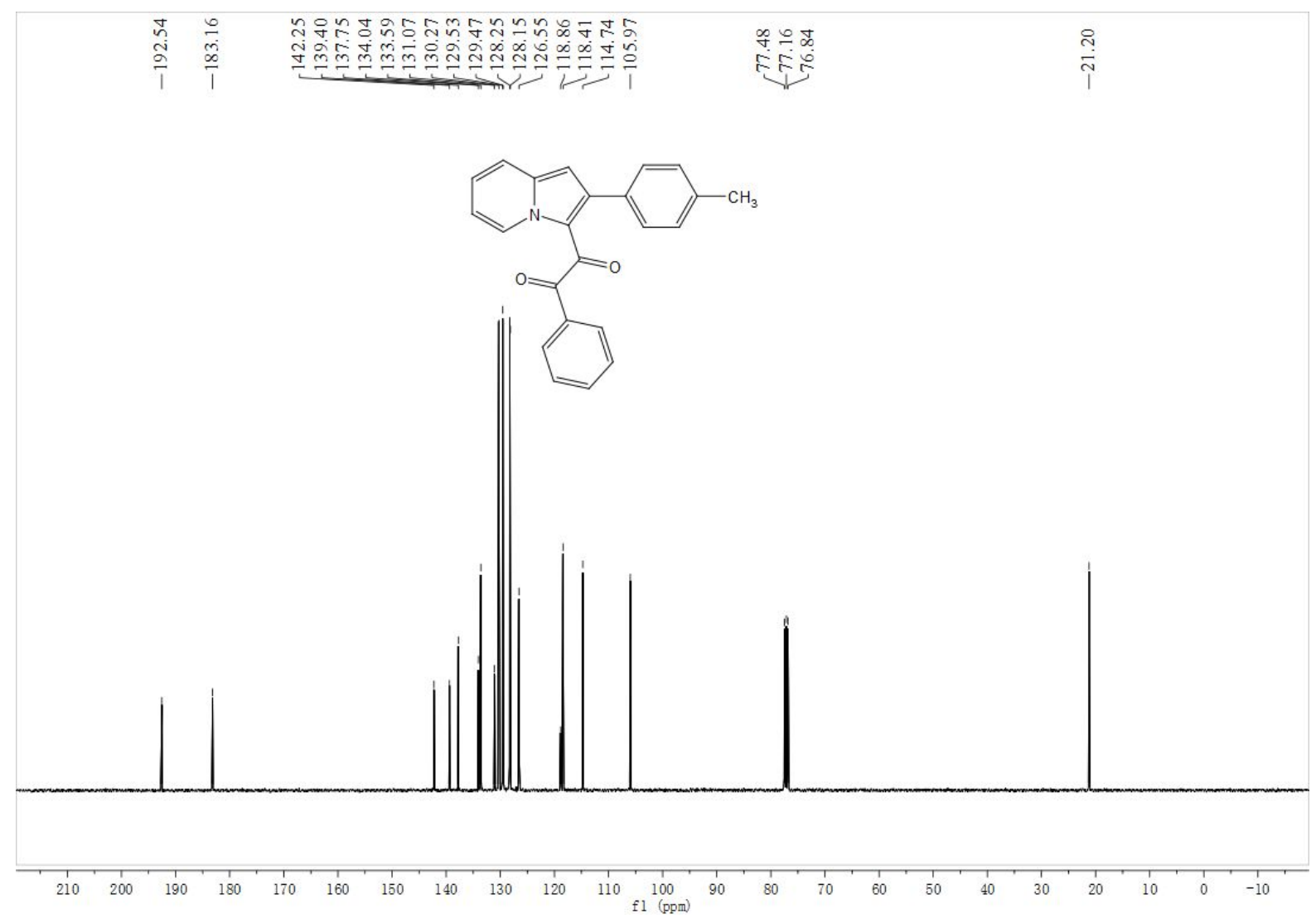


${ }^{1} \mathrm{H}$ NMR (400 MHz, $\mathrm{CDCl}_{3}$ ) spectrum of compound $\mathbf{3 f}$

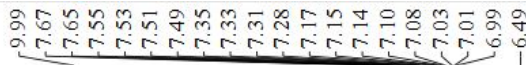
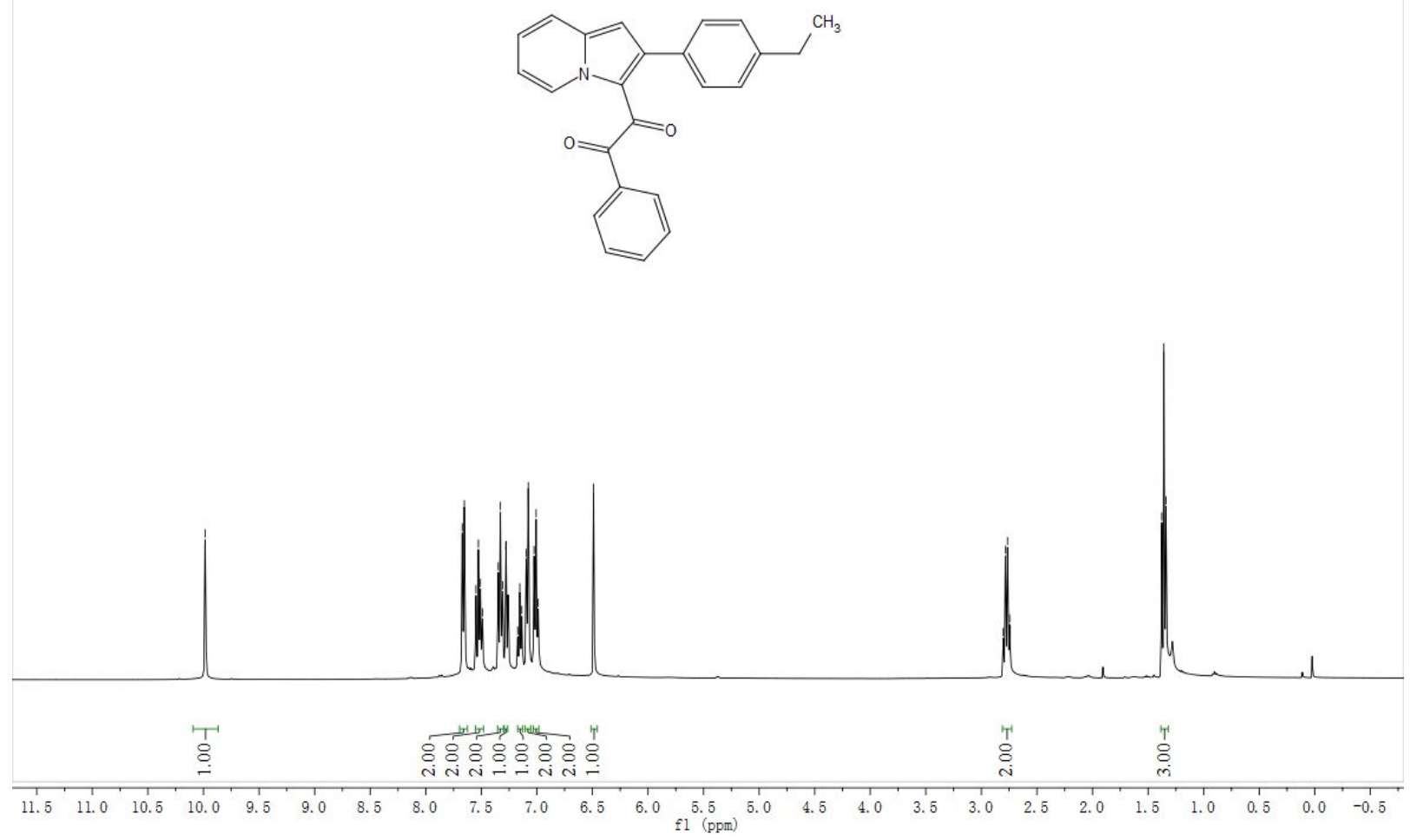

${ }^{13} \mathrm{C} \mathrm{NMR}\left(100 \mathrm{MHz}, \mathrm{CDCl}_{3}\right)$ spectrum of compound $\mathbf{3 f}$

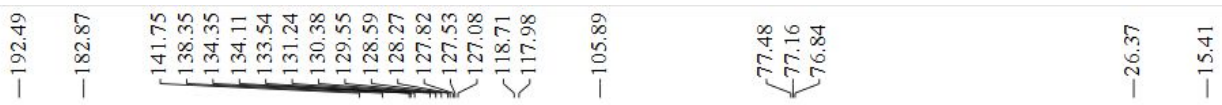

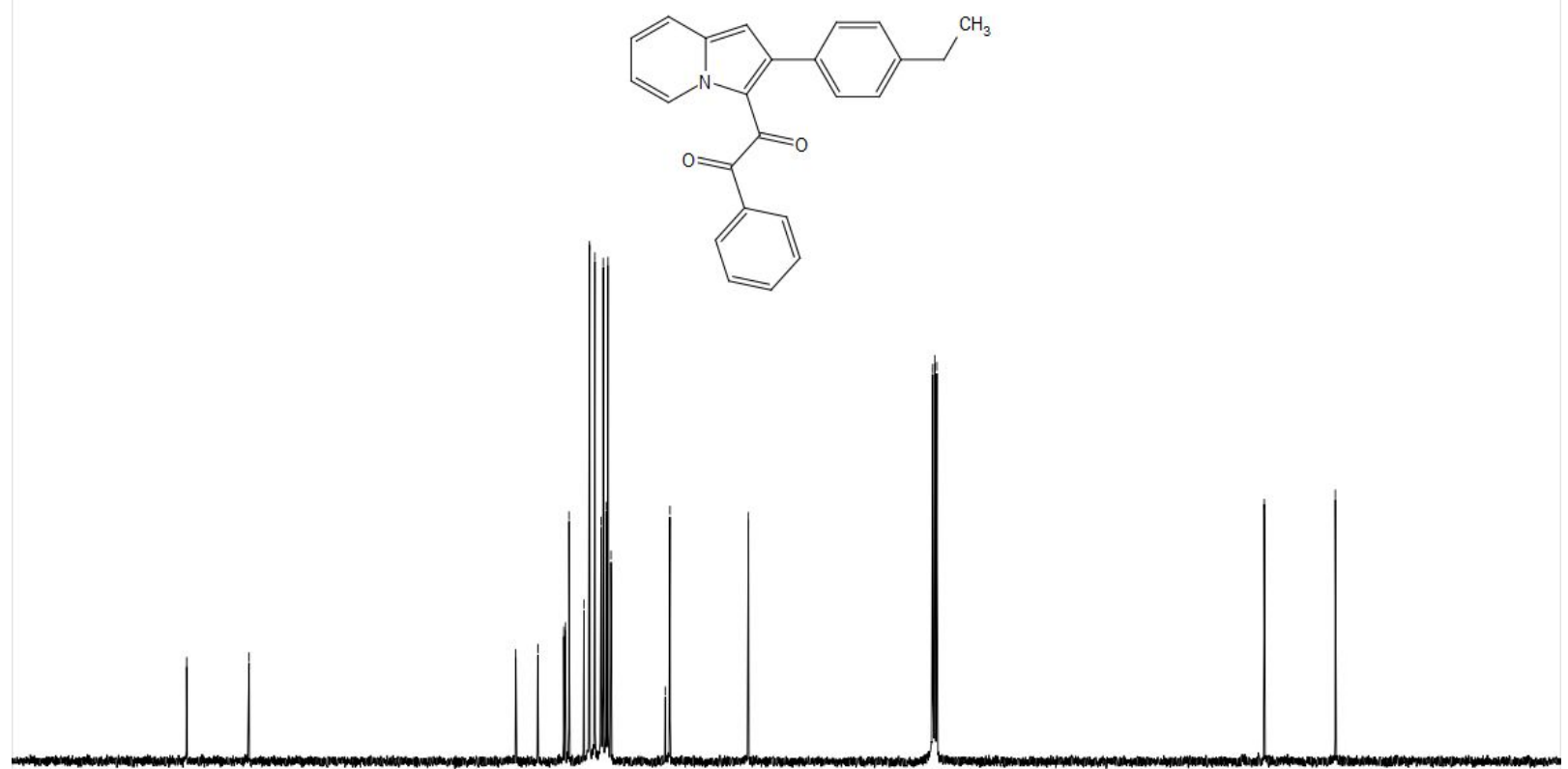

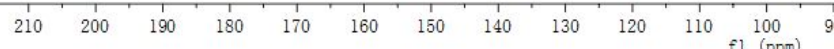


${ }^{1} \mathrm{H}$ NMR (400 MHz, $\mathrm{CDCl}_{3}$ ) spectrum of compound $\mathbf{3 g}$

웅우의

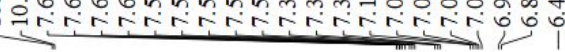
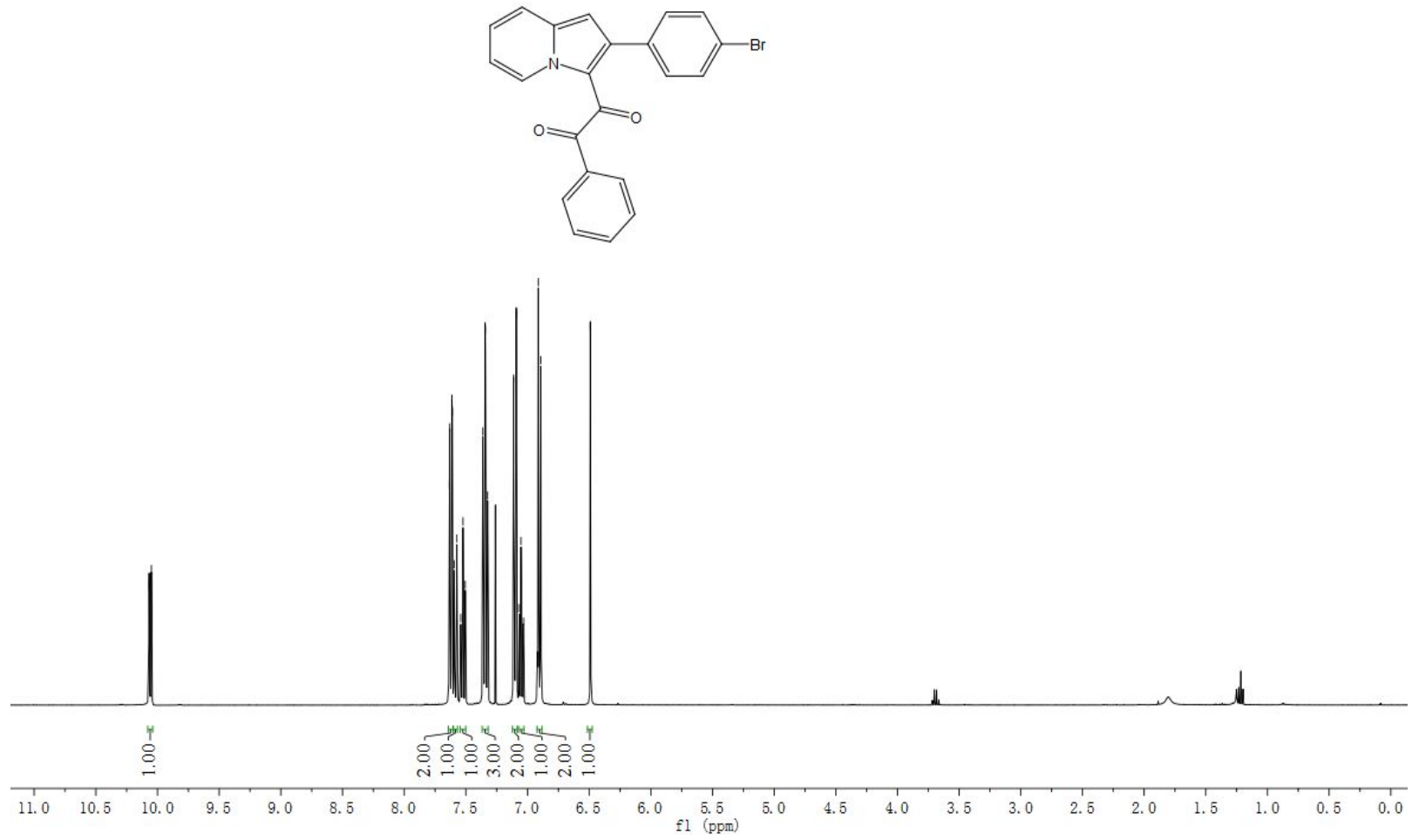

${ }^{13} \mathrm{C}$ NMR $\left(100 \mathrm{MHz}, \mathrm{CDCl}_{3}\right)$ spectrum of compound $\mathbf{3 g}$

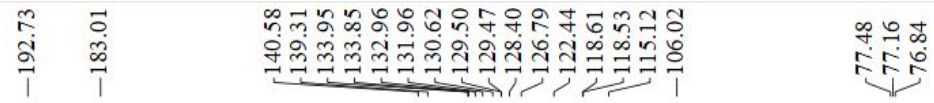

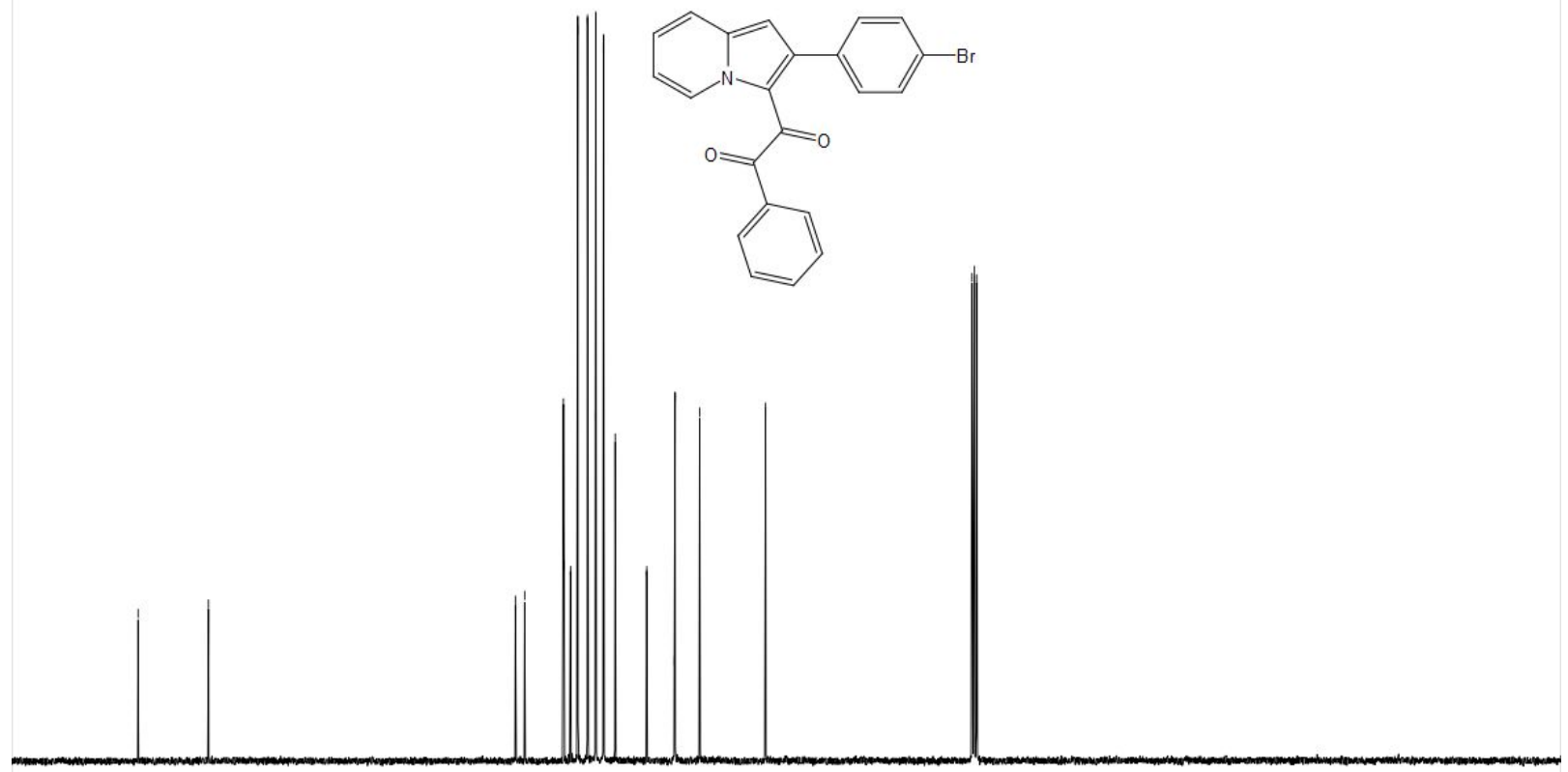

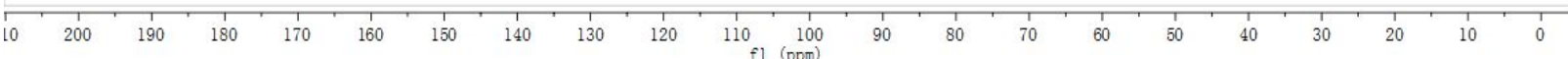


${ }^{1} \mathrm{H}$ NMR (400 MHz, $\mathrm{CDCl}_{3}$ ) spectrum of compound $\mathbf{3 h}$

年

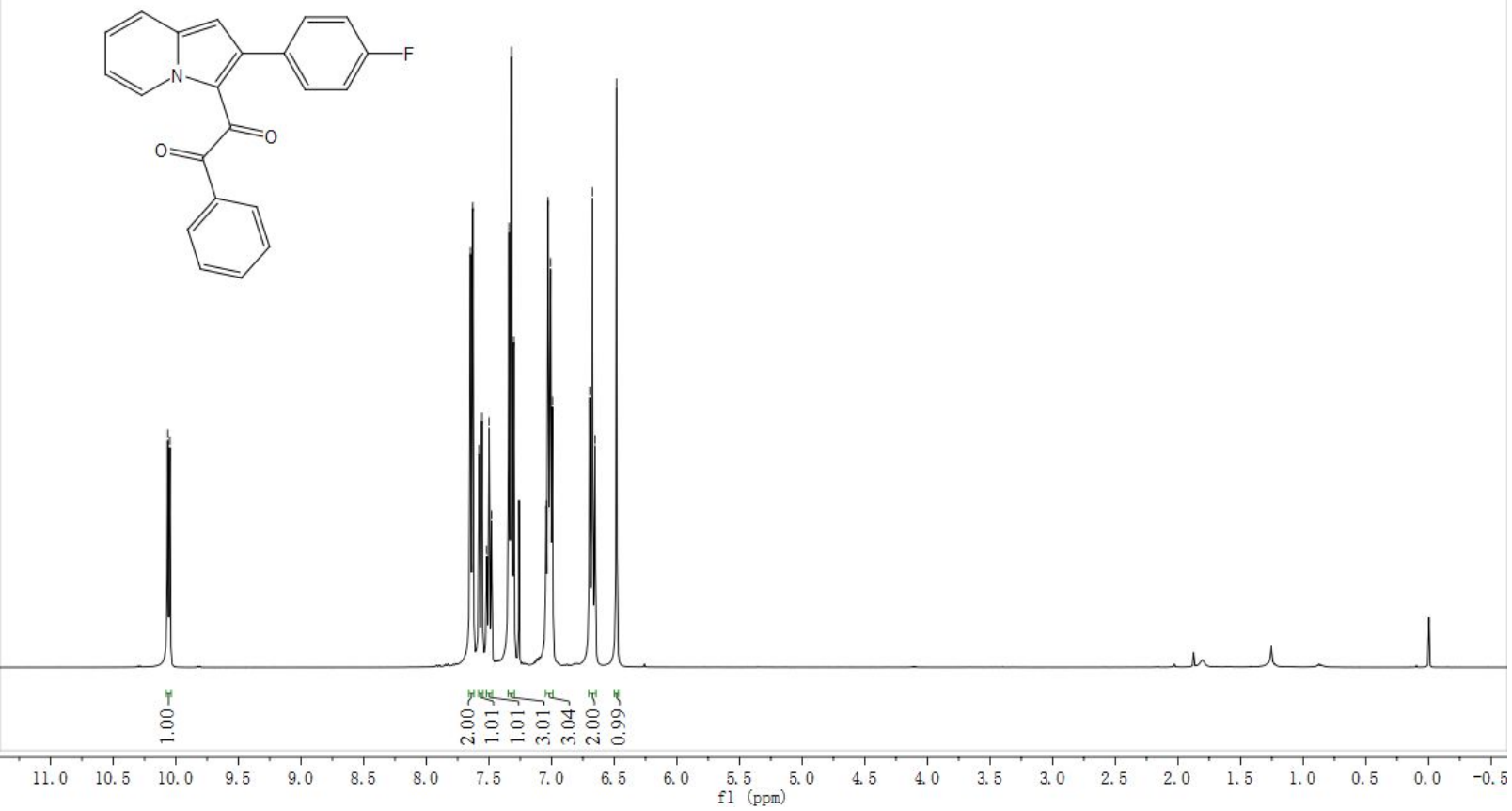

${ }^{13} \mathrm{C}$ NMR $\left(100 \mathrm{MHz}, \mathrm{CDCl}_{3}\right)$ spectrum of compound $\mathbf{3 h}$
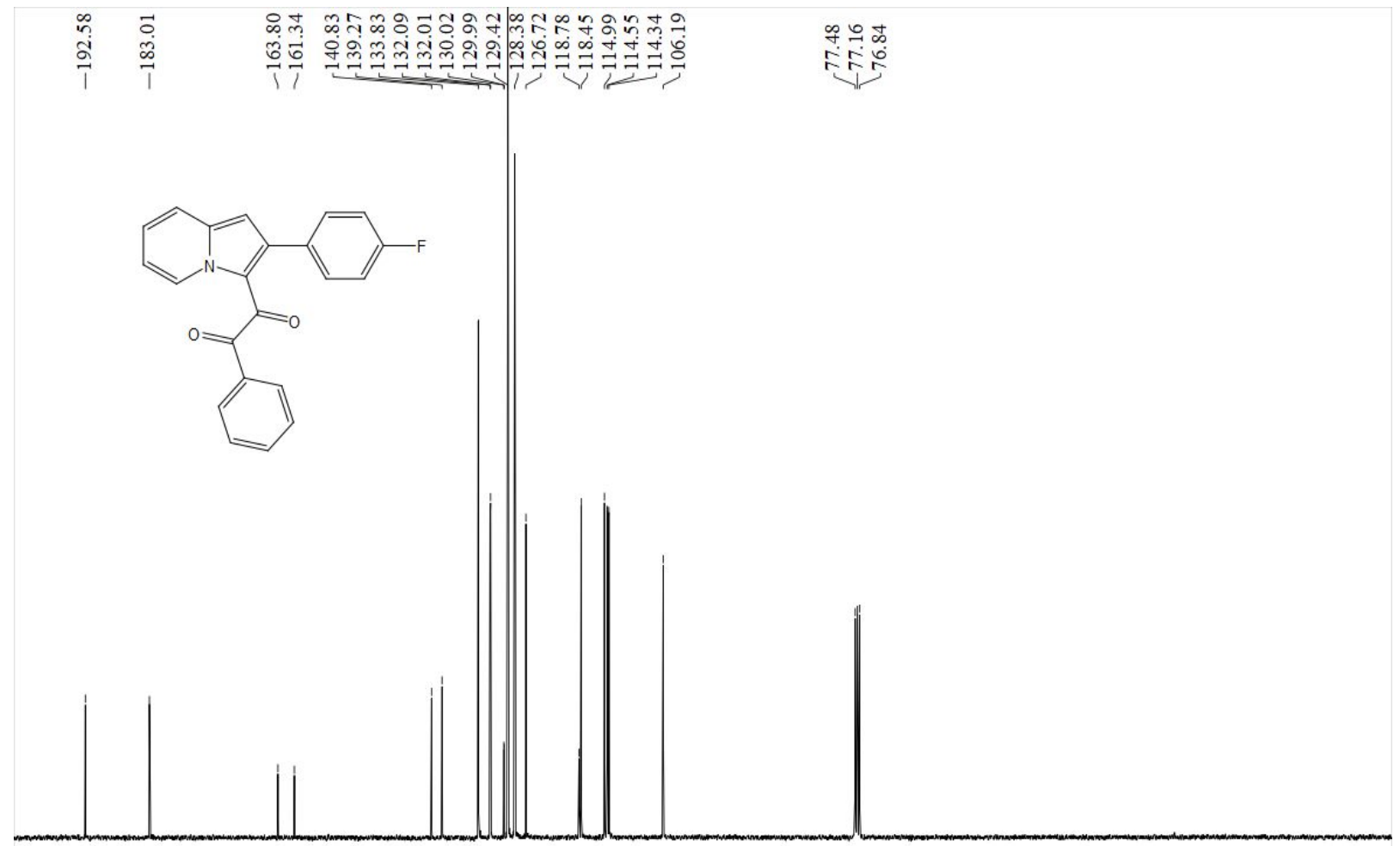

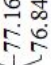

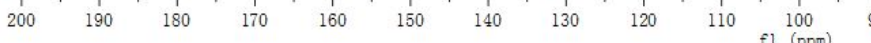


${ }^{1} \mathrm{H}$ NMR (400 MHz, $\mathrm{CDCl}_{3}$ ) spectrum of compound $\mathbf{3 i}$

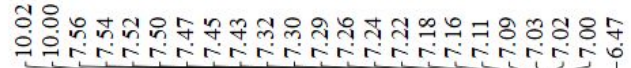
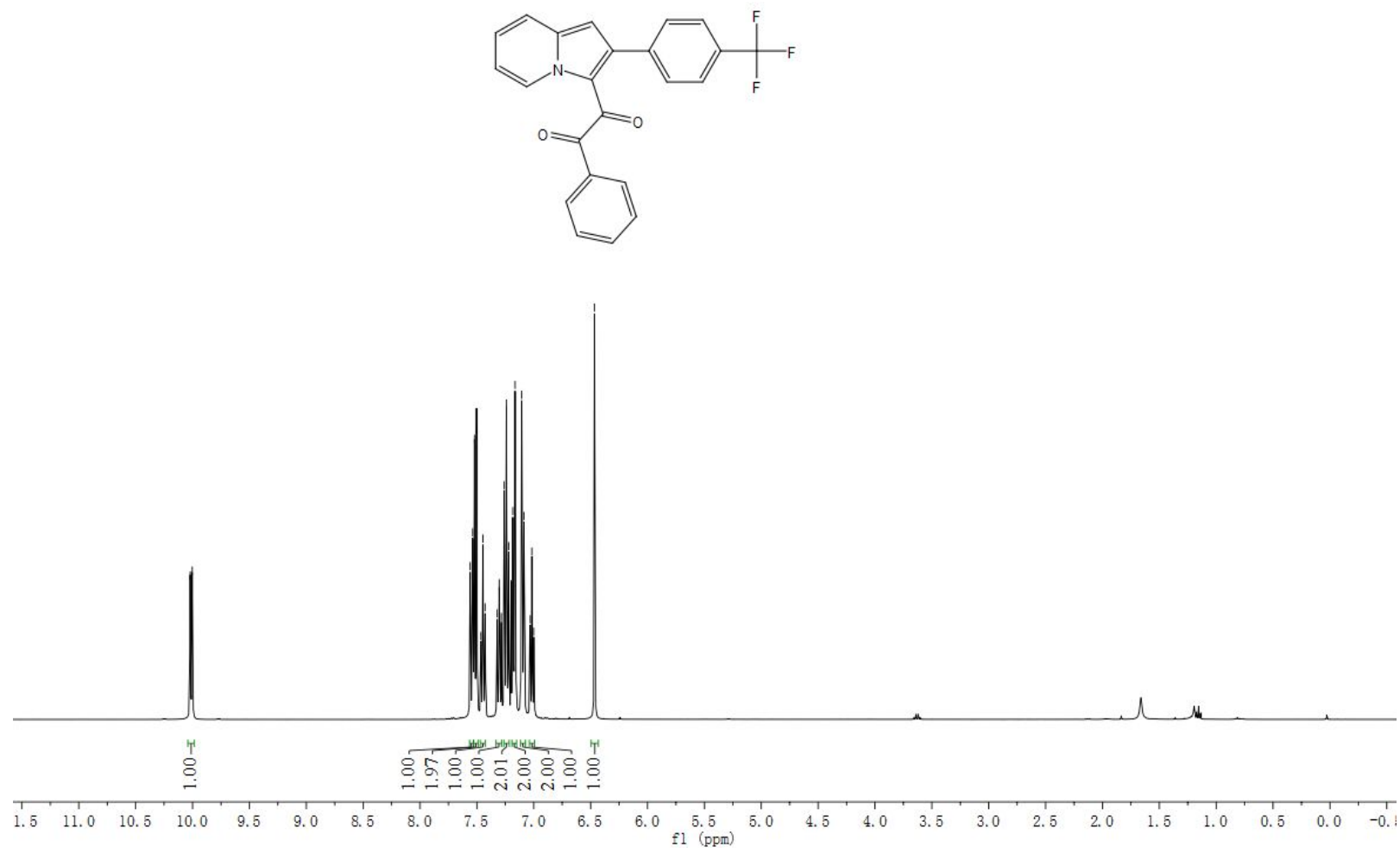

${ }^{13} \mathrm{C}$ NMR $\left(100 \mathrm{MHz}, \mathrm{CDCl}_{3}\right)$ spectrum of compound $\mathbf{3 i}$

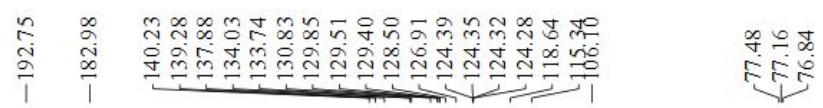

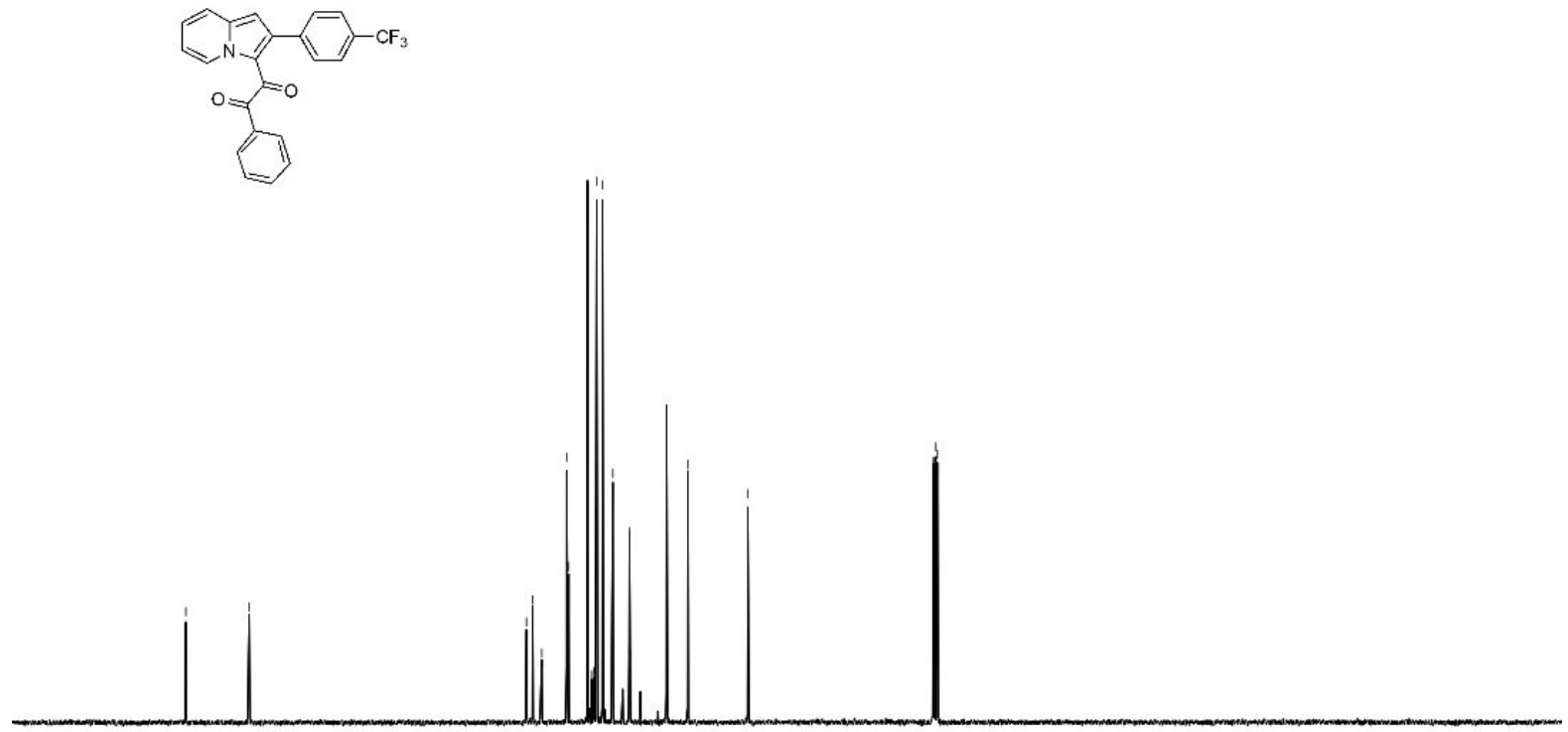

$\begin{array}{llllllllllll}210 & 200 & 190 & 180 & 170 & 160 & 150 & 140 & 130 & 120 & 110 & 100 \\ \mathrm{f} 1(\mathrm{ppm}) & 90\end{array}$ 
${ }^{1} \mathrm{H}$ NMR (400 MHz, $\left.\mathrm{CDCl}_{3}\right)$ spectrum of compound $\mathbf{3 j}$

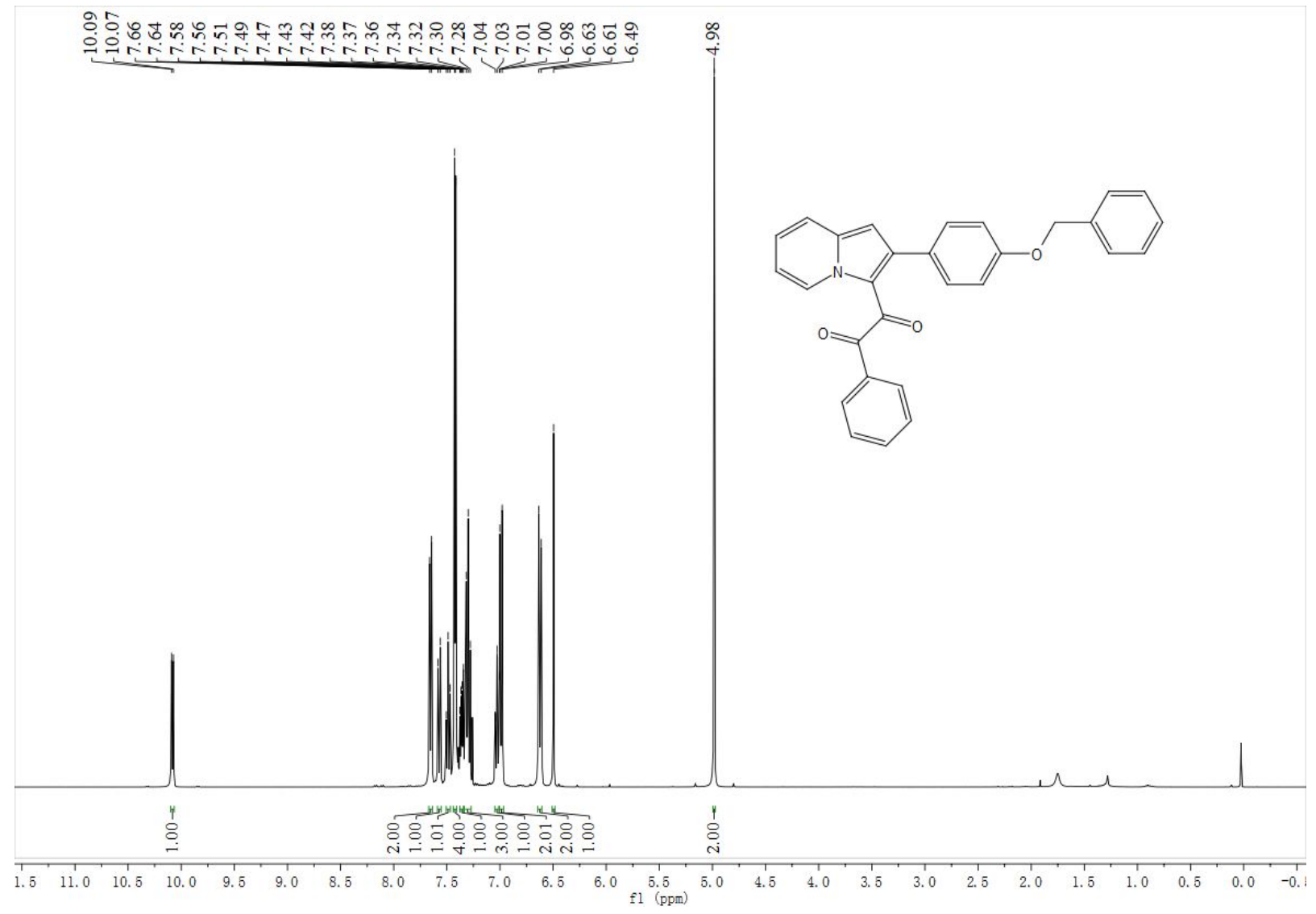

${ }^{13} \mathrm{C} \mathrm{NMR}\left(100 \mathrm{MHz}, \mathrm{CDCl}_{3}\right)$ spectrum of compound $\mathbf{3 j}$

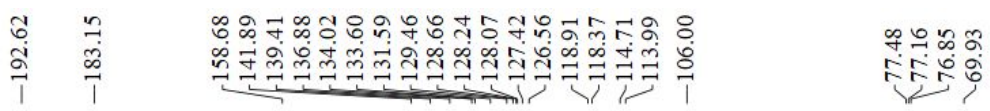

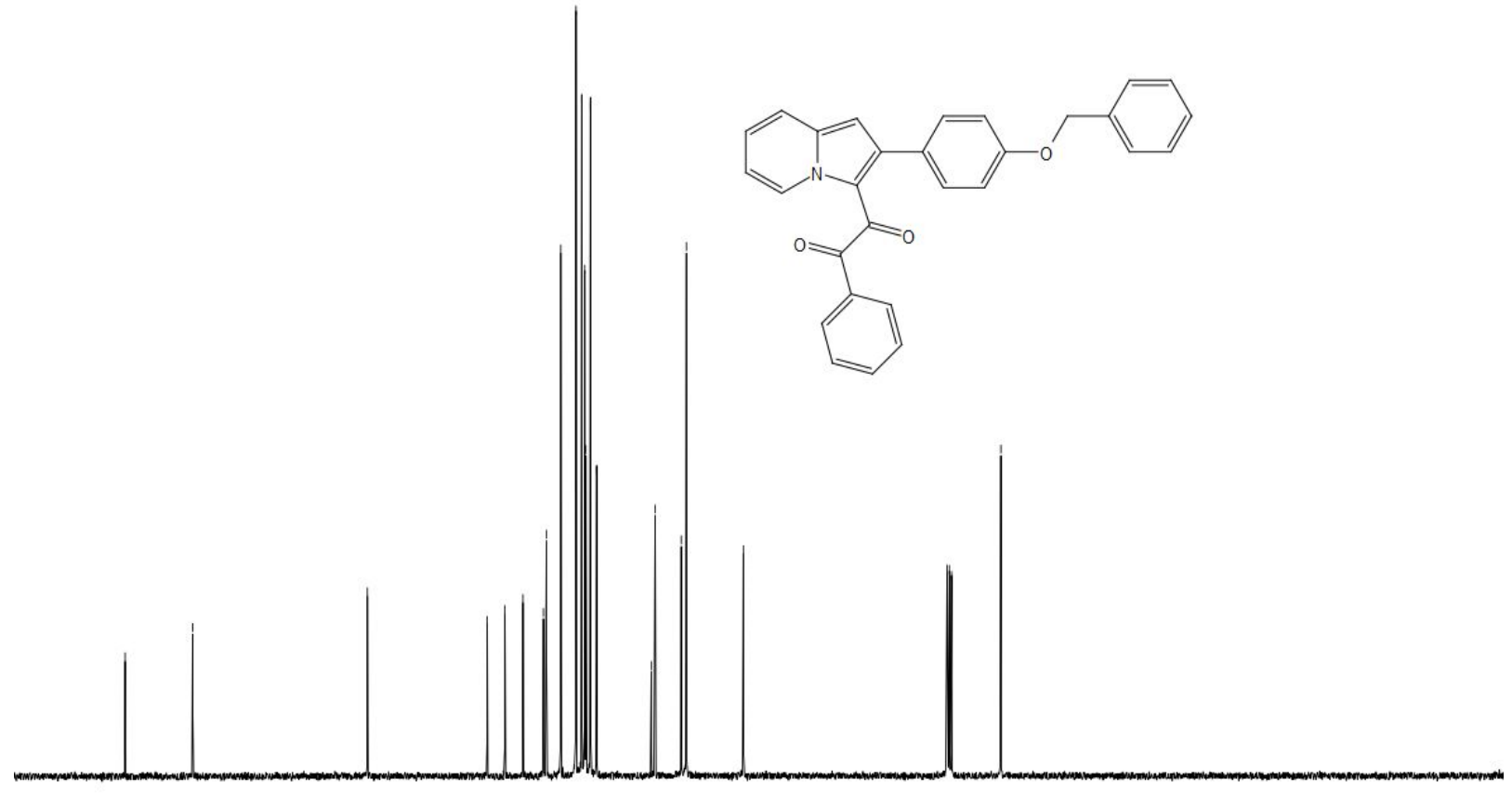

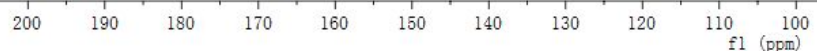


${ }^{1} \mathrm{H}$ NMR (400 MHz, $\mathrm{CDCl}_{3}$ ) spectrum of compound $\mathbf{3 k}$

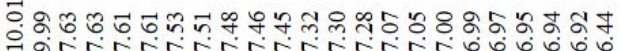

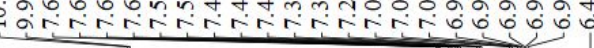

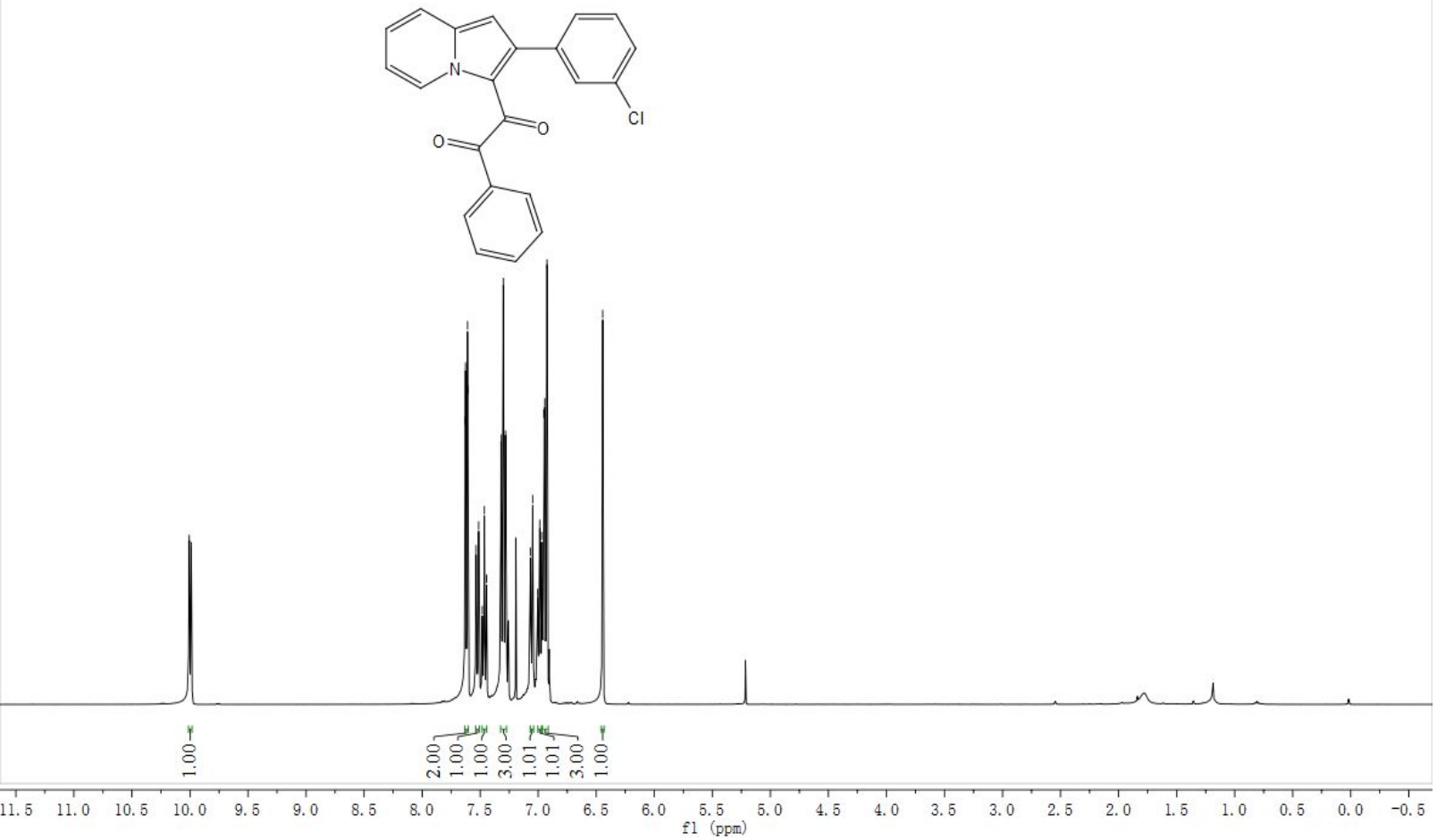

${ }^{13} \mathrm{C}$ NMR $\left(100 \mathrm{MHz}, \mathrm{CDCl}_{3}\right)$ spectrum of compound 3k

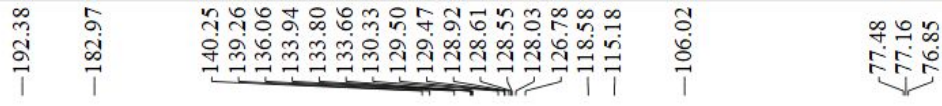

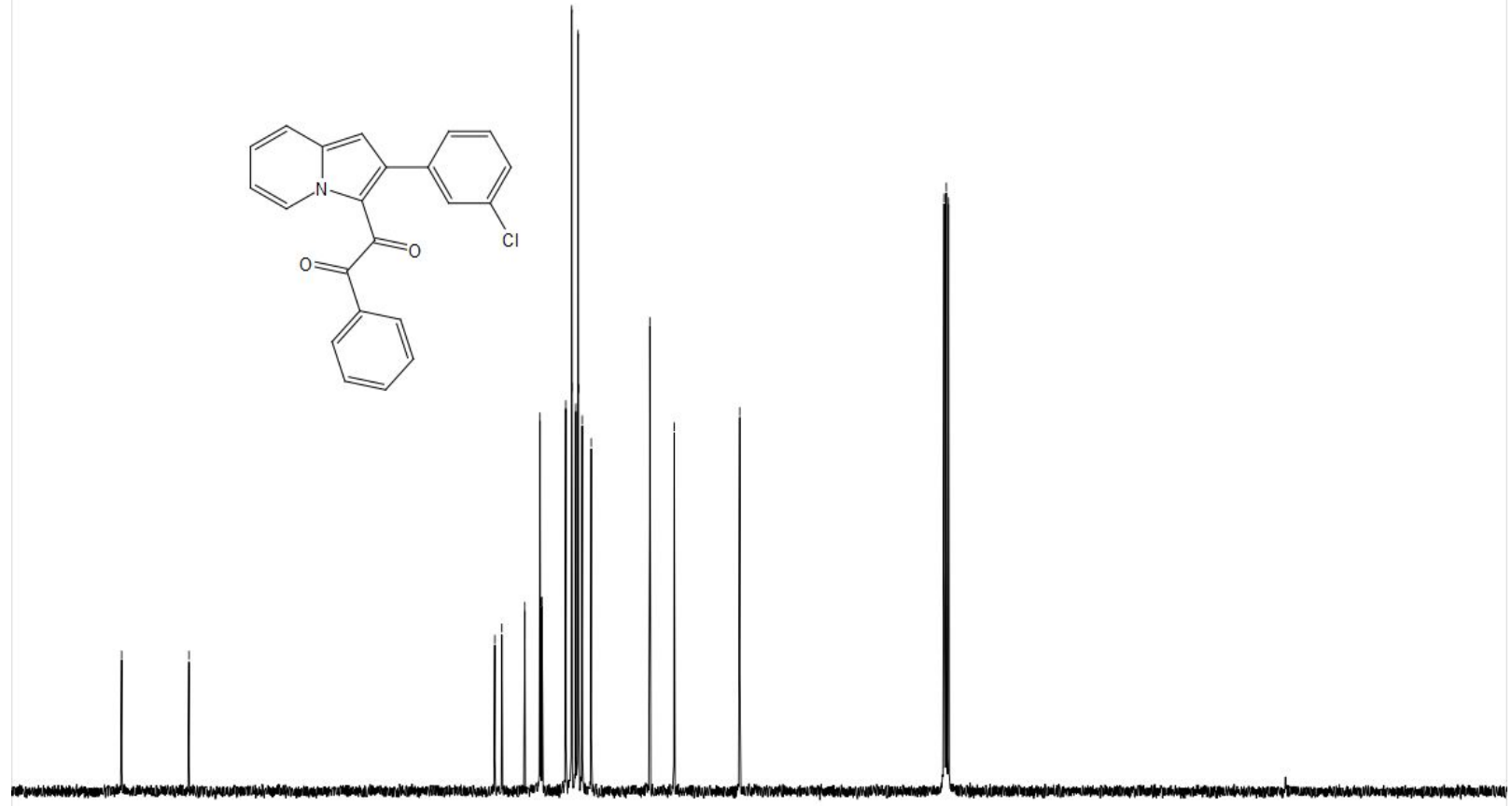

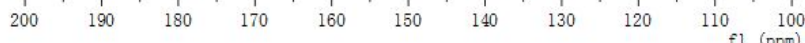


${ }^{1} \mathrm{H}$ NMR (400 MHz, $\mathrm{CDCl}_{3}$ ) spectrum of compound $3 \mathbf{I}$

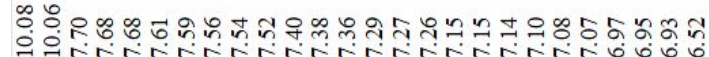

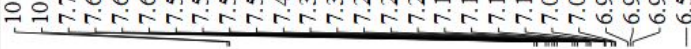

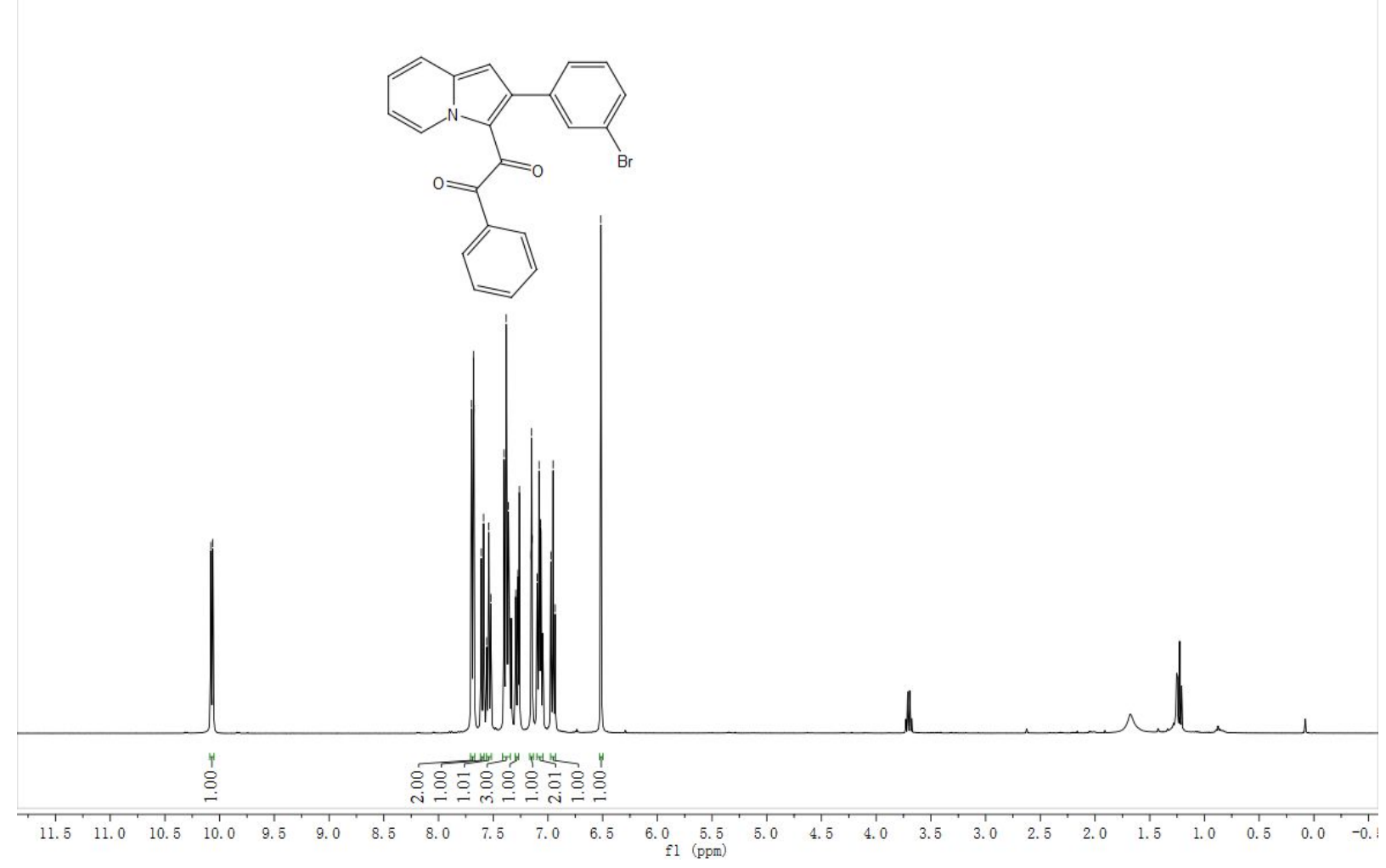

${ }^{13} \mathrm{C}$ NMR (100 MHz, $\left.\mathrm{CDCl}_{3}\right)$ spectrum of compound $3 \mathbf{I}$

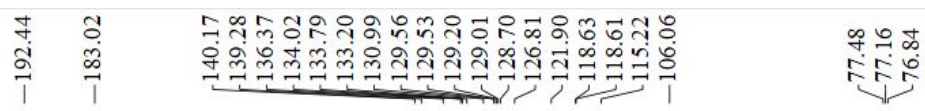

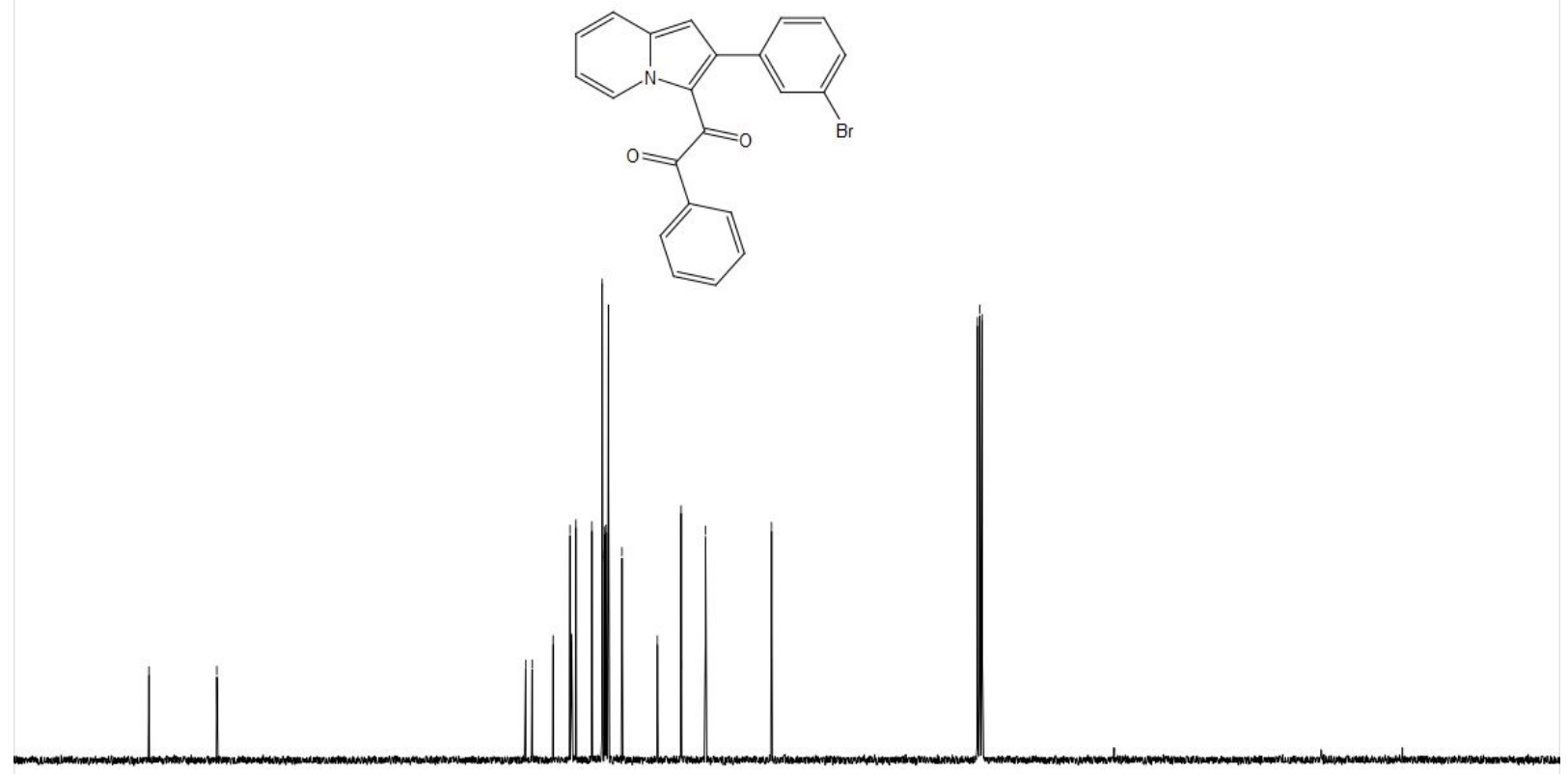

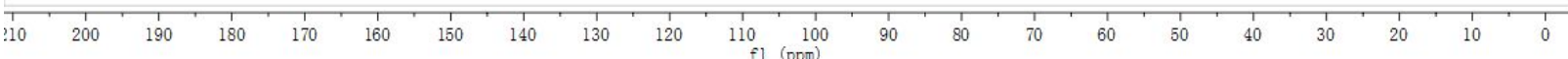


${ }^{1} \mathrm{H}$ NMR (400 MHz, $\mathrm{CDCl}_{3}$ ) spectrum of compound 3m

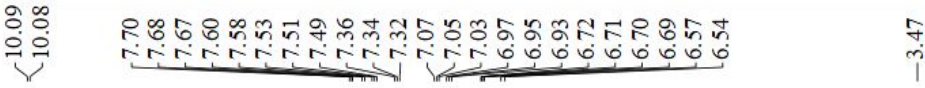<smiles>COc1cccc(-c2cc3ccccn3c2C(=O)C(=O)c2ccccc2)c1</smiles>

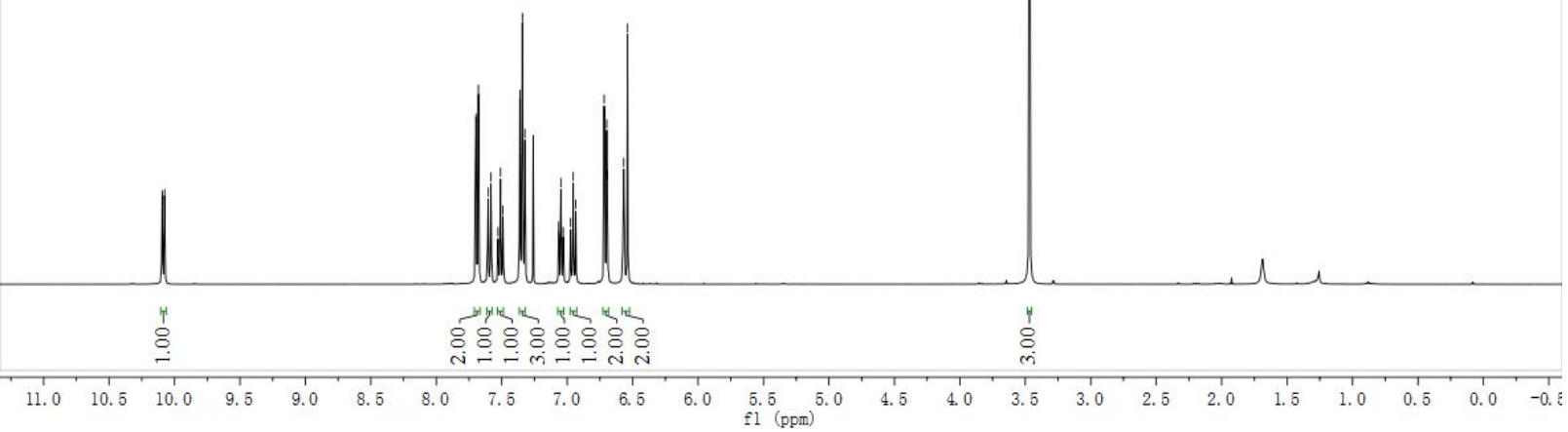

${ }^{13} \mathrm{C} \mathrm{NMR}\left(100 \mathrm{MHz}, \mathrm{CDCl}_{3}\right)$ spectrum of compound $\mathbf{3 m}$

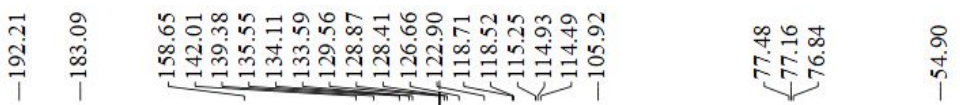<smiles>COc1cccc(-c2cc3ccccn3c2C(=O)C(=O)c2ccccc2)c1</smiles> 
${ }^{1} \mathrm{H}$ NMR (400 MHz, $\mathrm{CDCl}_{3}$ ) spectrum of compound 3n

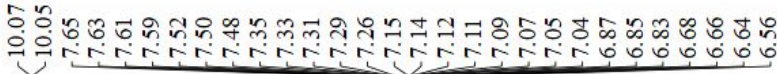

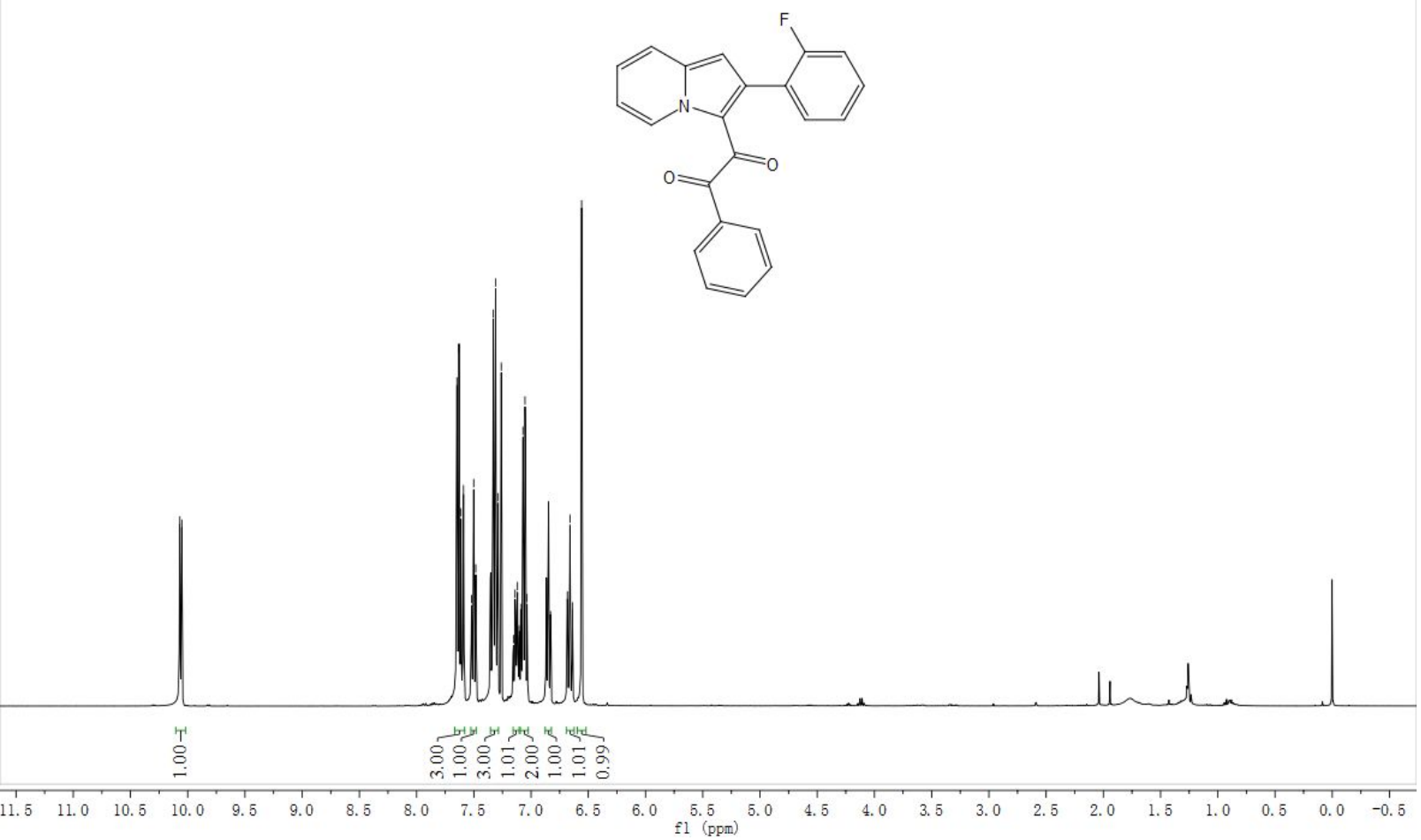

${ }^{13} \mathrm{C}$ NMR $\left(100 \mathrm{MHz}, \mathrm{CDCl}_{3}\right)$ spectrum of compound 3n

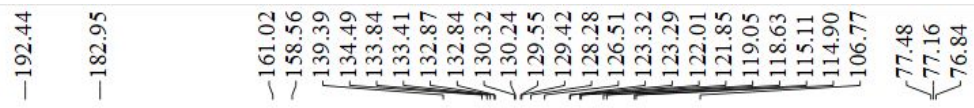
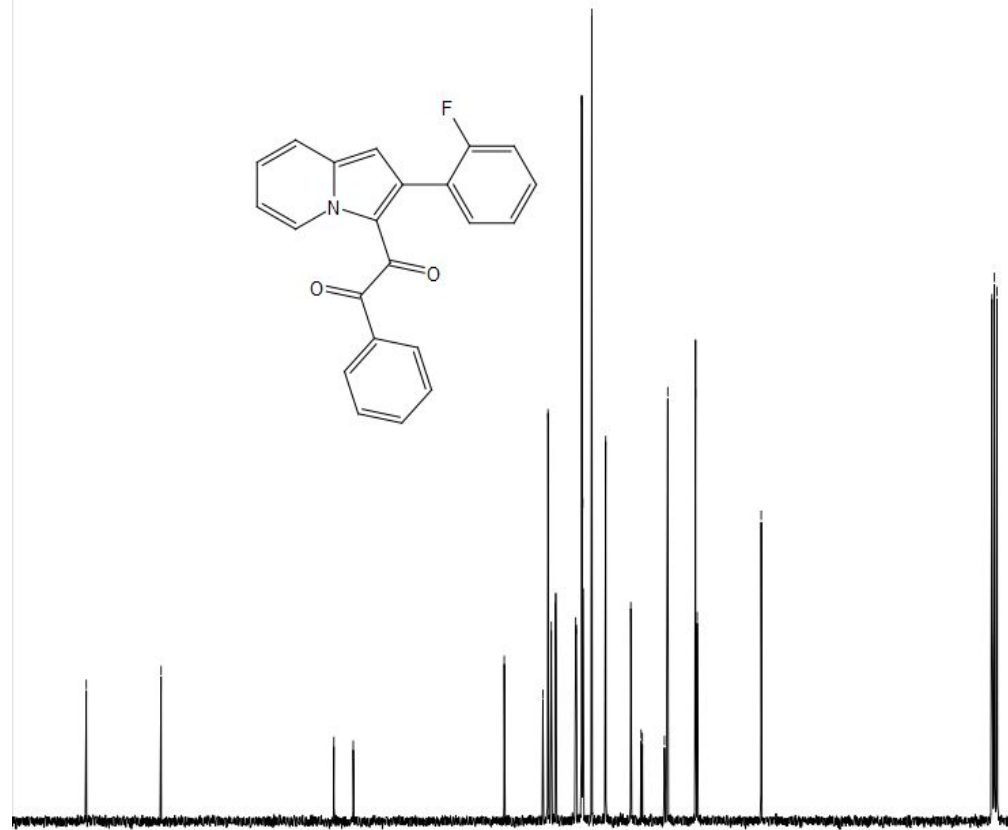

200

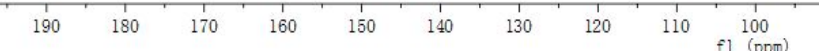


${ }^{1} \mathrm{H}$ NMR (400 MHz, $\mathrm{CDCl}_{3}$ ) spectrum of compound 3o

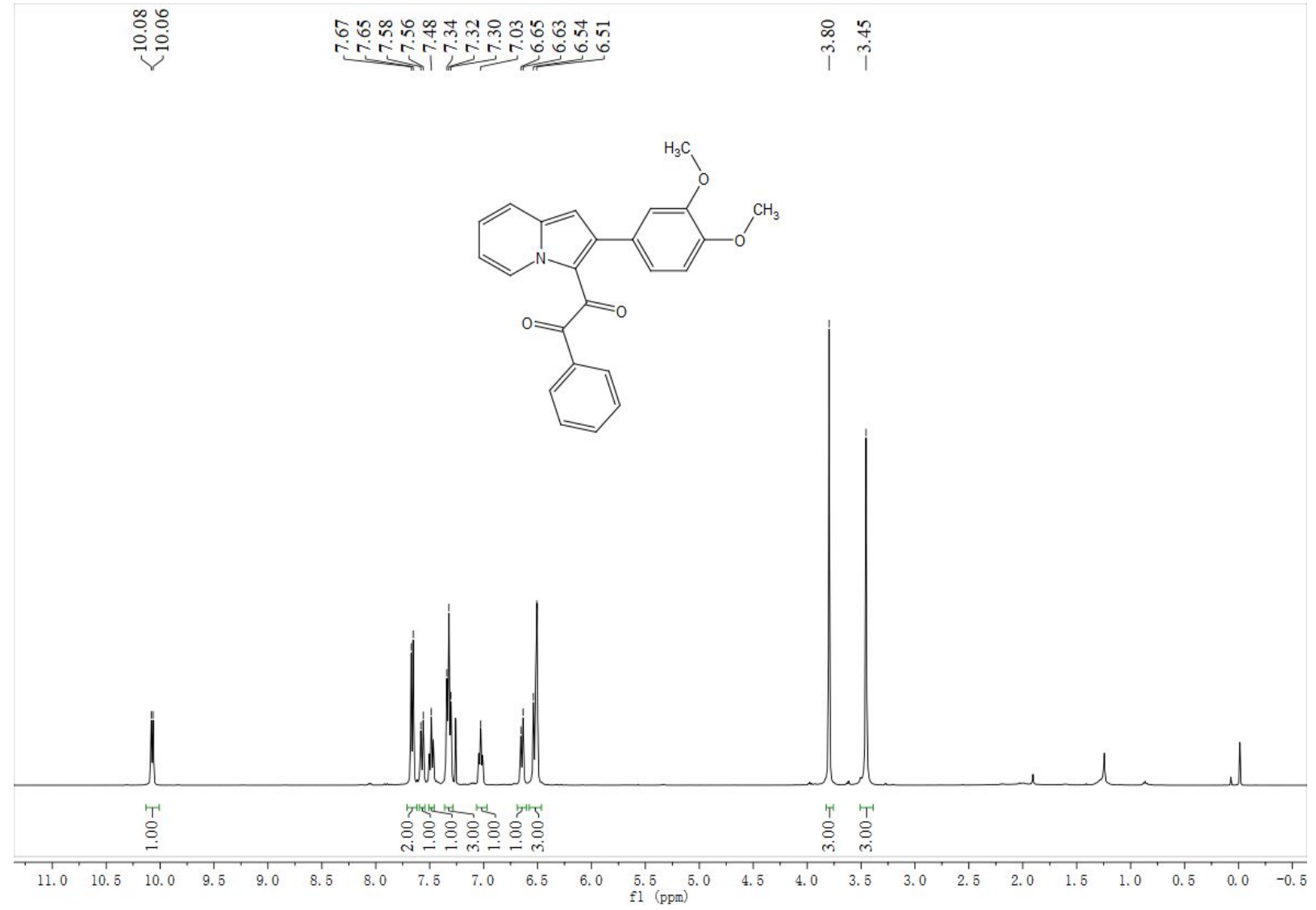

${ }^{13} \mathrm{C}$ NMR $\left(100 \mathrm{MHz}, \mathrm{CDCl}_{3}\right)$ spectrum of compound 30

\begin{tabular}{|c|c|c|}
\hline 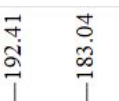 & 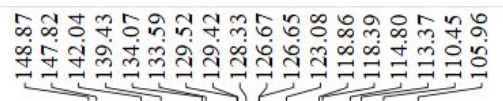 & 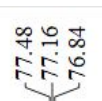 \\
\hline
\end{tabular}
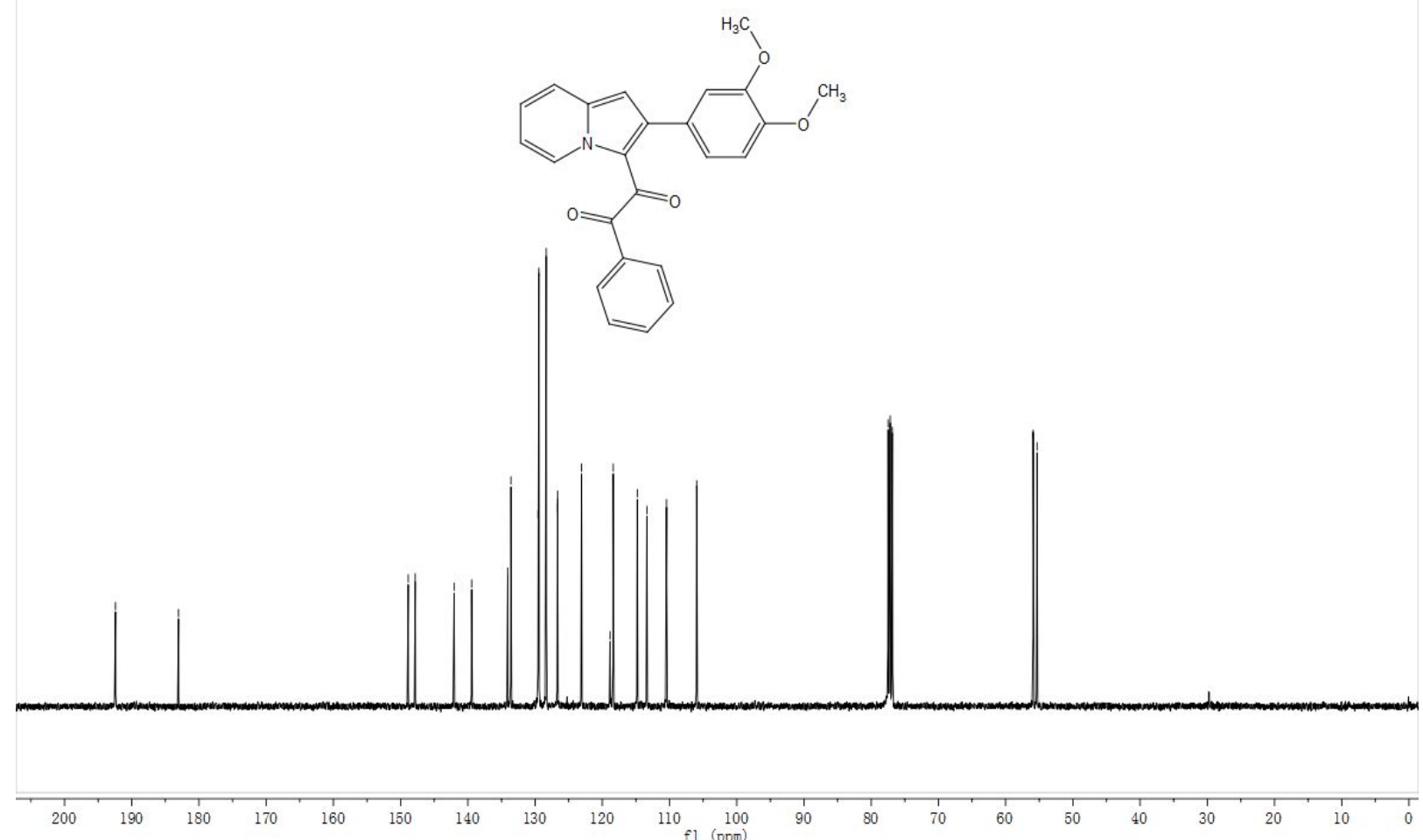
${ }^{1} \mathrm{H}$ NMR (400 MHz, $\mathrm{CDCl}_{3}$ ) spectrum of compound 3p

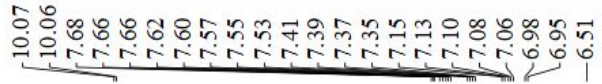
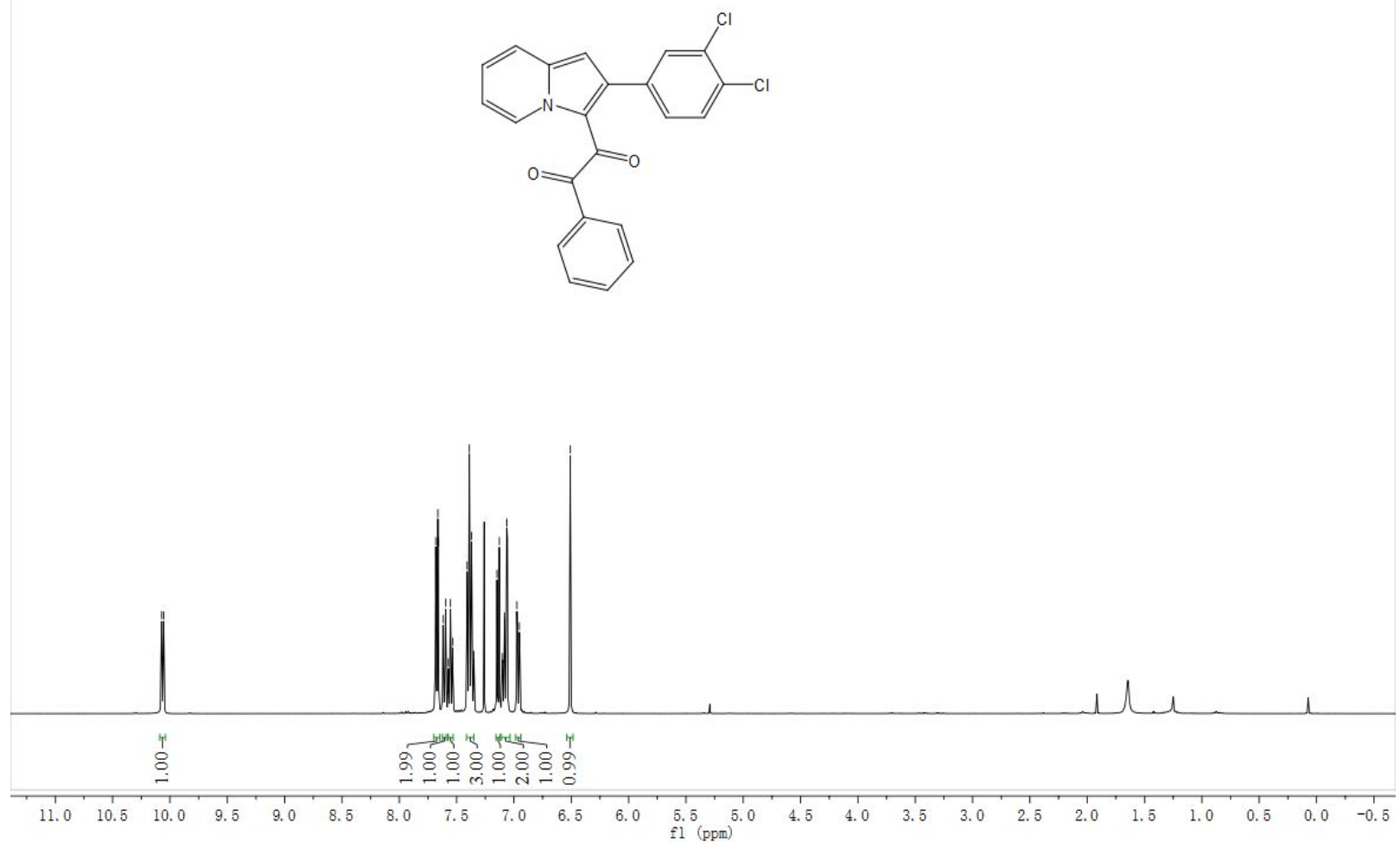

${ }^{13} \mathrm{C} \mathrm{NMR}\left(100 \mathrm{MHz}, \mathrm{CDCl}_{3}\right)$ spectrum of compound 3p

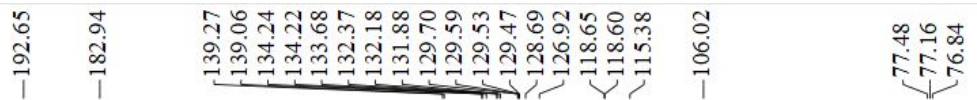
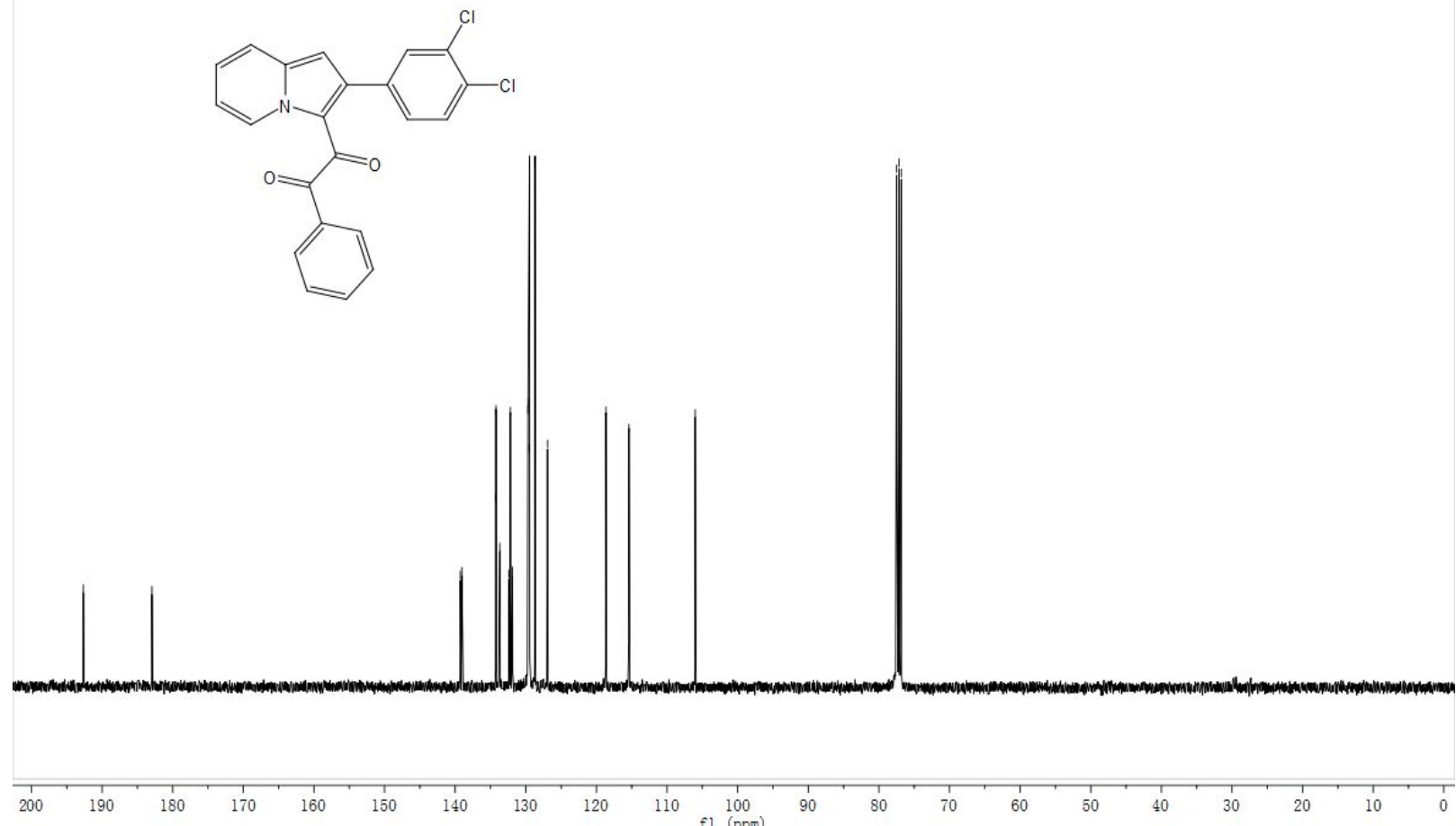
${ }^{1} \mathrm{H}$ NMR (400 MHz, $\mathrm{CDCl}_{3}$ ) spectrum of compound $\mathbf{3 q}$

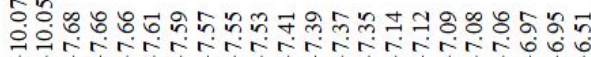
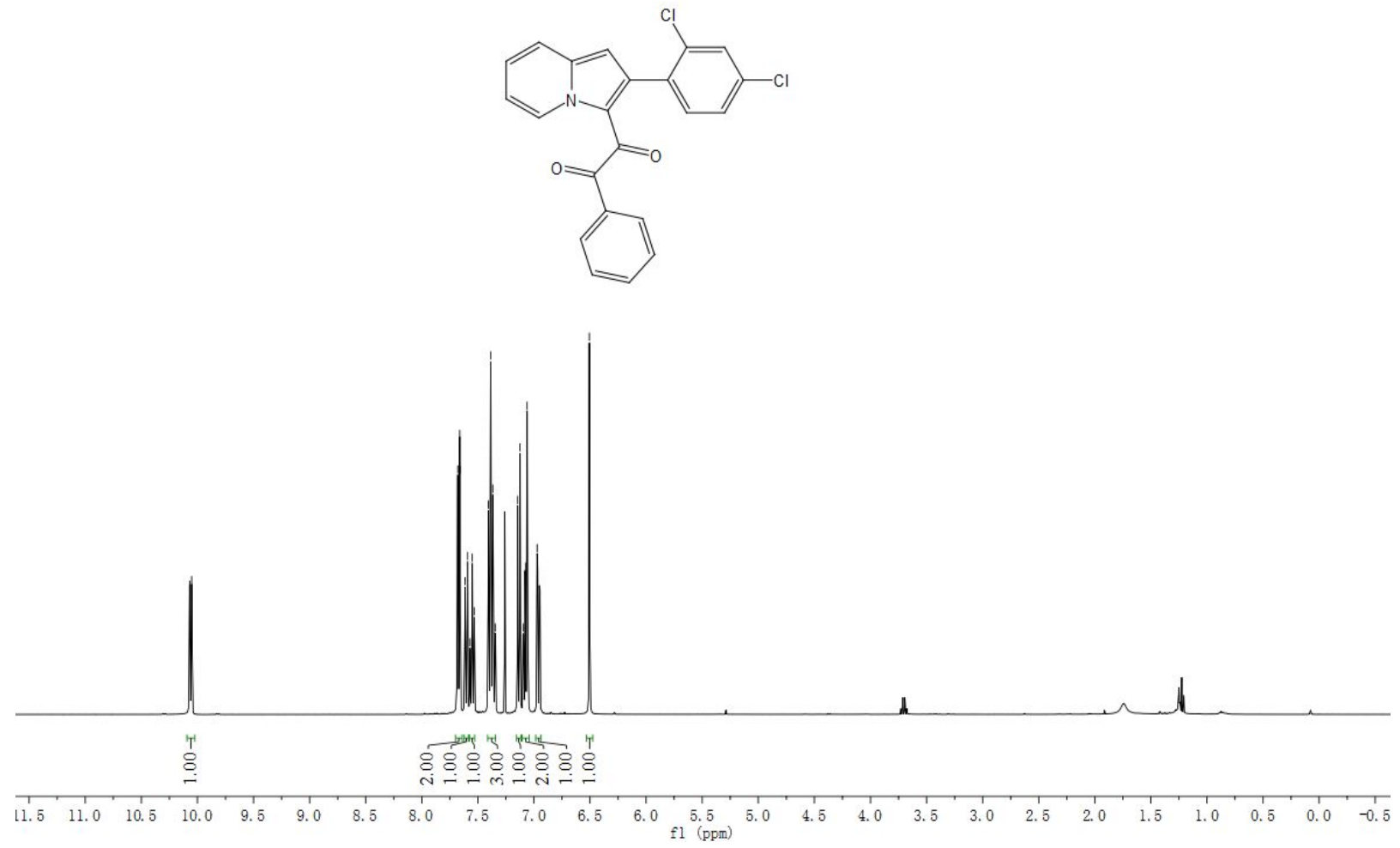

${ }^{13} \mathrm{C}$ NMR $\left(100 \mathrm{MHz}, \mathrm{CDCl}_{3}\right)$ spectrum of compound $\mathbf{3 q}$

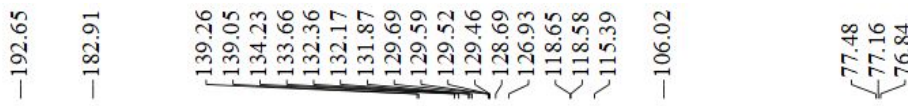
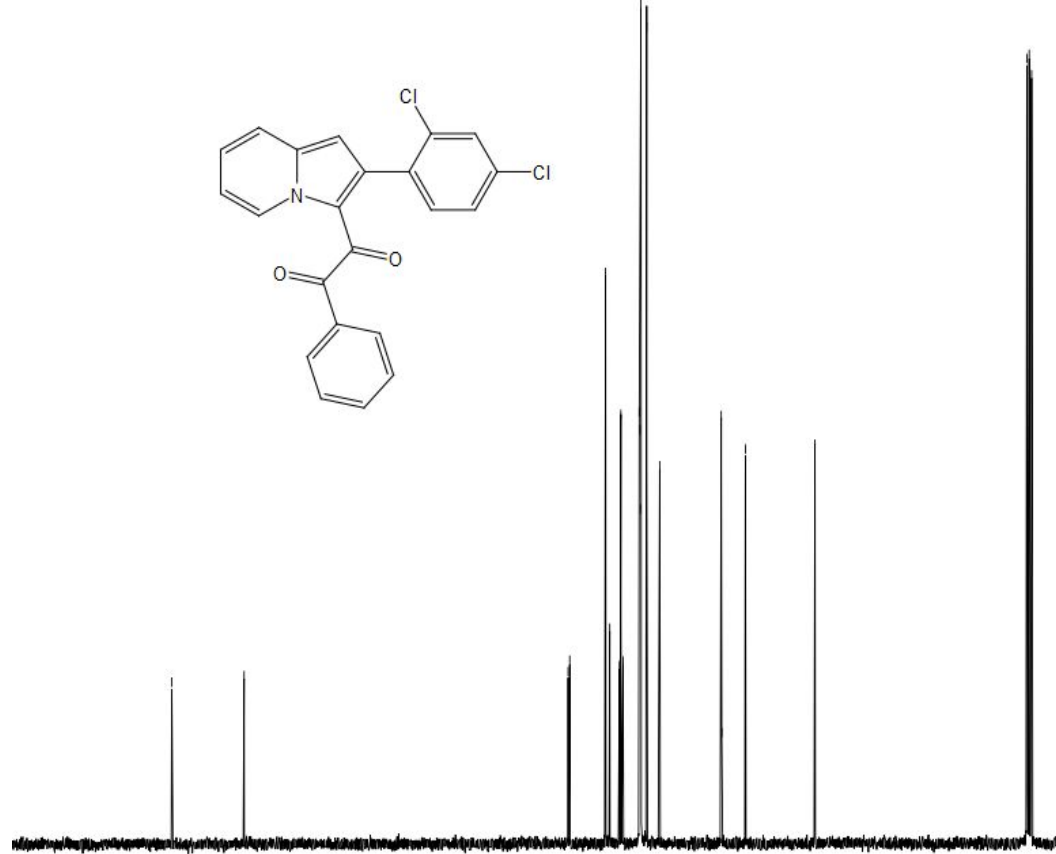

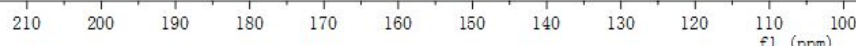


${ }^{1} \mathrm{H}$ NMR (400 MHz, $\mathrm{CDCl}_{3}$ ) spectrum of compound 3r

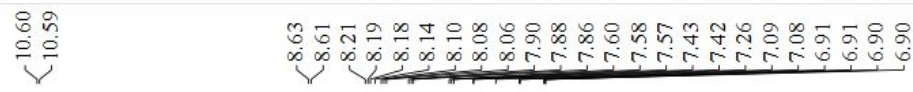
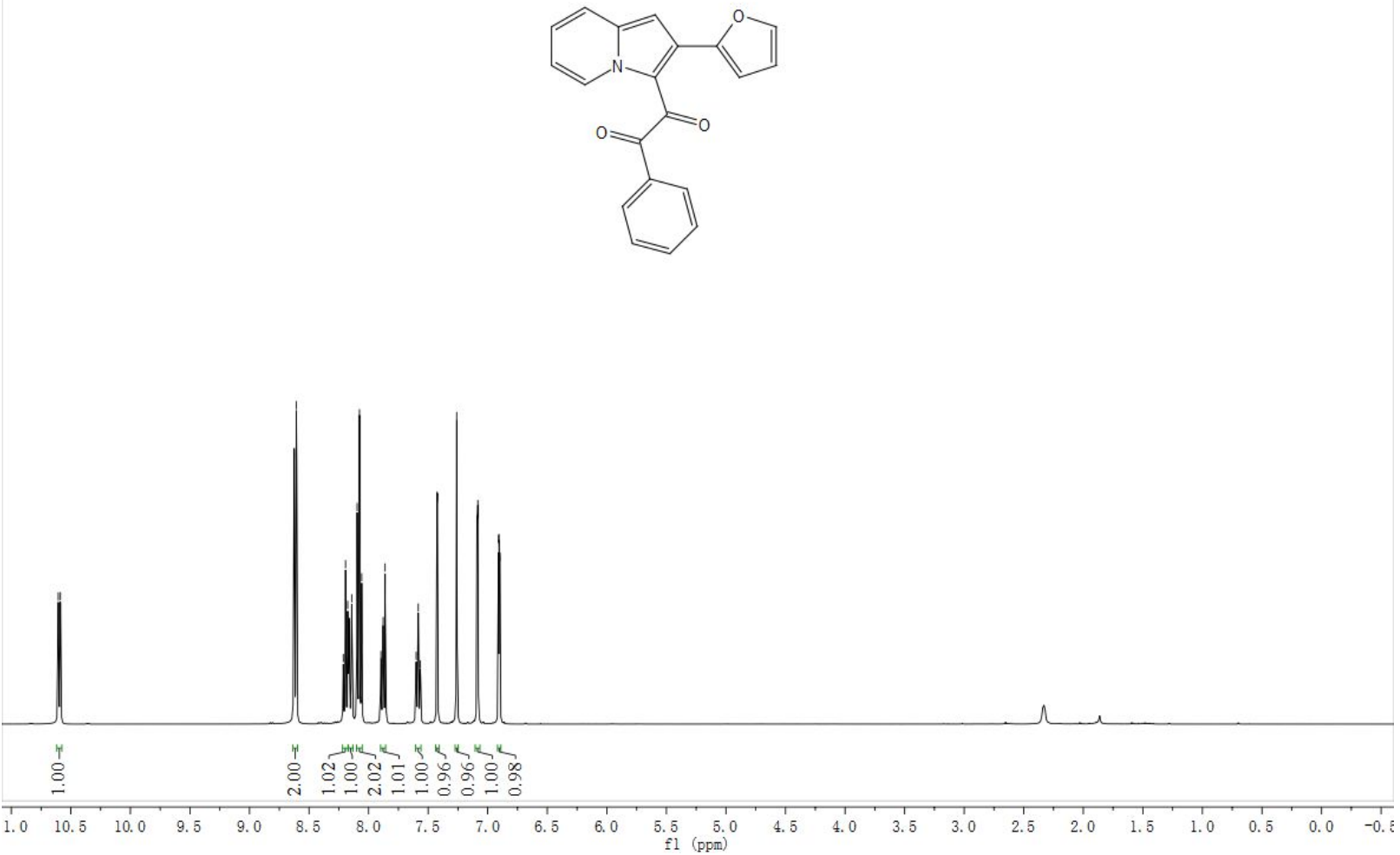

${ }^{13} \mathrm{C} \mathrm{NMR}\left(100 \mathrm{MHz}, \mathrm{CDCl}_{3}\right)$ spectrum of compound $3 \mathbf{r}$

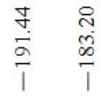

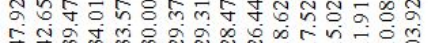

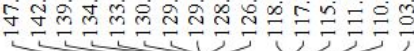

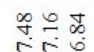
笛
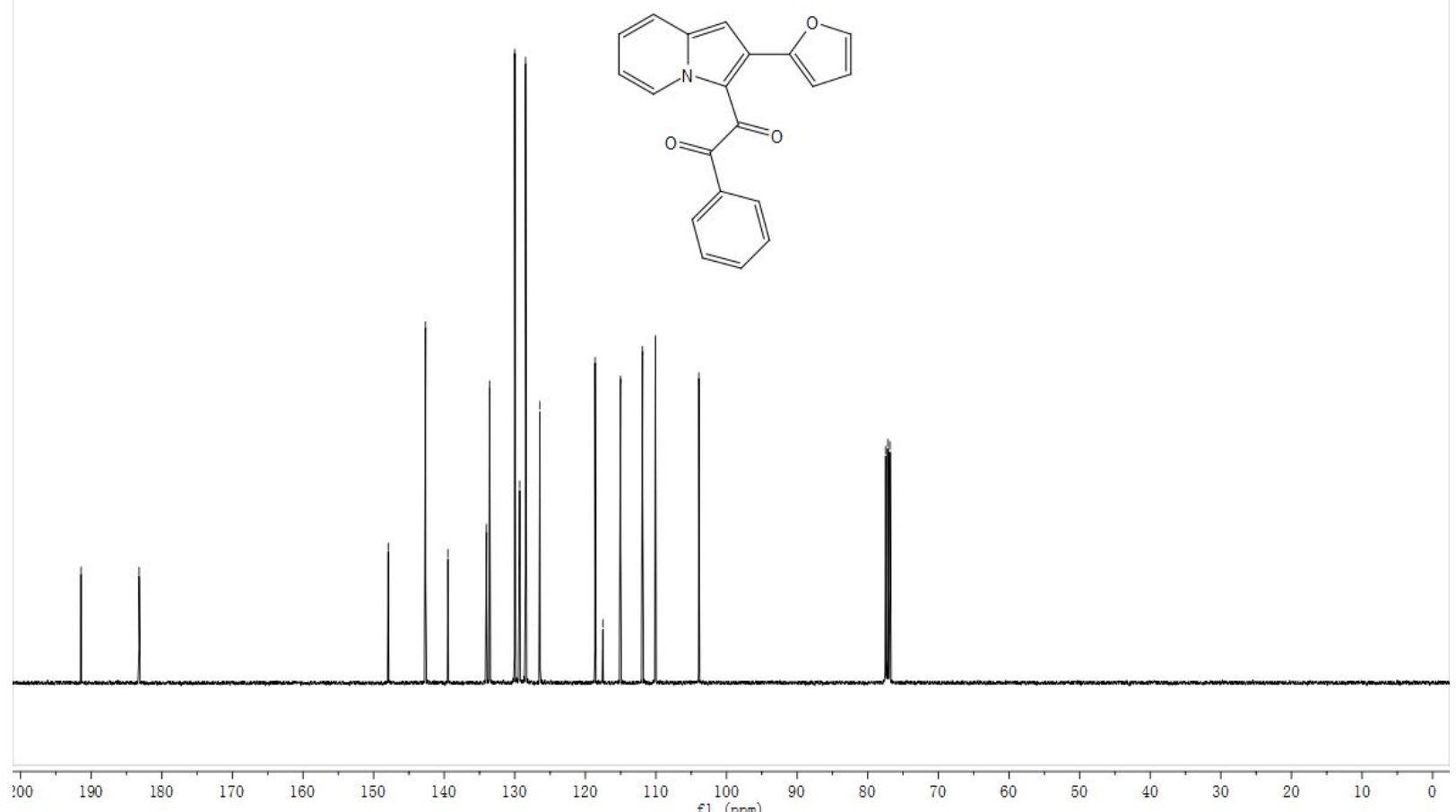
${ }^{1} \mathrm{H}$ NMR (400 MHz, $\mathrm{CDCl}_{3}$ ) spectrum of compound 3s

高望

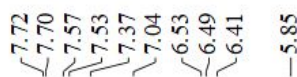
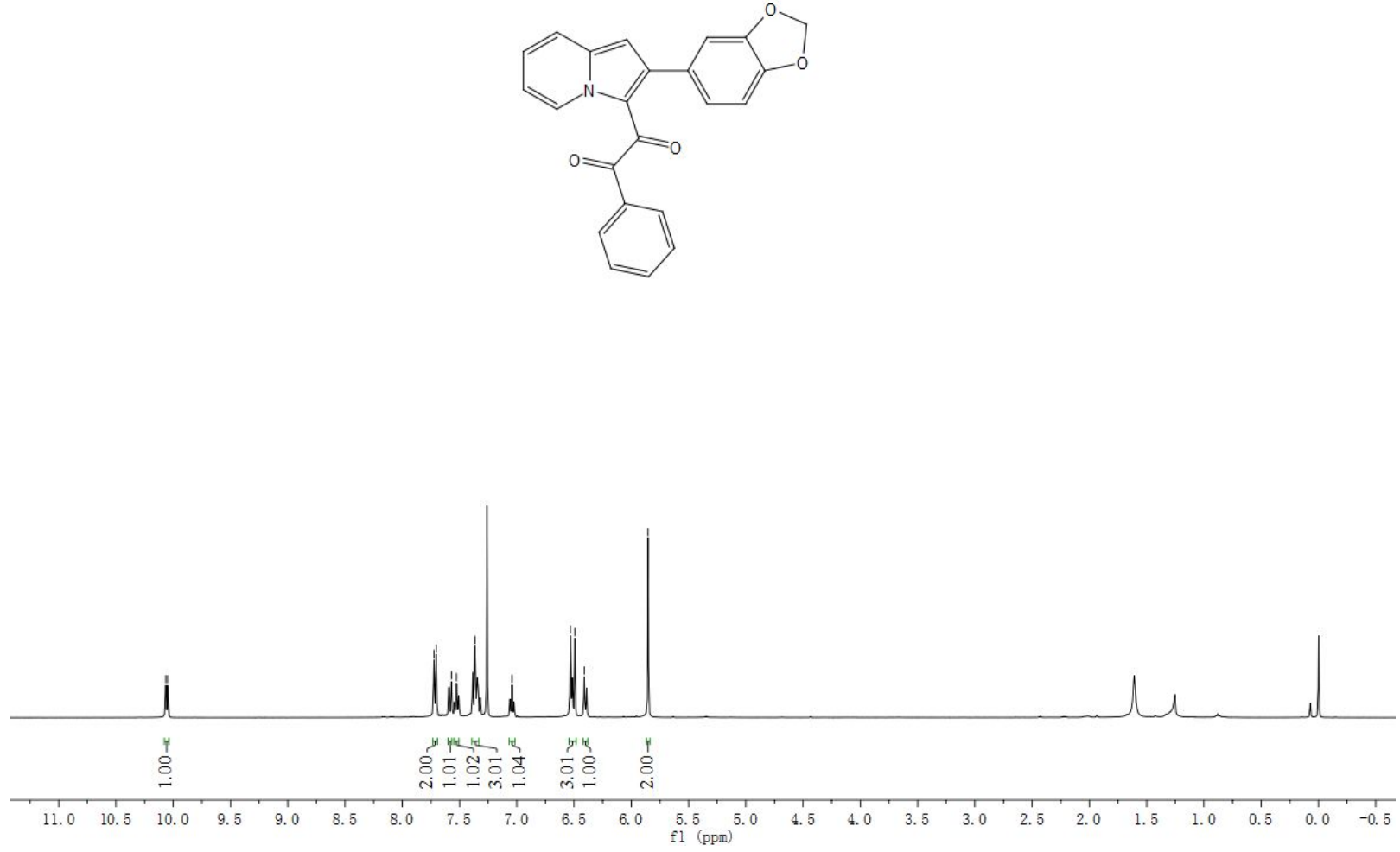

${ }^{13} \mathrm{C} \mathrm{NMR}\left(100 \mathrm{MHz}, \mathrm{CDCl}_{3}\right)$ spectrum of compound $\mathbf{3 s}$

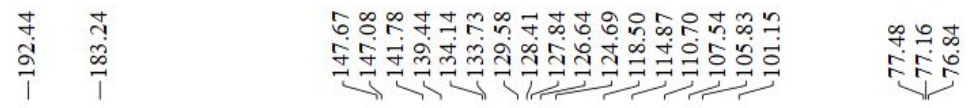
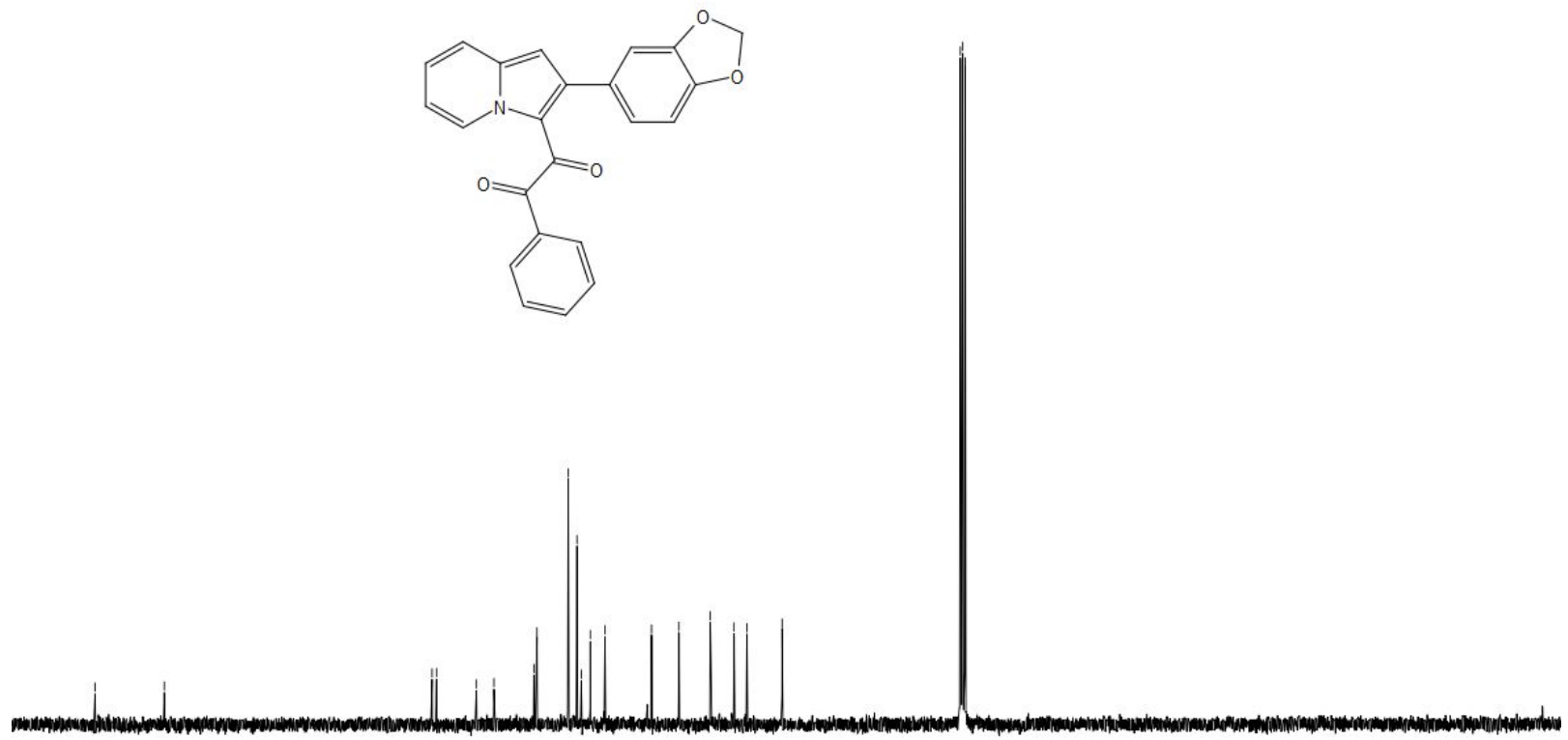

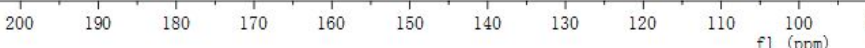


${ }^{1} \mathrm{H}$ NMR (400 MHz, $\mathrm{CDCl}_{3}$ ) spectrum of compound $\mathbf{3 t}$

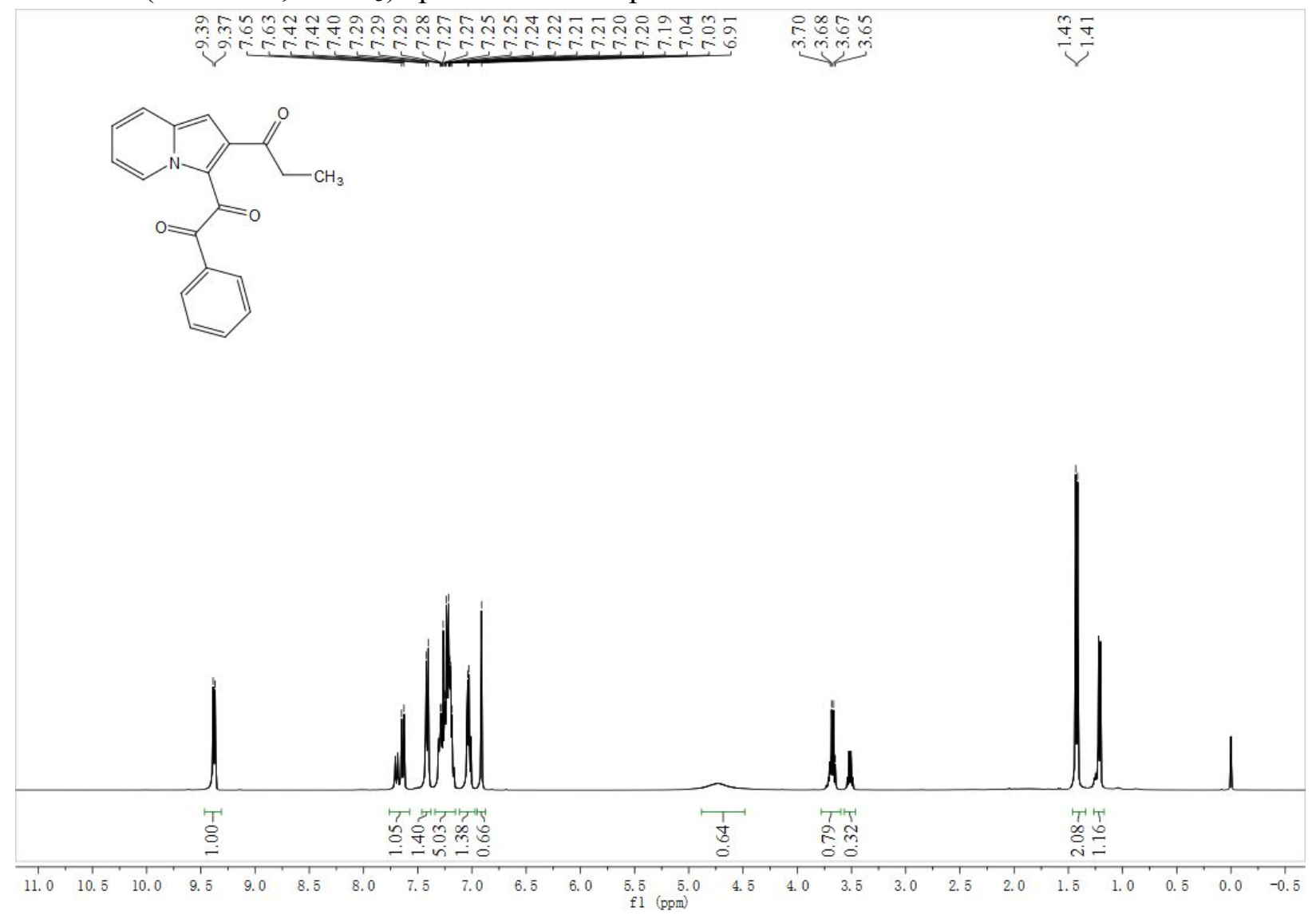

${ }^{13} \mathrm{C}$ NMR $\left(100 \mathrm{MHz}, \mathrm{CDCl}_{3}\right)$ spectrum of compound $\mathbf{3 t}$

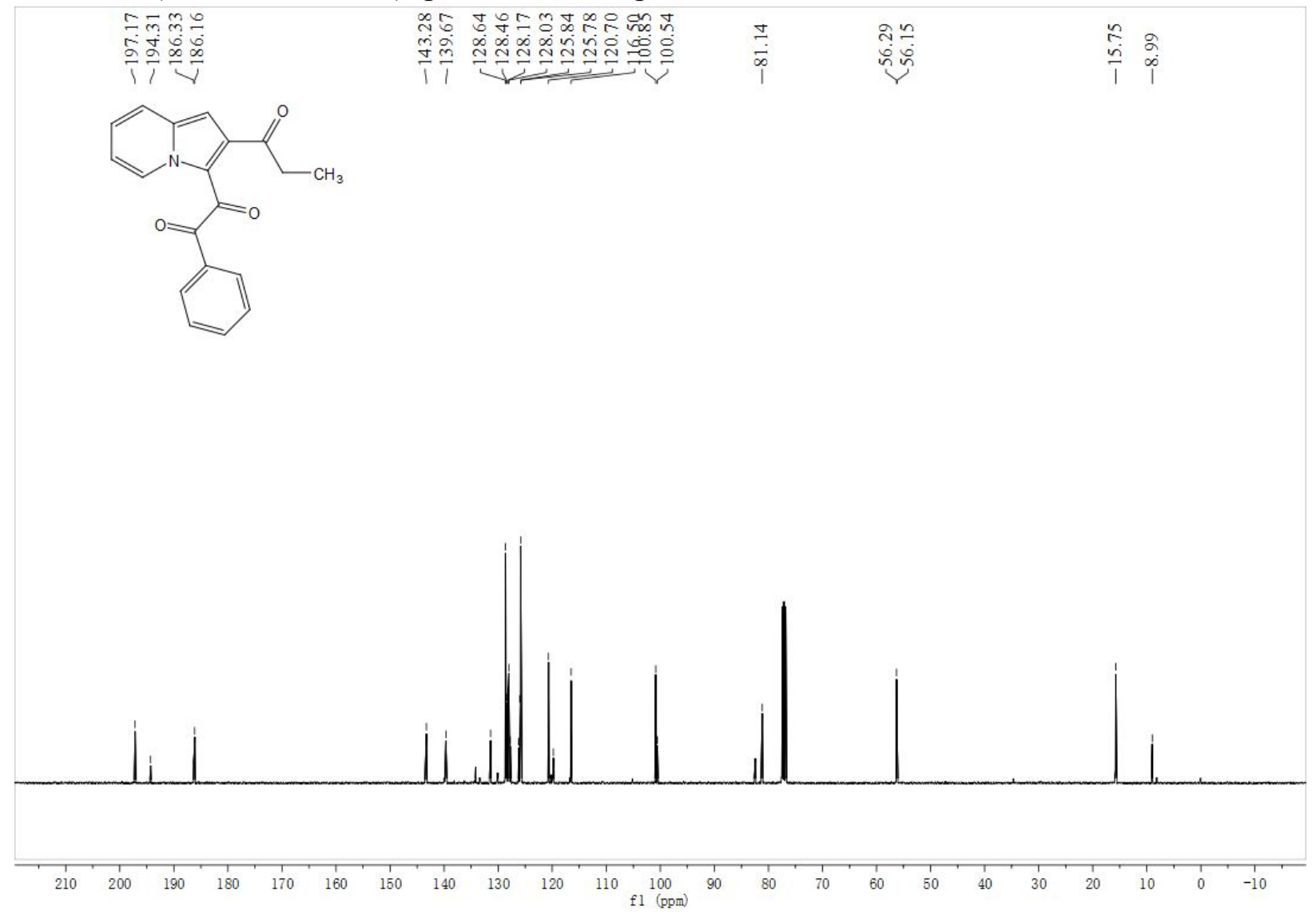

S35 
${ }^{1} \mathrm{H}$ NMR (400 MHz, $\mathrm{CDCl}_{3}$ ) spectrum of compound $\mathbf{3 u}$

等
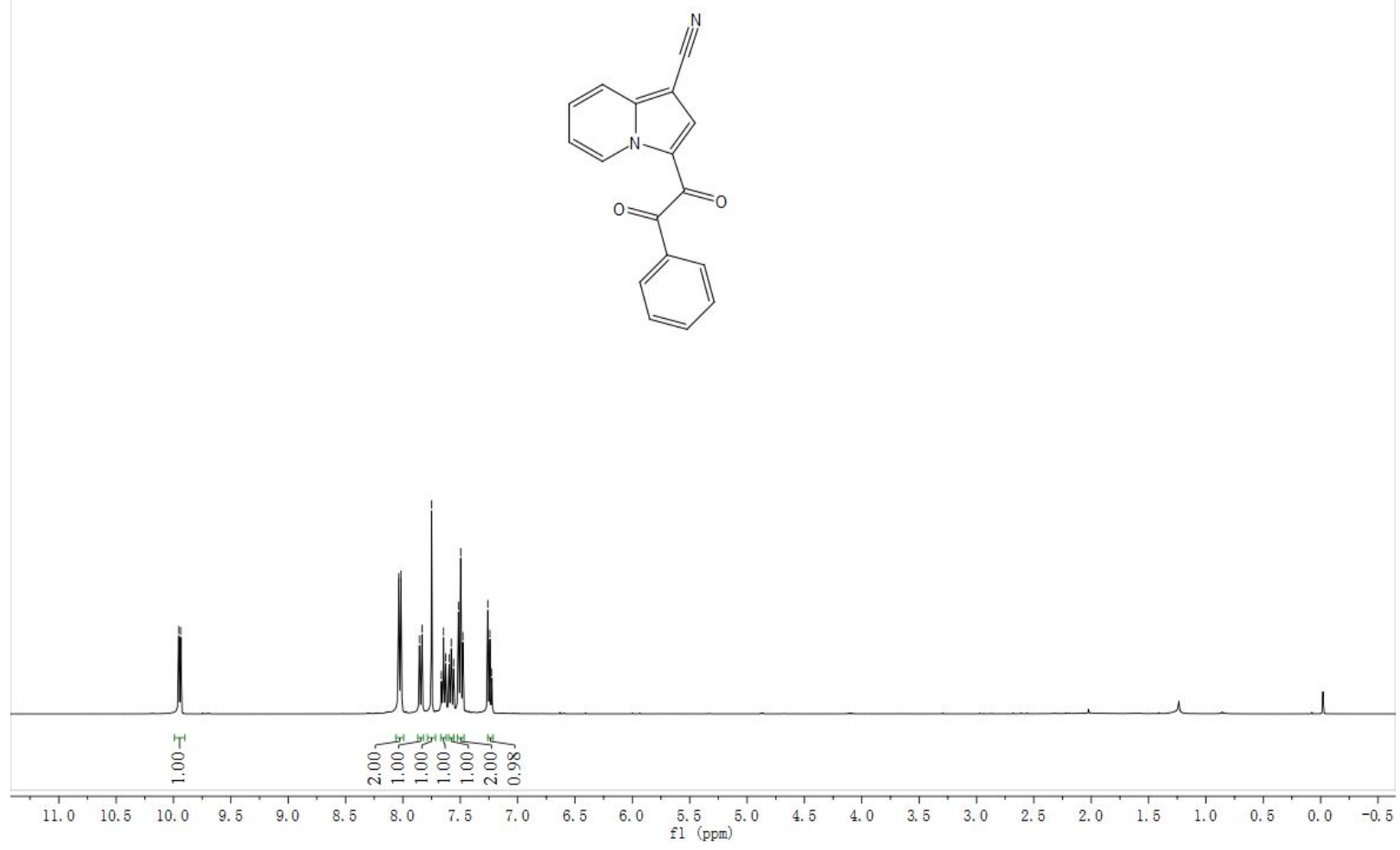

${ }^{13} \mathrm{C}$ NMR $\left(100 \mathrm{MHz}, \mathrm{CDCl}_{3}\right)$ spectrum of compound $\mathbf{3 u}$

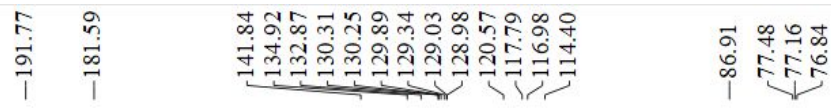<smiles>N#Cc1cc(C(=O)C(=O)c2ccccc2)n2ccccc12</smiles>

$\begin{array}{lllllll}210 & 200 & 190 & 180 & 170 & 160 & 150\end{array}$

$\begin{array}{rrr}140 & 130 & 120\end{array}$ $10 \stackrel{100}{10}$ 
${ }^{1} \mathrm{H}$ NMR (400 MHz, $\mathrm{CDCl}_{3}$ ) spectrum of compound $\mathbf{3 v}$

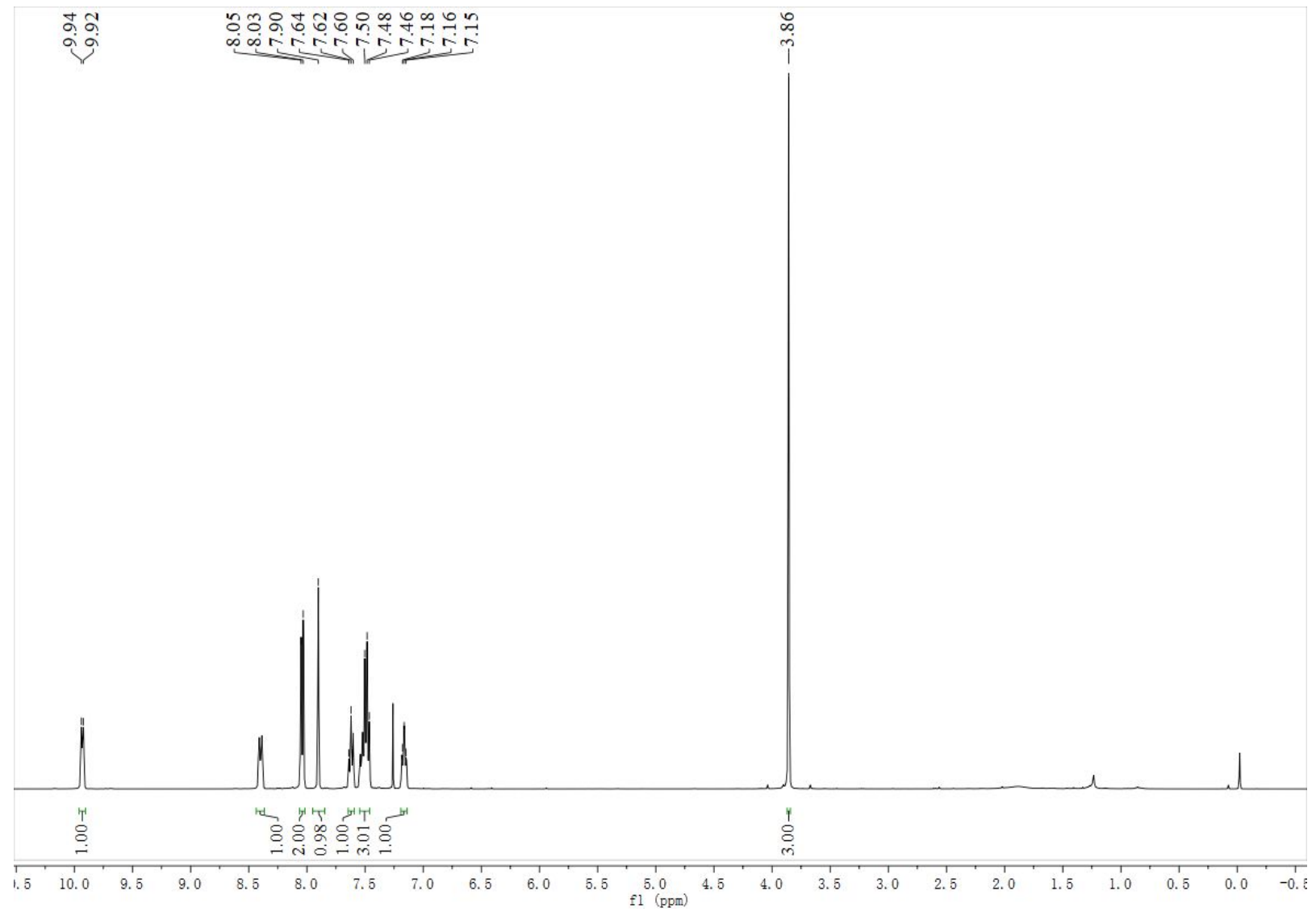

${ }^{13} \mathrm{C}$ NMR $\left(100 \mathrm{MHz}, \mathrm{CDCl}_{3}\right)$ spectrum of compound 3v
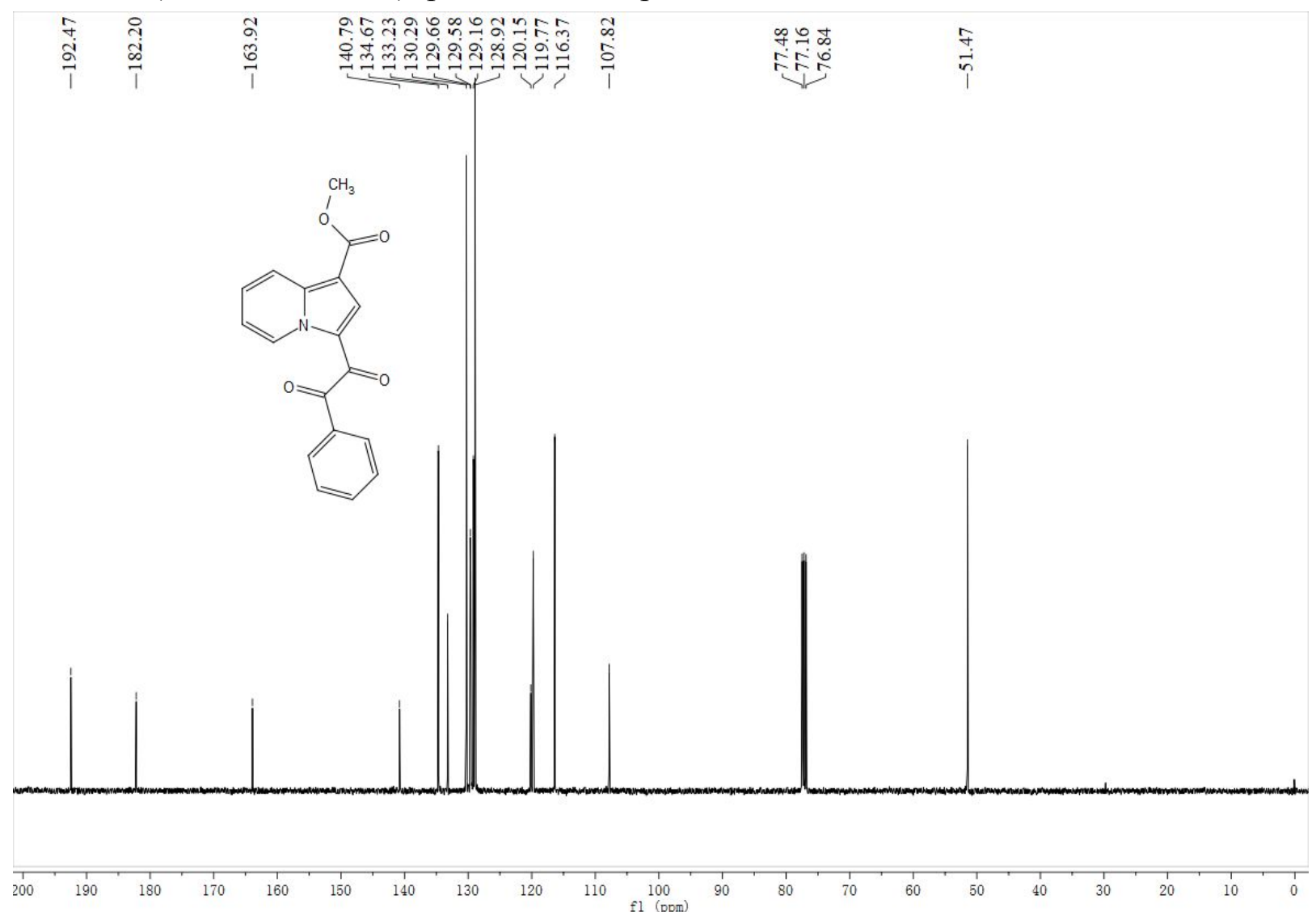
${ }^{1} \mathrm{H}$ NMR (400 MHz, $\left.\mathrm{CDCl}_{3}\right)$ spectrum of compound $4 \mathbf{a}$

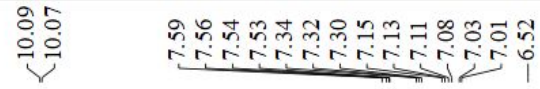
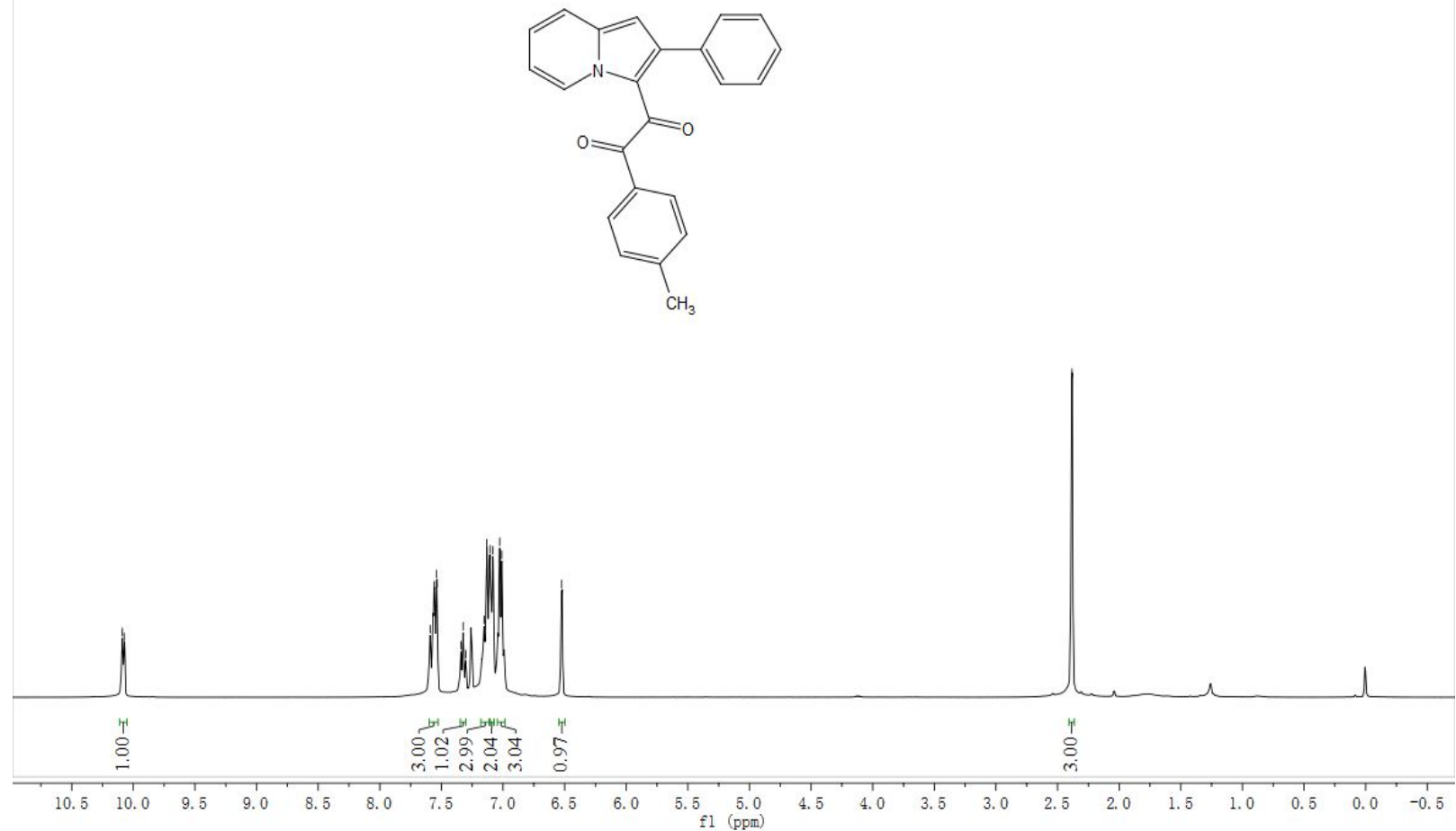

${ }^{13} \mathrm{C}$ NMR $\left(100 \mathrm{MHz}, \mathrm{CDCl}_{3}\right)$ spectrum of compound $4 \mathbf{a}$

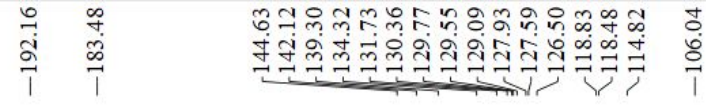
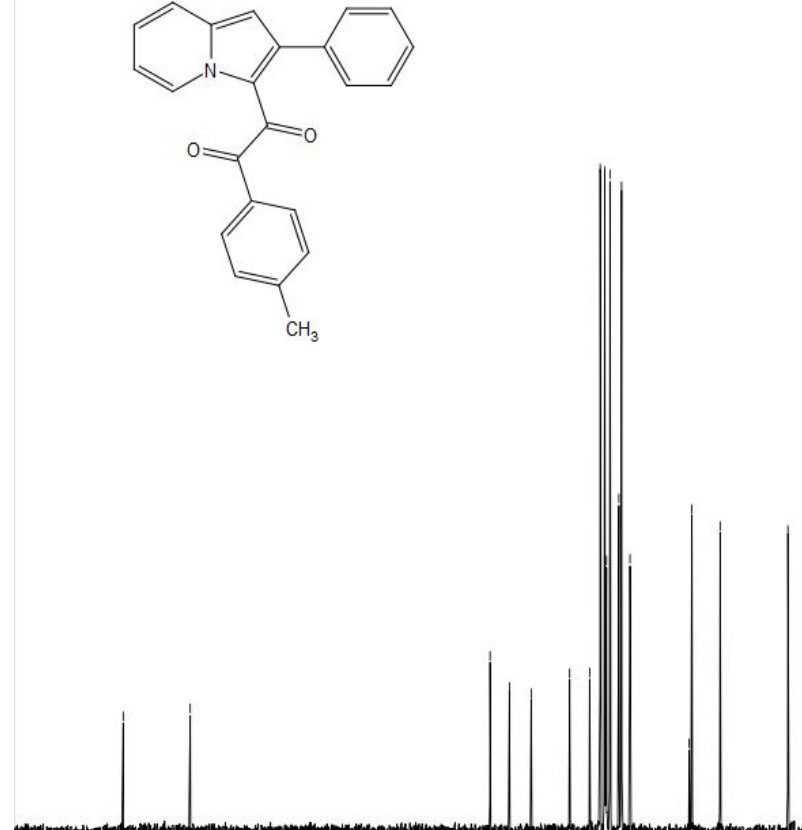

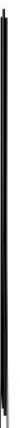
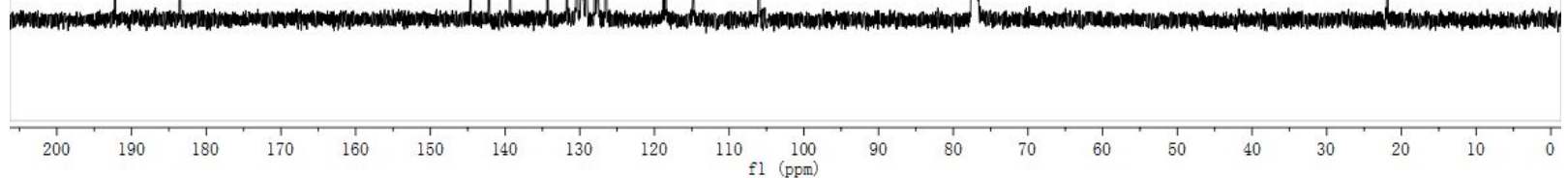
${ }^{1} \mathrm{H}$ NMR (400 MHz, $\mathrm{CDCl}_{3}$ ) spectrum of compound $\mathbf{4 b}$

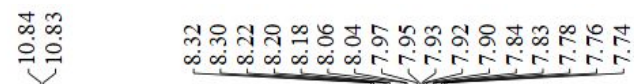

$\stackrel{\text { co }}{\text { i }}$
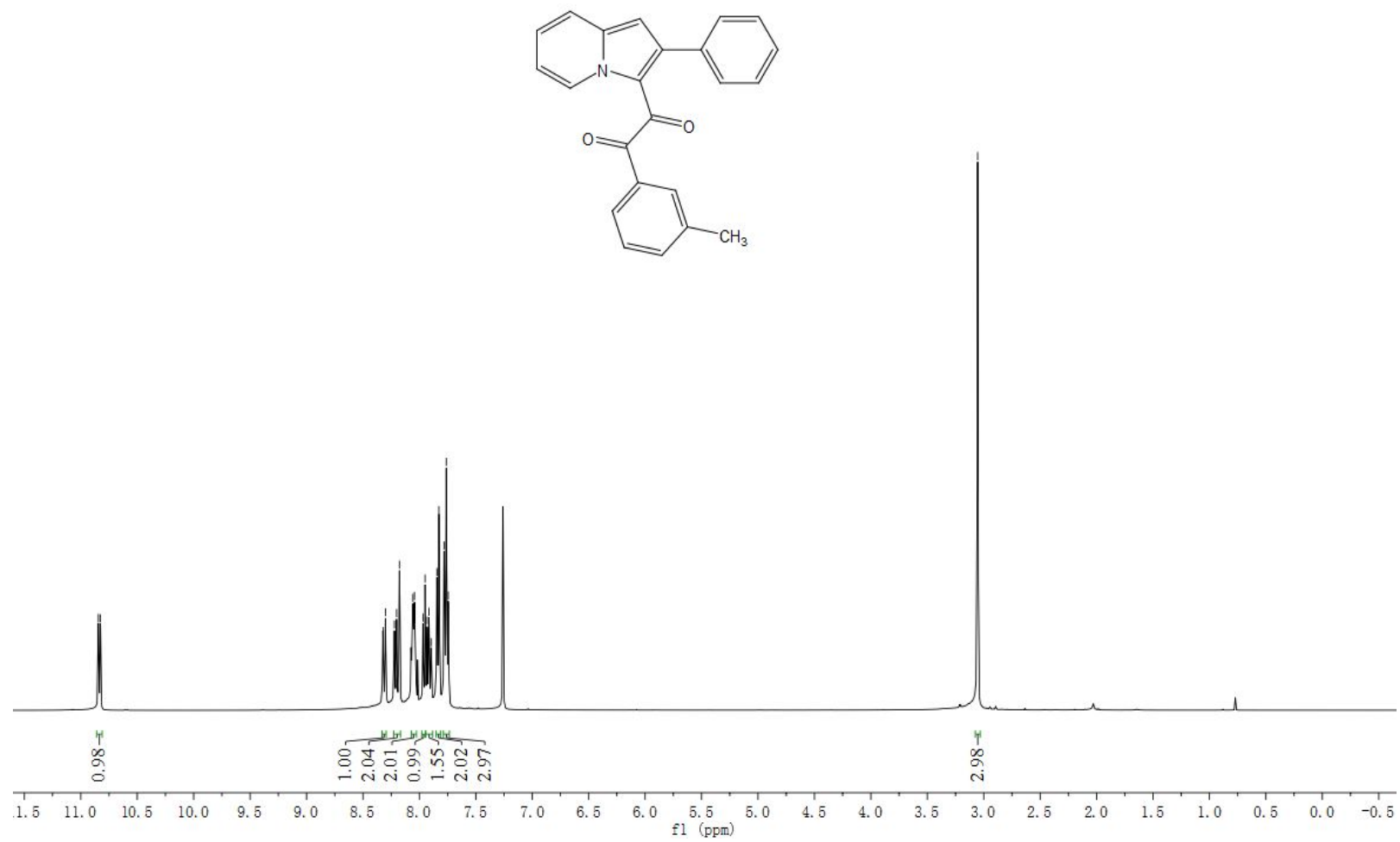

${ }^{13} \mathrm{C} \mathrm{NMR}\left(100 \mathrm{MHz}, \mathrm{CDCl}_{3}\right)$ spectrum of compound $4 \mathbf{b}$

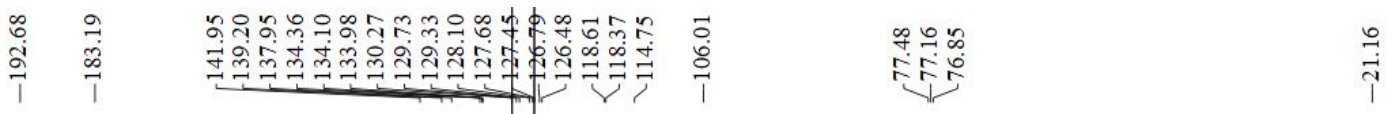

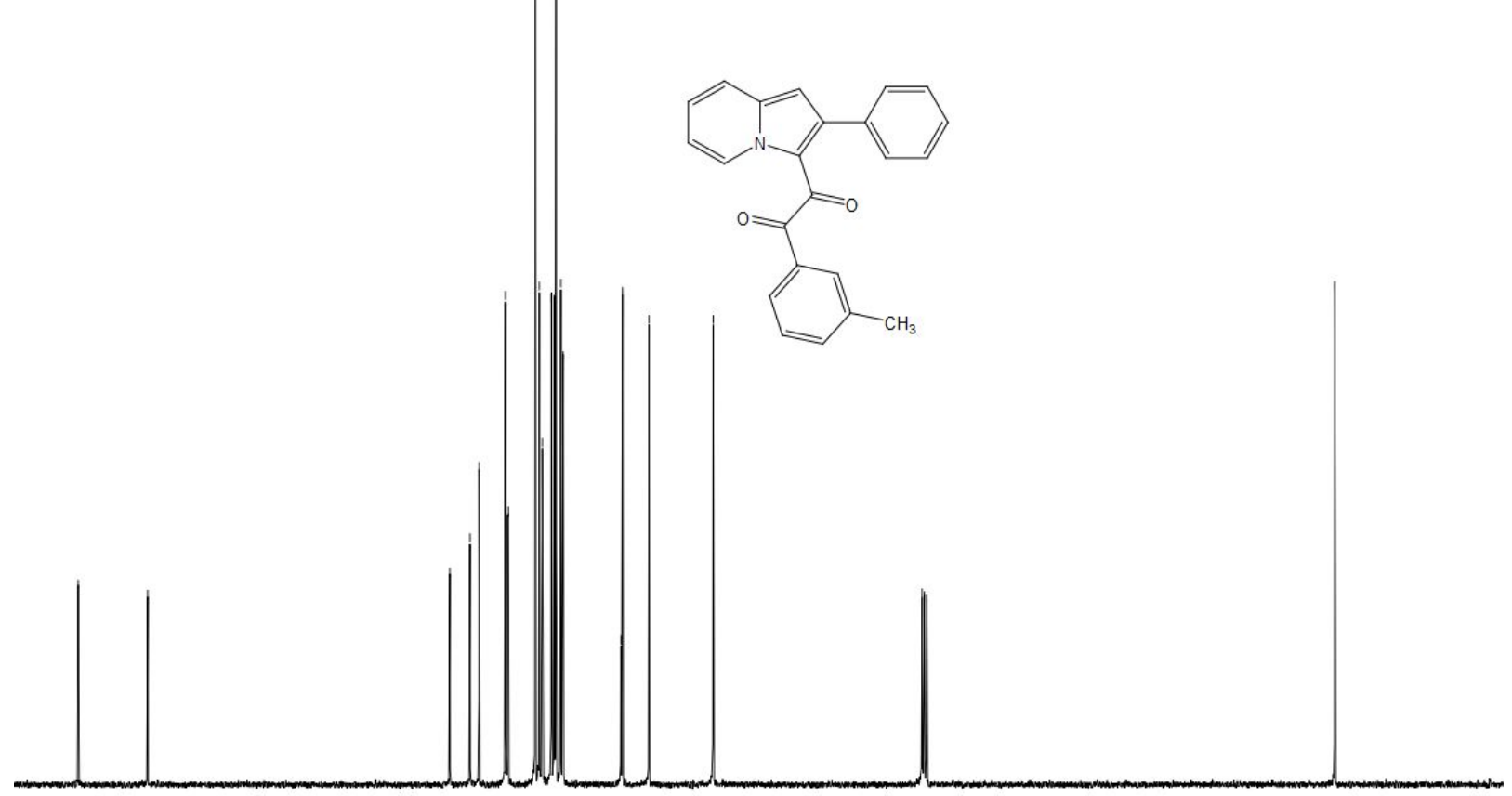

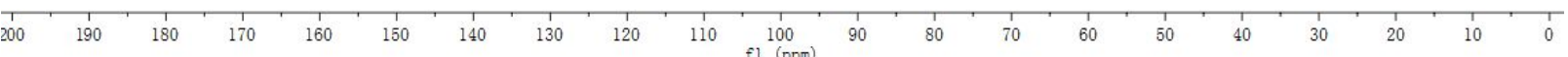


${ }^{1} \mathrm{H}$ NMR (400 MHz, $\mathrm{CDCl}_{3}$ ) spectrum of compound $\mathbf{4 c}$

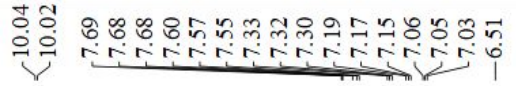
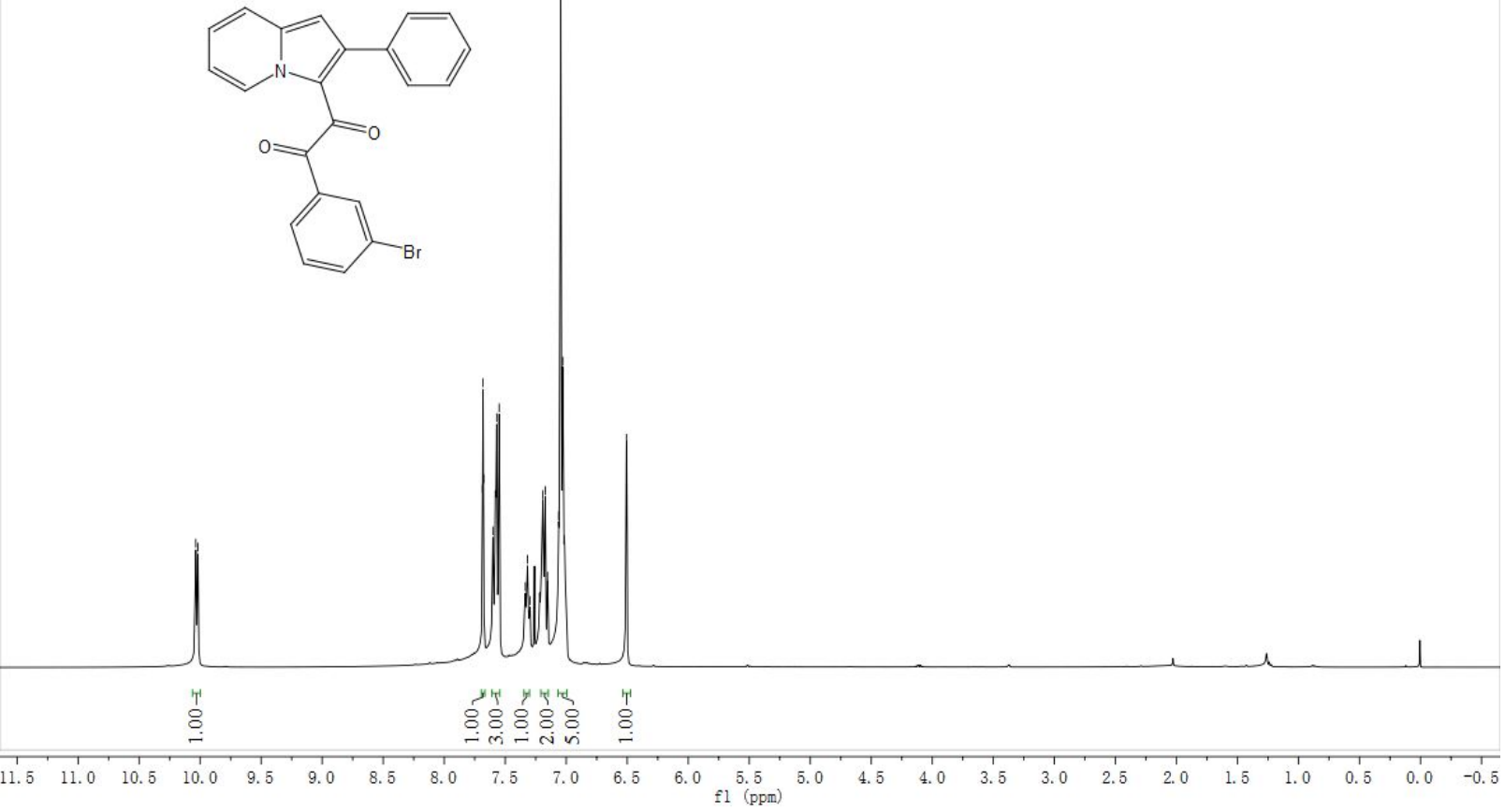

${ }^{13} \mathrm{C}$ NMR $\left(100 \mathrm{MHz}, \mathrm{CDCl}_{3}\right)$ spectrum of compound $4 \mathbf{c}$
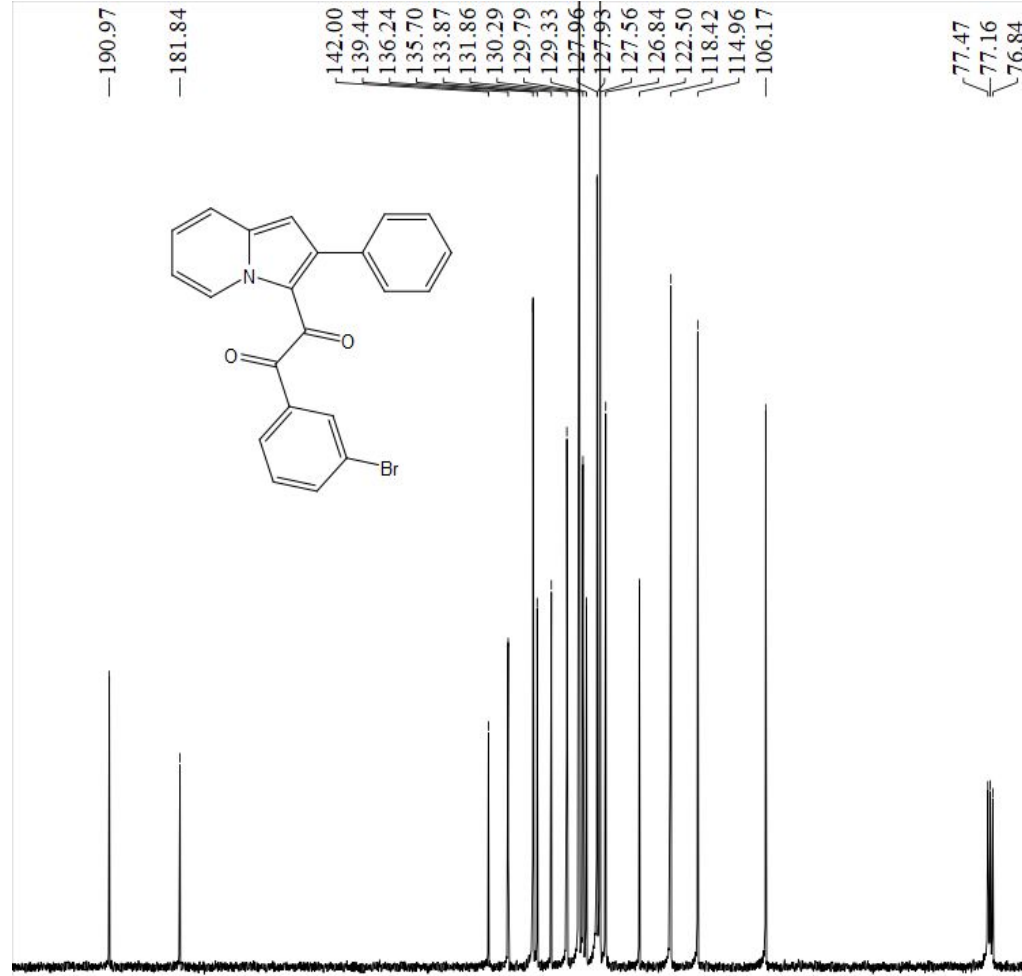

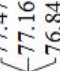

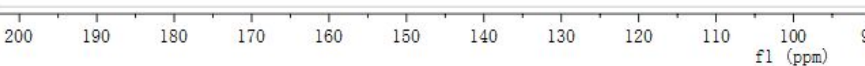


${ }^{1} \mathrm{H}$ NMR (400 MHz, $\mathrm{CDCl}_{3}$ ) spectrum of compound $\mathbf{4 d}$

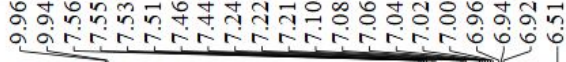
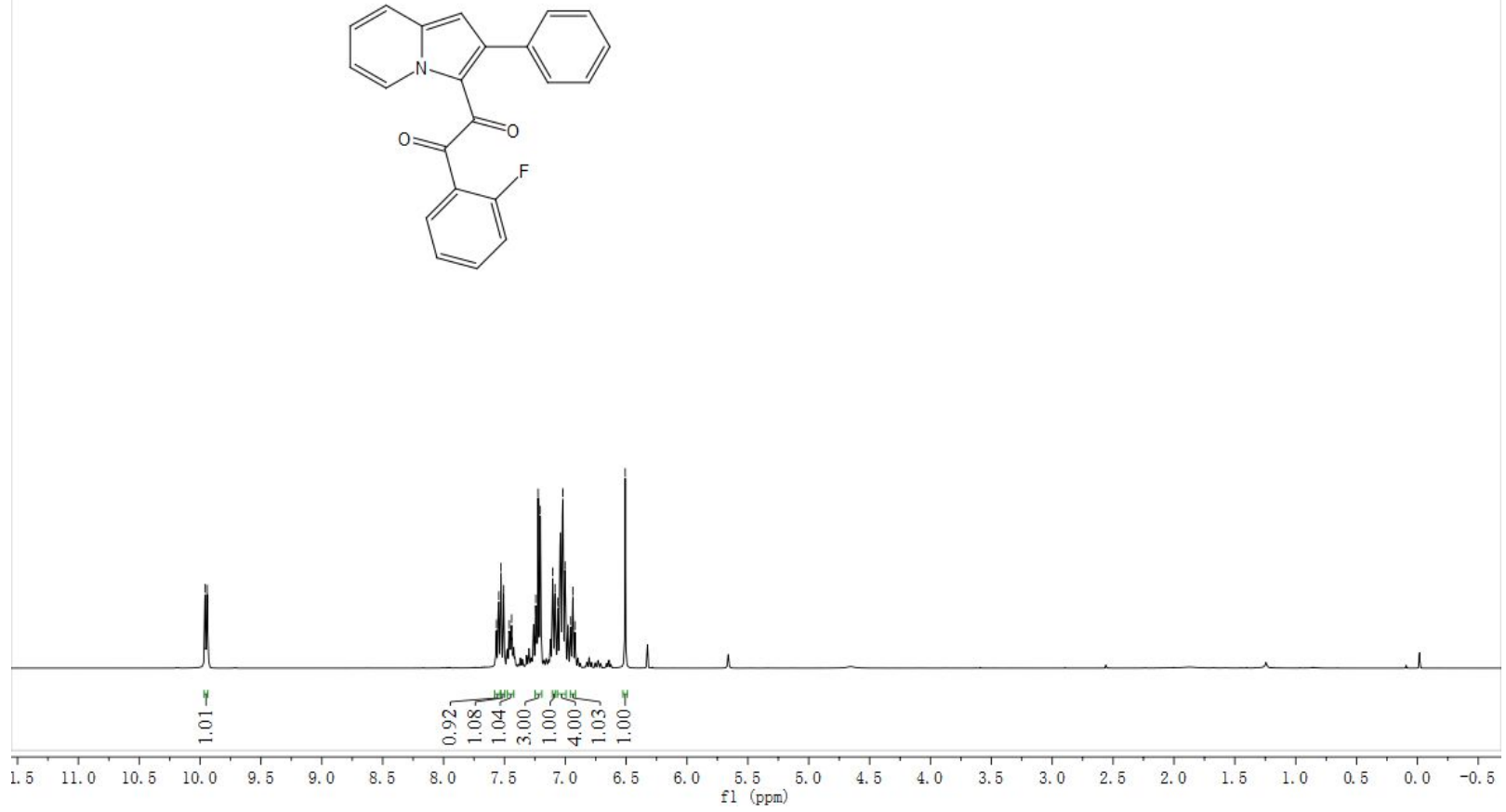

${ }^{13} \mathrm{C}$ NMR $\left(100 \mathrm{MHz}, \mathrm{CDCl}_{3}\right)$ spectrum of compound $4 \mathbf{d}$

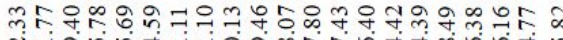

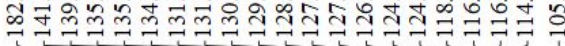

里유뭉

됴요
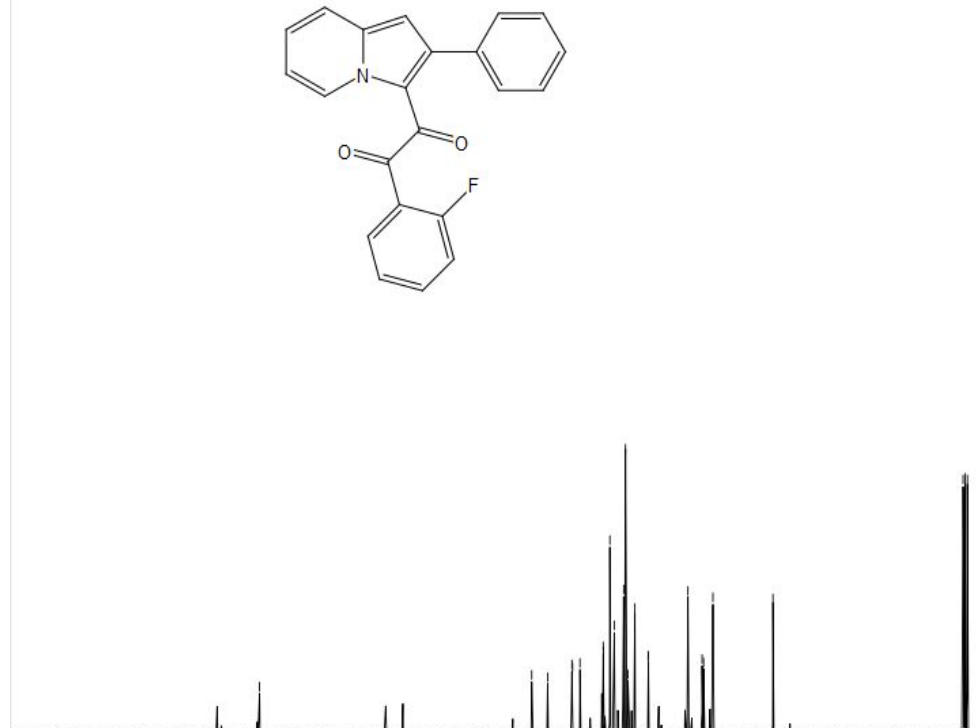

$\begin{array}{llllllllllll}210 & 200 & 190 & 180 & 170 & 160 & 150 & 140 & 130 & 120 & 110 & 100 \\ & & & & & & & & \end{array}$ 
${ }^{1} \mathrm{H}$ NMR (400 MHz, $\mathrm{CDCl}_{3}$ ) spectrum of compound $\mathbf{4 e}$

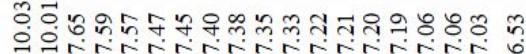

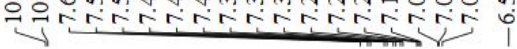
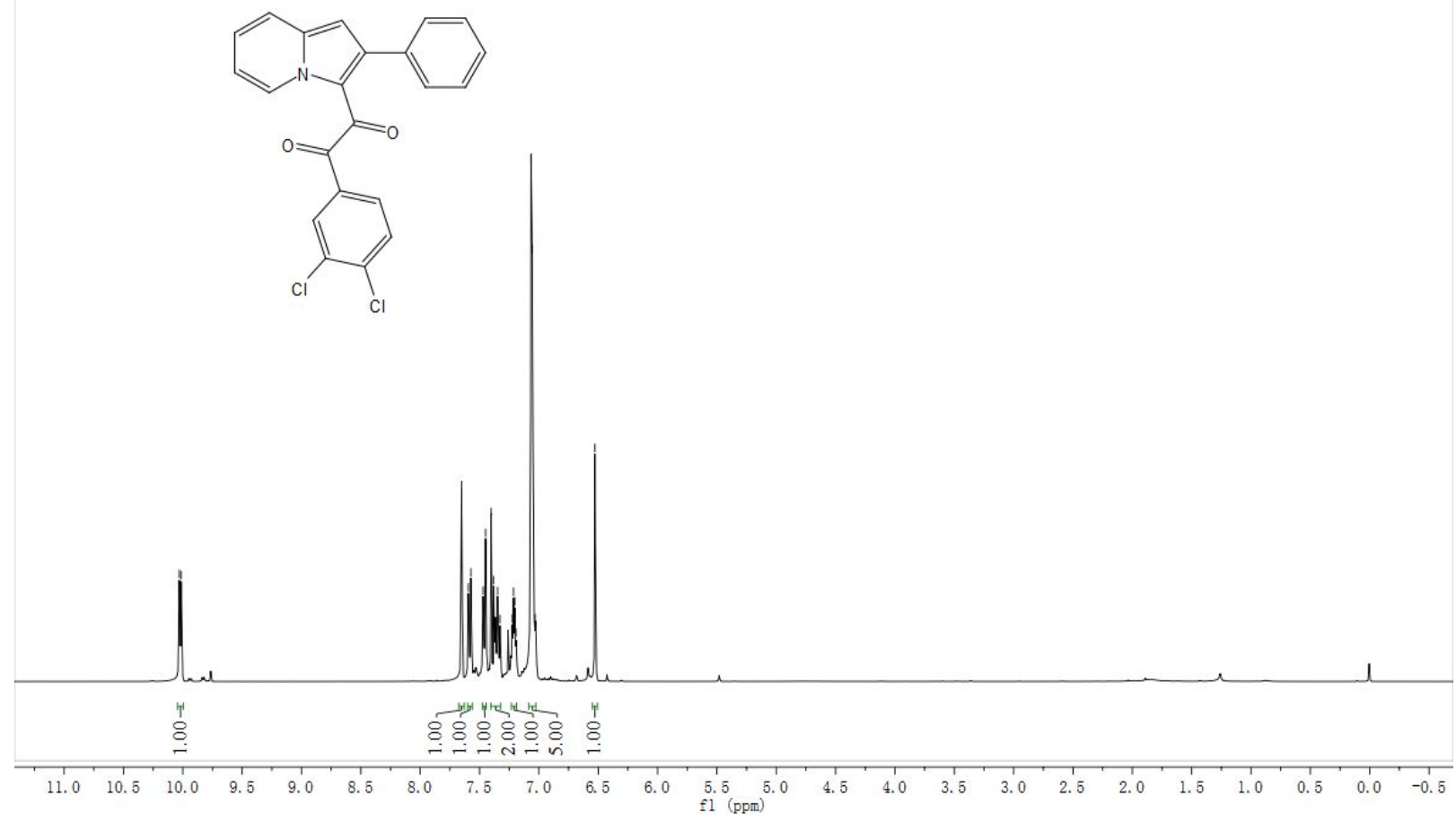

${ }^{13} \mathrm{C} \mathrm{NMR}\left(100 \mathrm{MHz}, \mathrm{CDCl}_{3}\right)$ spectrum of compound $4 \mathbf{e}$

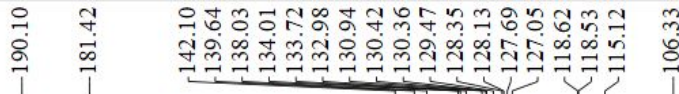

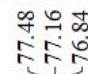
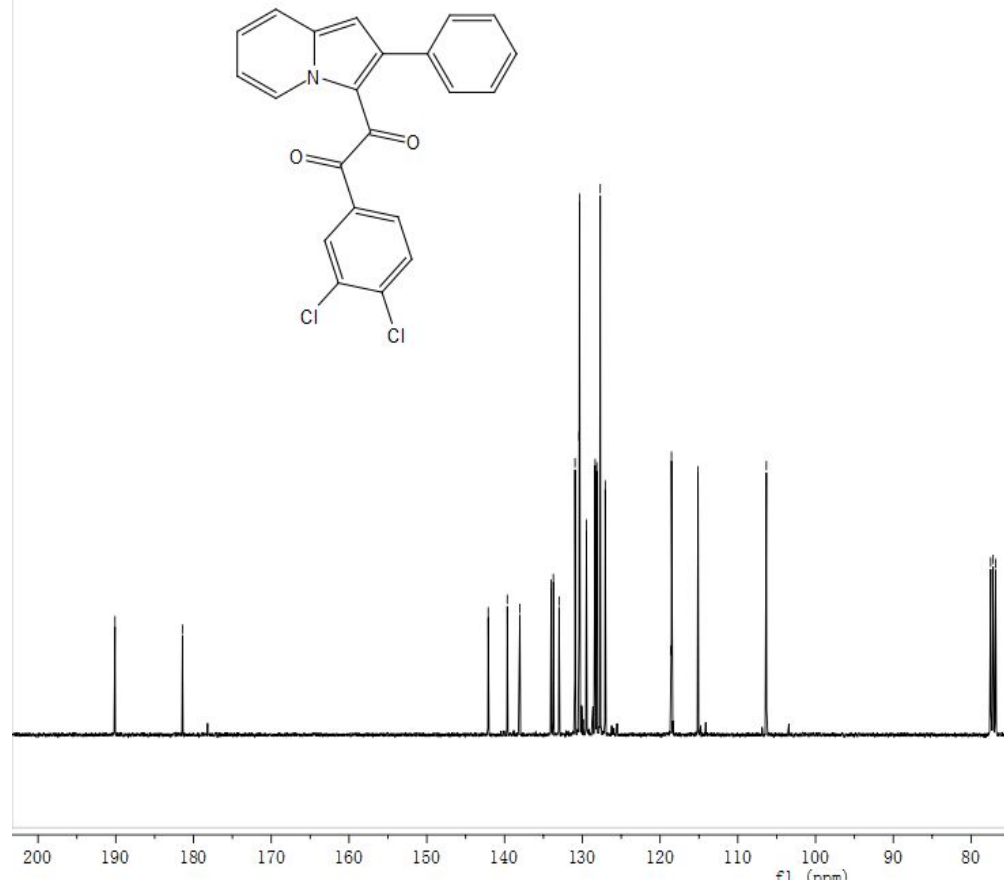
${ }^{1} \mathrm{H}$ NMR (400 MHz, $\left.\mathrm{CDCl}_{3}\right)$ spectrum of compound $\mathbf{4 f}$

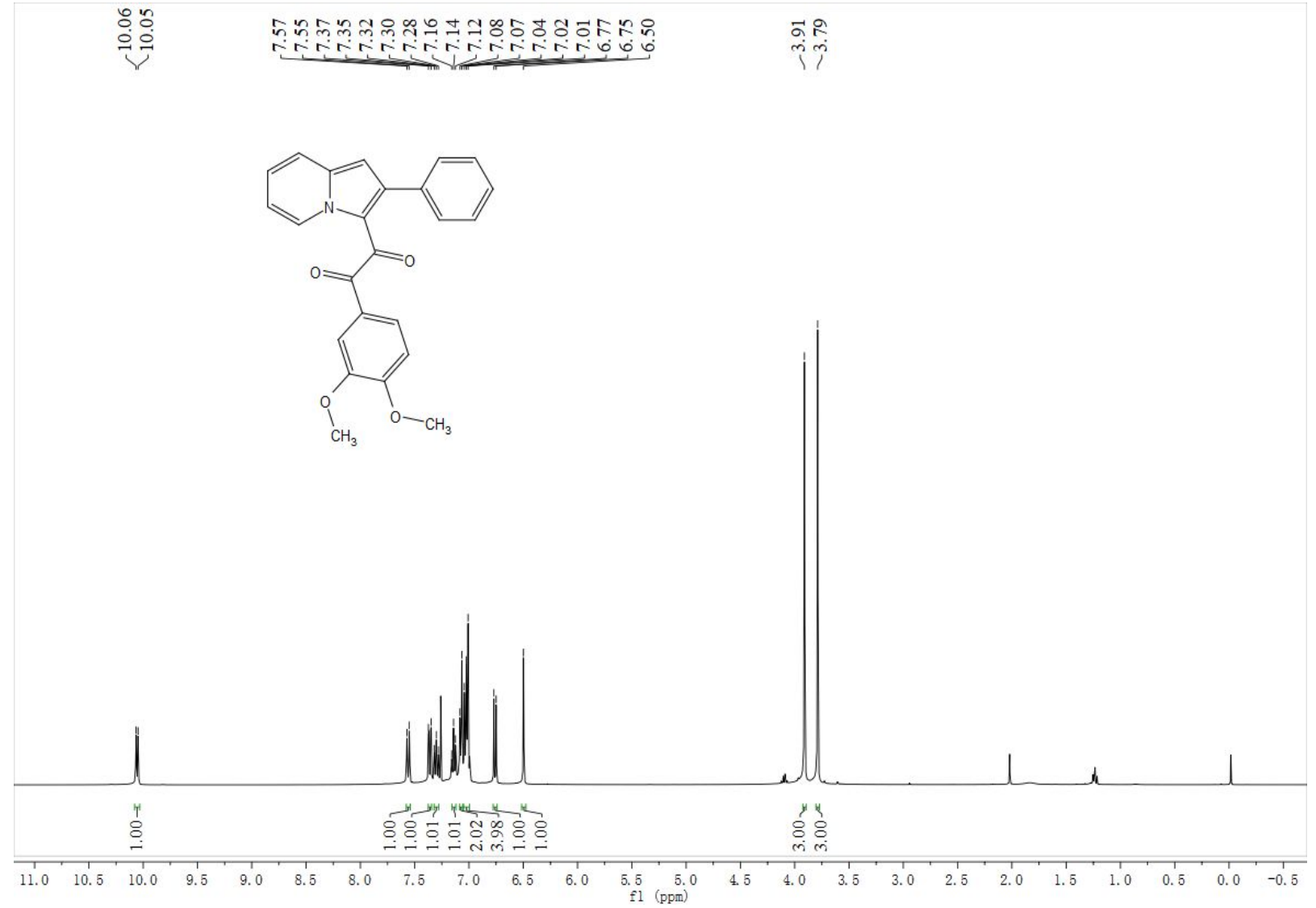

${ }^{13} \mathrm{C}$ NMR $\left(100 \mathrm{MHz}, \mathrm{CDCl}_{3}\right)$ spectrum of compound $\mathbf{4 f}$

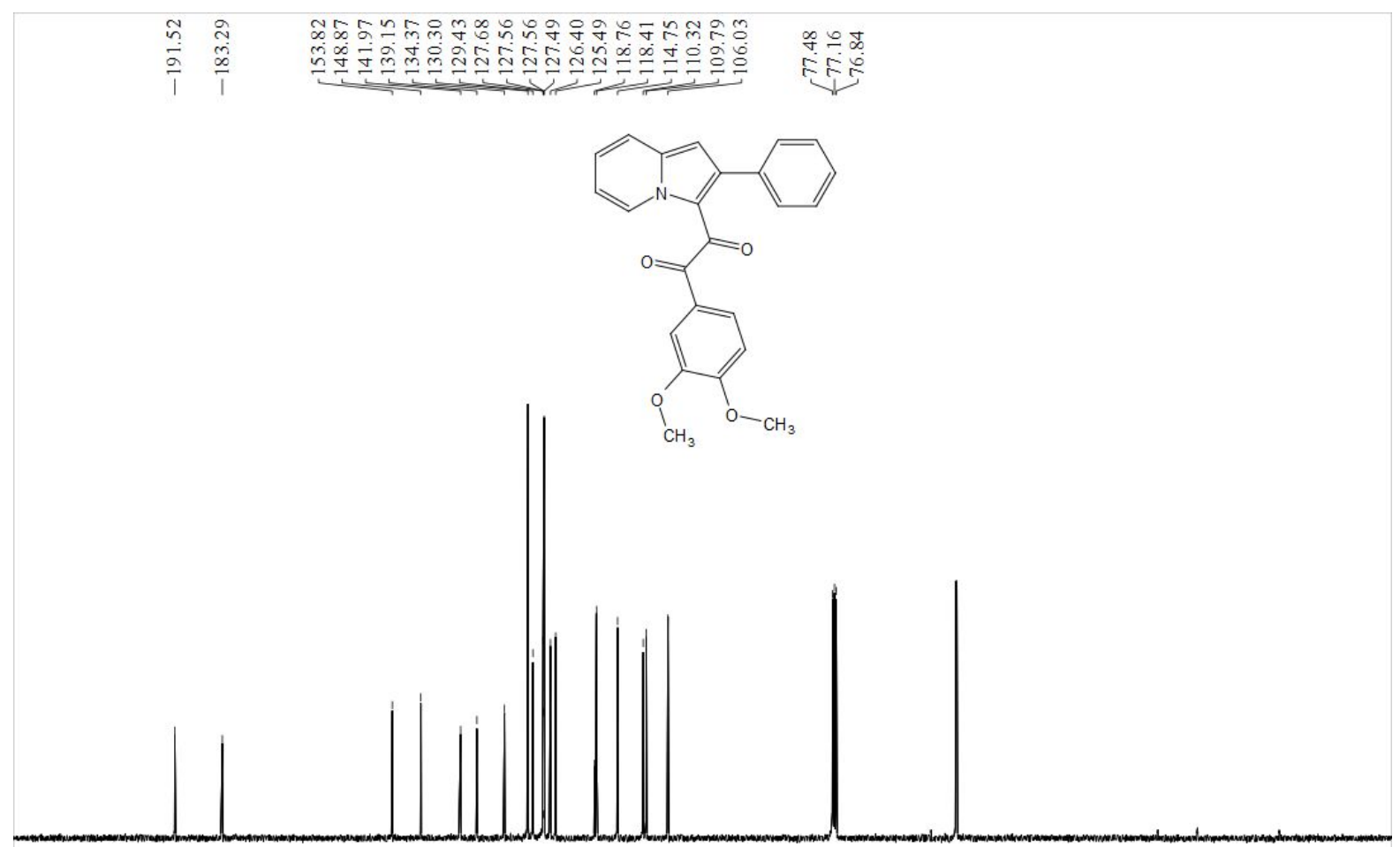

$\begin{array}{llllllllllllllllllllllllllllllllllll}1 & 1 & 200 & 190 & 180 & 170 & 160 & 150 & 140 & 130 & 120 & 110 & 100 & 90 & 80 & 70 & 60 & 50 & 40 & 30 & 20 & 10 & 0 & -10\end{array}$ 
${ }^{1} \mathrm{H}$ NMR (400 MHz, $\mathrm{CDCl}_{3}$ ) spectrum of compound $\mathbf{4 g}$

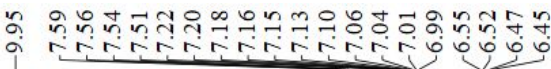
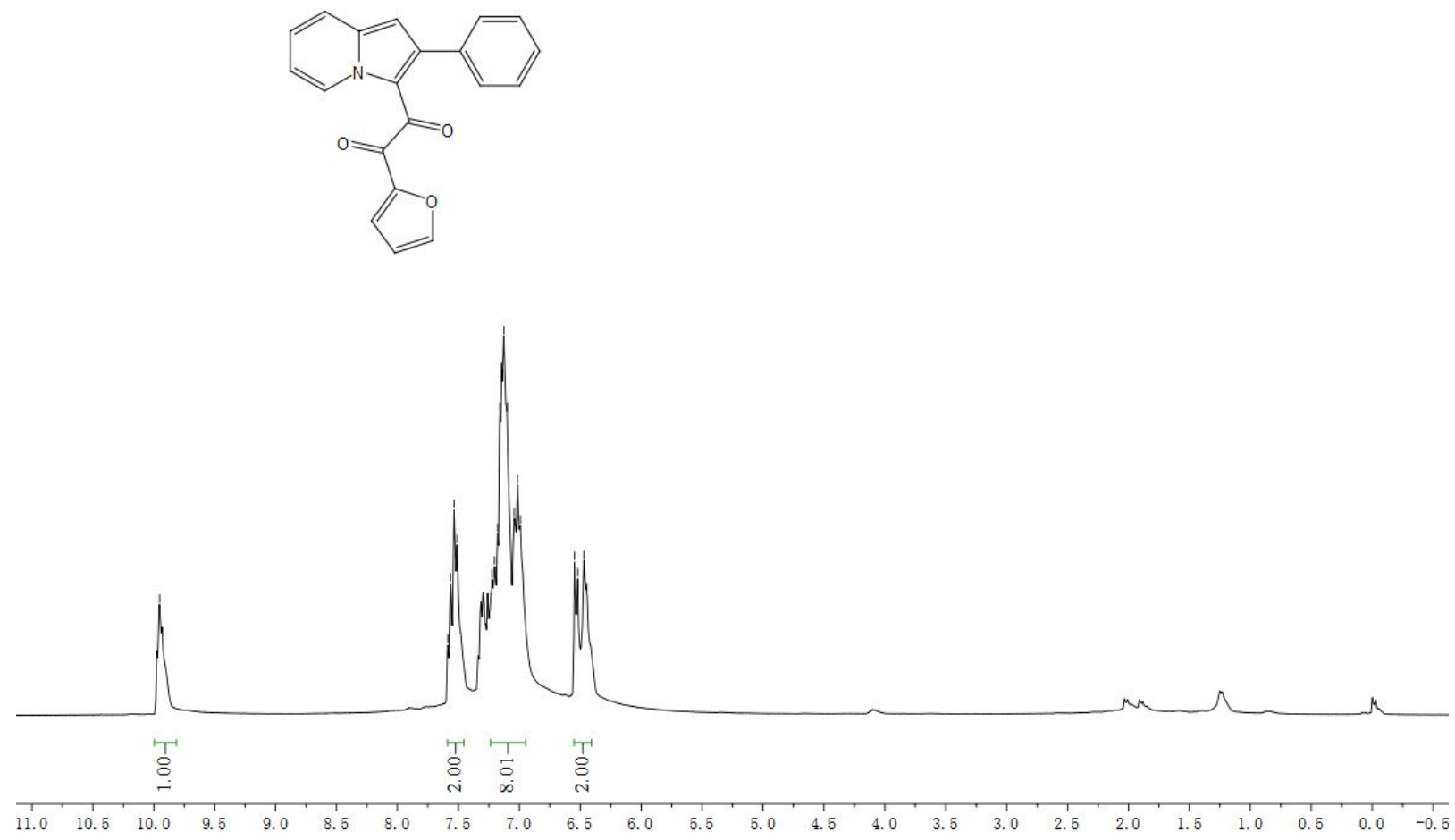

${ }^{13} \mathrm{C}$ NMR $\left(100 \mathrm{MHz}, \mathrm{CDCl}_{3}\right)$ spectrum of compound $\mathbf{4 g}$
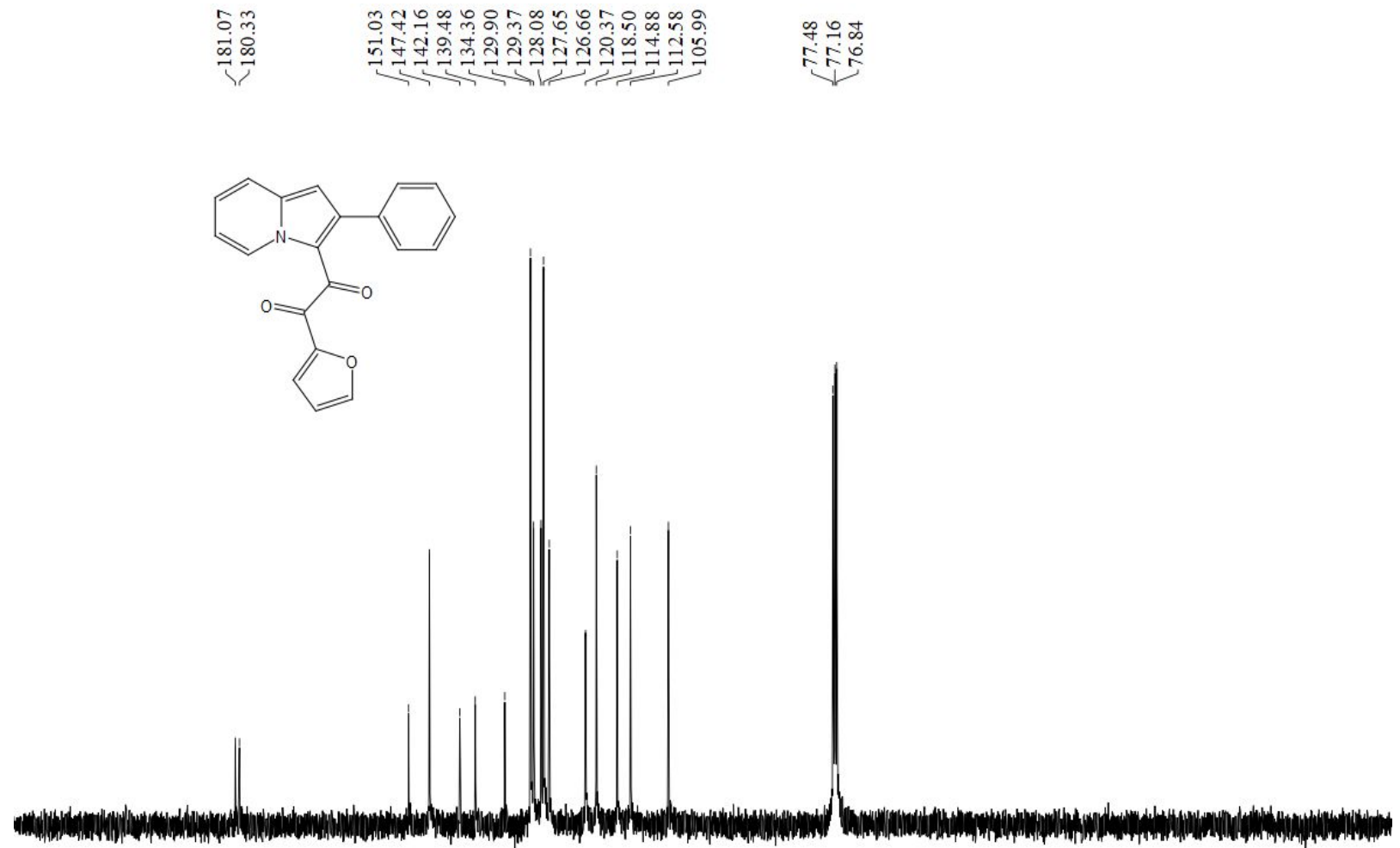

-15
50
50
50

$\begin{array}{lllllllllll}210 & 200 & 190 & 180 & 170 & 160 & 150 & 140 & 130 & 120 & 110\end{array}$ 
${ }^{1} \mathrm{H}$ NMR (400 MHz, $\mathrm{CDCl}_{3}$ ) spectrum of compound $\mathbf{4 h}$

落
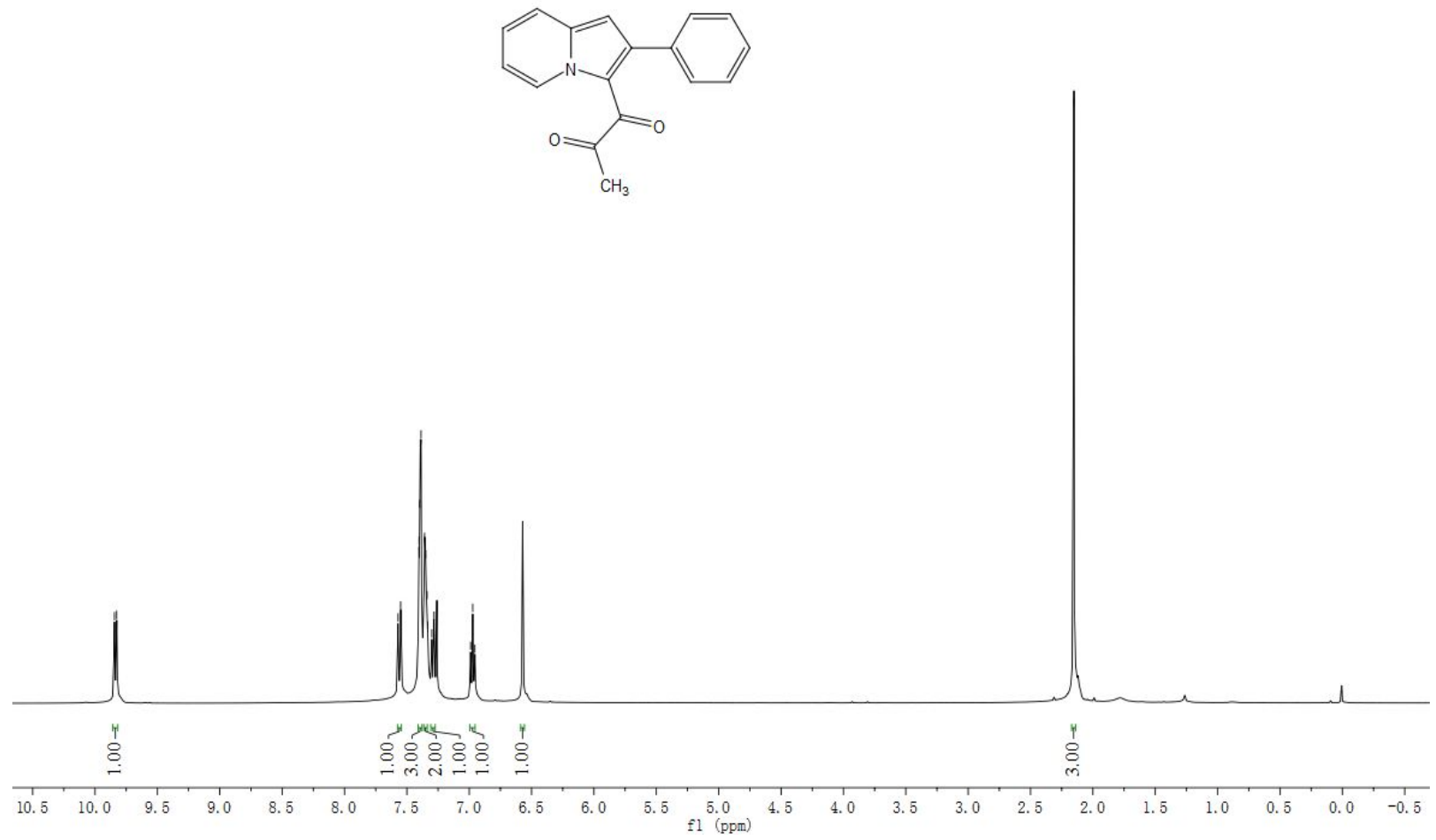

${ }^{13} \mathrm{C}$ NMR $\left(100 \mathrm{MHz}, \mathrm{CDCl}_{3}\right)$ spectrum of compound $\mathbf{4 h}$

m $\stackrel{m}{m}$

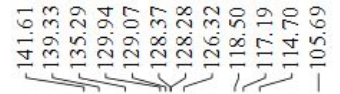

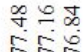

ह5

$\underset{\substack{\text { ind } \\ \text { i }}}{ }$

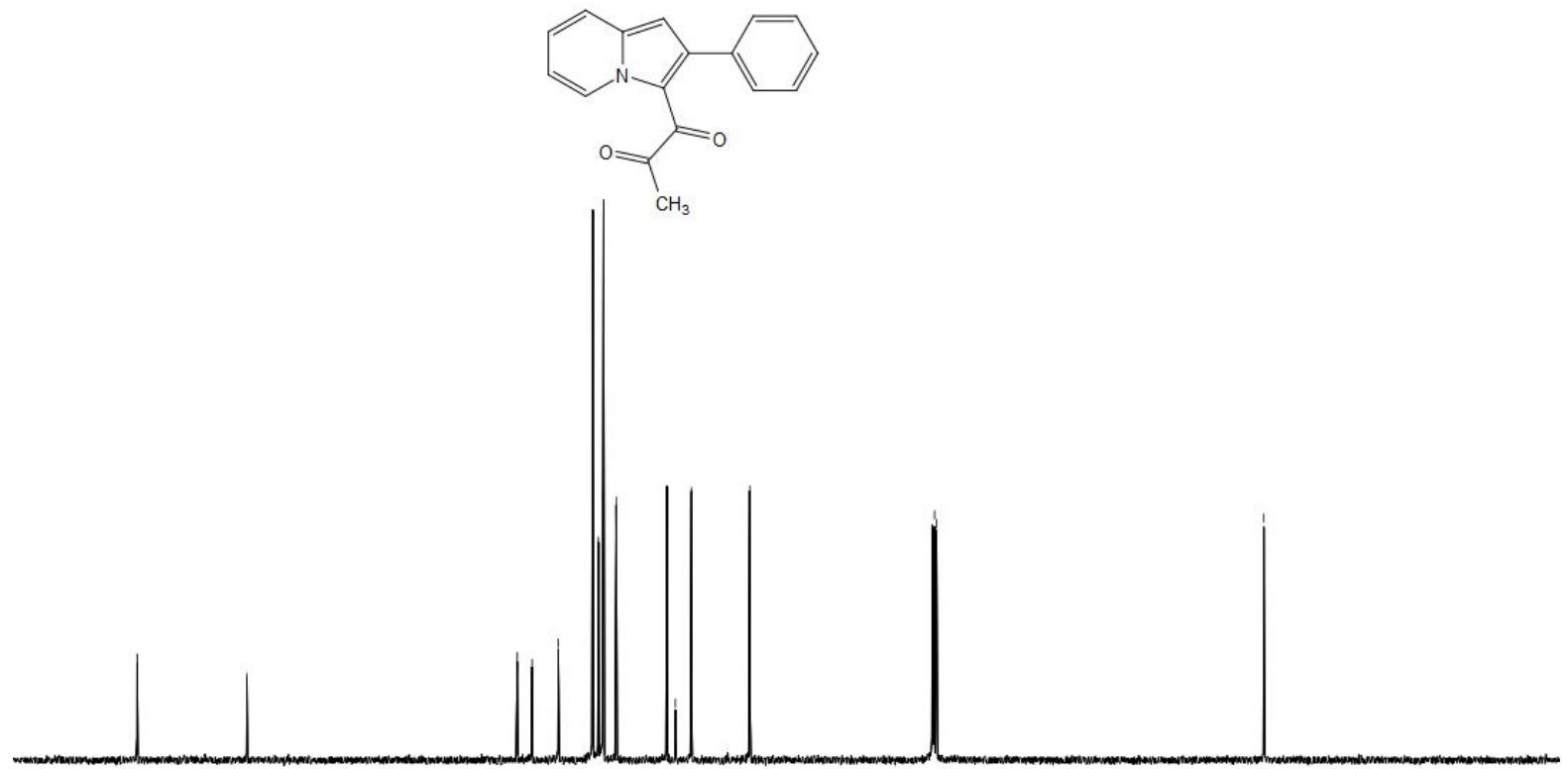

$\begin{array}{llllllllllll}210 & 200 & 190 & 180 & 170 & 160 & 150 & 140 & 130 & 120 & 110 & 100 \\ \mathrm{fl} & (\mathrm{pgm})\end{array}$ 


\section{References}

1. Li, B.; Chen, Z.; Cao, H.; Zhao, H. Org. Lett. 2018, 20, 3291-3295.

2. Gundersena, L. L.; Charnockb, C.; Negussie, A. H.; Rise, F.; Teklua, S. Eur. J. Pha. Science. 2007, 30, 26-35.

3. Batchu, H.; Batra, S. Eur. J. Org. Chem. 2012, 15, 2935-2944. 